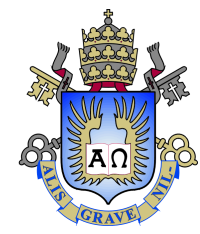

Giovanna Luisa Coelho Leal

A realização de alguns subgrupos discretos do grupo Spin na álgebra de Clifford

Dissertação apresentada como requisito parcial para obtenção do grau de Mestre pelo Programa de Pós-graduação em Matemática, do Departamento de Matemática da PUC-Rio.

Orientador: Prof. Nicolau Corção Saldanha 


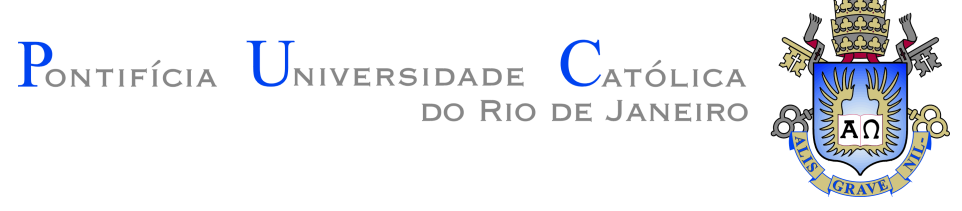

Giovanna Luisa Coelho Leal

\section{A realização de alguns subgrupos discretos do grupo Spin na álgebra de Clifford}

Dissertação apresentada como requisito parcial para obtenção do grau de Mestre pelo Programa de Pós-graduação em Matemática da PUC-Rio. Aprovada pela Comissão Examinadora abaixo:

Prof. Nicolau Corção Saldanha

Orientador

Departamento de Matemática - PUC-Rio

Prof. Emília Carolina Santana Teixeira Alves

Departamento de Matemática Aplicada - UFF

Prof. José Victor Goulart Nascimento

Departamento de Matemática - UFES

Prof. Sergey Galkin

Departamento de Matemática - PUC-Rio

Prof. Thiago Barbosa dos Santos Guerreiro

Departamento de Física - PUC-Rio

Prof. Umberto Leone Hryniewicz

Departamento de Matemática - UFRJ

Rio de Janeiro, 08 de Março de 2021 
Todos os direitos reservados. A reprodução, total ou parcial do trabalho, é proibida sem a autorização da universidade, do autor e do orientador.

\section{Giovanna Luisa Coelho Leal}

Gradou-se em bacharelado e licenciatura em Matemática pela Universidade Federal Fluminense (Rio de Janeiro, Brasil).

Ficha Catalográfica

Leal, G.L.C.

A realização de alguns subgrupos discretos do grupo Spin na álgebra de Clifford / Giovanna Luisa Coelho Leal; orientador: Nicolau Corção Saldanha. - 2021.

72 f: il. color. ; $30 \mathrm{~cm}$

Dissertação (mestrado) - Pontifícia Universidade Católica do Rio de Janeiro, Departamento de Matemática, 2021.

Inclui bibliografia

1. Matemática - Teses. 2. Permutação. 3. Grupo Spin. 4. Álgebra de Clifford. 5. Células de Bruhat. 6. Grupo de Coxeter. I. Saldanha, N.C.. II. Pontifícia Universidade Católica do Rio de Janeiro. Departamento de Matemática. III. Título. 


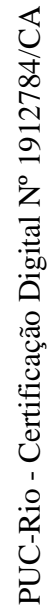

Aos meus pais, Angela e Claudio. 


\section{Agradecimentos}

Agradeço a Deus por estar sempre comigo me dando forças.

A eu mesma por ter conseguido chegar até aqui.

Ao meu orientador Prof. Nicolau Saldanha, por todo o apoio e paciência desde o ínicio do curso de mestrado até a conclusão.

À minha família, que me mantém firme. Em à especial minha irmã Gabrielle, que é minha maior parceira.

Ao meu noivo Natan, por toda compreensão e apoio.

Aos meus amigos, em especial à Camila, Isabela, Guilherme e Raphael que me ajudam e apoiam sempre. Ao Igor Caetano, por todo auxílio e paciência. Ao Nivaldo, por sempre me apoiar e me incentivar.

À CAPES, à Petrobras e a PUC-Rio, pelo suporte financeiro.

O presente trabalho foi realizado com o apoio da Coordenação de Aperfeiçoamento de Pessoal de Nível Superior - Brasil (CAPES) - Código de Financiamento 001. 


\section{Resumo}

Leal, G.L.C.; Saldanha, N.C.. A realização de alguns subgrupos discretos do grupo Spin na álgebra de Clifford. Rio de Janeiro, 2021. 72p. Dissertação de Mestrado - Departamento de Matemática, Pontifícia Universidade Católica do Rio de Janeiro.

A álgebra de Clifford $\mathrm{Cl}_{n+1}^{0}$ é uma álgebra associativa que pode ser realizada matricialmente. O grupo $\operatorname{Spin}_{n+1}$ é uma superfície contida em $\mathrm{Cl}_{n+1}^{0}$ e fechada por multiplicação. Estudamos os geradores de tal grupo, assim como dos grupos de matrizes Quat ${ }_{n+1}$ e $\tilde{\mathrm{B}}_{n+1}^{+}$que são subconjuntos de $\operatorname{Spin}_{n+1}$. Uma permutação em $\mathrm{S}_{n+1}$, pode ser expressa como uma palavra reduzida, por meio de geradores de Coxeter. Os mapas acute e grave nos fornecem elementos em $\tilde{\mathrm{B}}_{n+1}^{+}$, a partir das palavras reduzidas de uma permutação em $\mathrm{S}_{n+1}$. Um elemento da álgebra de Clifford $\mathrm{Cl}_{n+1}^{0}$ pode ser escrito como uma combinação linear de elementos em Quat ${ }_{n+1}$, onde o coeficiente independente é conhecido como parte real. Estudamos resultados que relacionam as características de uma permutação em $\mathrm{S}_{n+1}$, com o elemento a ela relacionado em $\mathrm{Cl}_{n+1}^{0}$.

\section{Palavras-chave}

Permutação; Grupo Spin; Álgebra de Clifford; Células de Bruhat; Grupo de Coxeter. 


\section{Abstract}

Leal, G.L.C.; Saldanha, N.C. (Advisor). The construction of certain discrete subgroups of the Spin group in the Clifford algebra. Rio de Janeiro, 2021. 72p. Dissertação de Mestrado Departamento de Matemática, Pontifícia Universidade Católica do Rio de Janeiro.

The Clifford algebra $\mathrm{Cl}_{n+1}^{0}$ is an associative algebra that can be constructed as an algebra of matrices. The group $\operatorname{Spin}_{n+1}$ is a surface contained in $\mathrm{Cl}_{n+1}^{0}$ and closed by multiplication. We studied the generators of such a group, as well as of the matrix groups Quat $_{n+1}$ and $\tilde{\mathrm{B}}_{n+1}^{+}$which are subsets of $\operatorname{Spin}_{n+1}$. A permutation in $\mathrm{S}_{n+1}$, can be expressed as a reduced word, using transpositions to define the family of Coxeter generators. The acute and grave maps provide us with elements in $\tilde{\mathrm{B}}_{n+1}^{+}$, based on the reduced words of a permutation in $\mathrm{S}_{n+1}$. An element of Clifford algebra $\mathrm{Cl}_{n+1}^{0}$ can be written as a linear combination of elements in Quat ${ }_{n+1}$, where the independent coefficient is known as the real part. We studied results that relate the characteristics of a permutation in $\mathrm{S}_{n+1}$, with the element related to it in $\mathrm{Cl}_{n+1}^{0}$.

\section{Keywords}

Permutation; Spin Group; Clifford Algebra; Bruhat Cell; Coxeter Group. 


\section{Sumário}

1 Introdução 11

1.1 Curvas Localmente Convexas 12

$\begin{array}{lll}1.2 & \text { Estratificação de Bruhat } & 13\end{array}$

$\begin{array}{lll}1.3 & \text { Motivação } & 15\end{array}$

2 Permutações $\quad 17$

2.1 O Grupo Simétrico 17

2.2 Permutações Com Sinais 22

3 Álgebra de Lie $\quad 24$

3.1 A Álgebra de Lie 24

3.2 O Grupo Ortogonal e Sua Álgebra de Lie 26

4 Grupos de Matrizes $\quad 28$

4.1 O Grupo Quat ${ }_{n+1} \quad 28$

4.2 A Álgebra de Clifford $\quad 34$

$\begin{array}{lll}\text { 4.3 O Subgrupo a Um Parâmetro } \alpha_{i}^{\text {Spin }} & 36\end{array}$

4.4 O Grupo $\operatorname{Spin}_{n+1} \quad 40$

4.5 O Grupo $\tilde{\mathrm{B}}_{n+1}^{+} \quad 42$

4.6 O Homomorsfismo П : $\operatorname{Spin}_{n+1} \rightarrow \mathrm{SO}_{n+1}$

5 Permutações e a Álgebra de Clifford $\quad 48$

5.1 Os Mapas Acute e Grave 48

5.2 Parte Real 49

5.2.1 Autovalores e a Parte Real 50

5.2.2 Matrizes de Ciclos e a Parte Real 52

5.2.3 Ciclos e a Parte Real $\quad 55$

$\begin{array}{lll}6 & \text { Sinais } & 61\end{array}$

6.1 Parte Real 61

6.2 Distribuição de Sinais 64

$\begin{array}{lll}7 & \text { Considerações finais } & 68\end{array}$

$\begin{array}{ll}\text { Referências bibliográficas } & 70\end{array}$

$\begin{array}{lll}\text { A Apêndice: Exemplos } & 71\end{array}$

A.1 Permutações Com 1 Ciclo $\quad 71$

A.2 Permutações Com 2 Ciclos 71

A.3 Permutações Com 3 Ciclos $\quad 72$

A.4 Permutações Com 4 Ciclos 72 


\section{Lista de figuras}

Figura 2.1 Diagrama de $\eta \in \mathrm{S}_{4} \quad 21$

Figura 2.2 Diagramas de $\sigma=a_{2} a_{3} a_{2} a_{1}=a_{3} a_{2} a_{3} a_{1} \in \mathrm{S}_{4} \quad 21$

Figura 2.3 Diagrama de $\sigma=a_{1} a_{2} a_{1} \in \mathrm{S}_{3} \quad 22$

Figura 4.1 Diagrama de $\sigma=a_{3} \in \mathrm{S}_{4} \quad 46$

Figura 5.1 Diagrama de $\eta \in \mathrm{S}_{6} \quad 49$

Figura 5.2 Diagrama de $\sigma=a_{2} a_{1} a_{3} \in \mathrm{S}_{4} \quad 50$

Figura 5.3 Diagrama de $\sigma=a_{1} a_{2} a_{3} a_{2} a_{1} a_{4} a_{3} a_{2} a_{1} \in \mathrm{S}_{5} \quad 56$

Figura 5.4 Diagrama de $\sigma=a_{2} a_{1} a_{3} a_{2} \in \mathrm{S}_{4} \quad 58$

Figura 6.1 Diagrama de $\sigma=a_{2} a_{1} a_{3} a_{2} a_{3} \in \mathrm{S}_{4} \quad 62$

Figura 6.2 Diagrama de $\sigma=a_{1} a_{3} a_{2} \in \mathrm{S}_{4} \quad 62$

Figura 6.3 Diagrama de $\sigma=a_{1} a_{4} a_{2} a_{5} \in \mathrm{S}_{6} \quad 63$

Figura 6.4 Diagrama de $\sigma=a_{5} a_{3} a_{4} a_{2} a_{1} \in \mathrm{S}_{6} \quad 63$

Figura 6.5 Diagrama de $\sigma=a_{1} a_{2} a_{3} \in \mathrm{S}_{4} \quad 64$ 
O medo precede a coragem.

James Lake, Caçadores de Trolls: Contos de Arcadia. 


\section{1 \\ Introdução}

O presente trabalho tem como objetivo, descrever os grupos Quat ${ }_{n+1}$, $\tilde{\mathrm{B}}_{n+1}^{+}$e $\operatorname{Spin}_{n+1}$ como grupos de matrizes, para então estudar permutações $\sigma \in \mathrm{S}_{n+1}$ nas coordenadas de $z \in \tilde{\mathrm{B}}_{n+1}^{+} \subset \operatorname{Spin}_{n+1}$. A inspiração para o trabalho vem de [1].

O objetivo provém do estudo das curvas localmente convexas na esfera $\mathbb{S}^{n}$, levando assim ao interesse de tais curvas no grupo $\operatorname{Spin}_{n+1}$.

Tal grupo, pode ser decomposto em células de Bruhat da seguinte forma:

$$
\operatorname{Spin}_{n+1}=\bigsqcup_{z \in \tilde{\mathrm{B}}_{n+1}^{+}} \operatorname{Bru}_{z}
$$

esta decomposição é chamada estratificação de Bruhat do grupo $\operatorname{Spin}_{n+1}$.

A união das células de Bruhat com sinal $\mathrm{Bru}_{z}$ com $z \in \tilde{\mathrm{B}}_{n+1}^{+}$tal que $\Pi(z)=P_{\sigma} \in \mathrm{SO}_{n+1}$ é a célula de Bruhat sem sinal $\mathrm{Bru}_{\sigma} \subset \mathrm{Spin}_{n+1}$, onde $\sigma \in \mathrm{S}_{n+1}$. Cada componente conexa de uma célula de Bruhat sem sinal contém exatamente um elemento $z \in \tilde{\mathrm{B}}_{n+1}^{+} \subset \operatorname{Spin}_{n+1}$.

Dessa forma, podemos compreender melhor o grupo $\operatorname{Spin}_{n+1}$ através das células de Bruhat. Assim, a estratificação se mostrou uma ferramenta essencial no estudo das curvas localmente convexas no grupo $\operatorname{Spin}_{n+1}[5]$.

Primeiramente, ainda na introdução, daremos uma breve explicação dos conceitos mencionados anteriormente, que guiaram o objetivo do trabalho.

No capítulo 2, faremos um breve resumo sobre o grupo de permutações $\mathrm{S}_{n+1}$, assim como o grupo das matrizes de permutações com sinal $\mathrm{B}_{n+1}$.

No capítulo 3, vamos ver um pouco sobre álgebras de Lie, mais precisamente, álgebras de Lie de matrizes, com o foco voltado para a álgebra de Lie $\mathfrak{s o}_{n+1}$ do grupo de matrizes ortogonais $\mathrm{SO}_{n+1}$.

No capítulo 4, faremos primeiramente a construção matricial do grupo Quat $_{n+1}$ e de seus geradores $\hat{a}_{i}$, com $i \in \llbracket n \rrbracket=\{1, \ldots, n\}$. Em seguida, construiremos a álgebra de Clifford $\mathrm{Cl}_{n+1}^{0}$, que é uma álgebra associativa com unidade, cuja base ortonormal é formada pelos elementos de um subconjunto HQuat $_{n+1} \subset$ Quat $_{n+1}$.

Após a construção de $\mathrm{Cl}_{n+1}^{0}$, faremos a construção matricial do grupo $\operatorname{Spin}_{n+1}$, que é gerado pelos elementos $\alpha_{i}(\theta)$, onde $\alpha_{i}: \mathbb{R} \rightarrow \operatorname{Spin}_{n+1}$ é um 
subgrupo a um parâmetro, com $\alpha_{i}(\pi)=\hat{a}_{i}$. Além disso, vale ressaltar que Quat $_{n+1} \subset \operatorname{Spin}_{n+1} \subset \mathrm{Cl}_{n+1}^{0}$.

Tendo construídos tais grupos, faremos a construção do nosso último grupo de interesse $\tilde{\mathrm{B}}_{n+1}^{+} \subset \operatorname{Spin}_{n+1} \subset \mathrm{Cl}_{n+1}^{0}$, que é o grupo de matrizes gerado pelos elementos $\alpha_{i}\left(\frac{\pi}{2}\right)=\dot{a}_{i}$.

Ainda no capítulo 4, analisaremos a ação do homomorfismo

$$
\Pi: \operatorname{Spin}_{n+1} \rightarrow \mathrm{SO}_{n+1}
$$

nos geradores dos grupos que construímos nas seções anteriores.

No capítulo 5, estudaremos as permutações $\sigma \in \mathrm{S}_{n+1}$ por meio das coordenadas de $z \in \tilde{\mathrm{B}}_{n+1}^{+} \subset \operatorname{Spin}_{n+1}$. Estudaremos primeiramente, o funcionamento dos mapas acute, grave : $\mathrm{S}_{n+1} \rightarrow \tilde{\mathrm{B}}_{n+1}^{+}$, para enfim estudarmos resultados que relacionam a parte real de elementos da álgebra de Clifford $\mathrm{Cl}_{n+1}^{0}$, com as características da permutação a ele relacionada.

Por fim, no capítulo 6, faremos algumas afirmações sobre o comportamento de determinados elementos em $\tilde{\mathrm{B}}_{n+1}^{+} \subset \operatorname{Spin}_{n+1}$.

Notação 1.0.1 As notações para os elementos $\dot{a}_{i}, \grave{a}_{i}, \hat{\sigma}, \grave{\sigma} \in \tilde{\mathrm{B}}_{n+1}^{+}, \hat{a}_{i} \in \mathrm{Cl}_{n+1}^{0}$, bem como $\alpha_{i} \in \operatorname{Spin}_{n+1}$, foram pensadas pelo orientador e seus colaboradores para os trabalhos anteriores. Pode-se identificar $\hat{a}_{i} \in \mathrm{Cl}_{n+1}^{0}$ como $\hat{a}_{i}=e_{j+1} e_{j}$, na notação de [2].

\section{1}

\section{Curvas Localmente Convexas}

Para $j \in \llbracket n \rrbracket$, considere as matrizes $\mathfrak{a}_{j}^{\mathrm{SO}}=e_{j+1} e_{j}^{T}-e_{j} e_{j+1}^{T} \in \mathfrak{s o}_{n+1}$, note que $\mathfrak{a}_{j}^{\text {SO }}$ é anti-simétrica. Como $\mathfrak{s o}_{n+1}$ e $\mathfrak{s p i n}_{n+1}$ são isomorfas, podemos identificar tais elementos na álgebra de Lie $\mathfrak{s p i n}_{n+1}$ como

$$
\mathfrak{a}_{j}^{\text {Spin }}=\frac{1}{2} \hat{a}_{j}
$$

onde $\hat{a}_{i} \in$ Quat $_{n+1} \subset \operatorname{Spin}_{n+1}$, tal elemento será apresentado mais tarde.

Notação 1.1.1 Representaremos $\mathfrak{a}_{j}^{\text {Spin }} \in \mathfrak{s p i n}_{n+1}$ e $\mathfrak{a}_{j}^{\mathrm{SO}} \in \mathfrak{s o}_{n+1}$ por $\mathfrak{a}_{j}$ quando estiver implícita a álgebra de Lie de origem.

Uma curva $\Gamma: J \rightarrow \operatorname{Spin}_{n+1} \operatorname{com} J \subset \mathbb{R}$, é dita localmente convexa se é absolutamente contínua, e sua derivada logarítmica tem a forma

$$
(\Gamma(t))^{-1} \Gamma^{\prime}(t)=\sum_{j \in \llbracket n \rrbracket} k_{j}(t) \mathfrak{a}_{j}
$$


onde $k_{1}, \ldots, k_{n}: J \rightarrow(0,+\infty)$ são funções positivas.

Dada uma curva localmente convexa suave $\Gamma$, a curva suave

$$
\gamma: J \rightarrow \mathbb{R}^{n+1}, \quad \gamma(t)=\Pi(\Gamma(t)) e_{1},
$$

satisfaz a desigualdade $\operatorname{det}\left(\gamma(t), \gamma^{\prime}(t), \ldots, \gamma^{n}(t)\right)>0$, onde

$$
\Pi: \operatorname{Spin}_{n+1} \rightarrow \mathrm{SO}_{n+1}
$$

é um mapa de recobrimento.

Uma curva paramétrica $\gamma: J \rightarrow \mathbb{R}^{n+1}$ satisfazendo a desigualdade acima, é também chamada de localmente convexa. Tal curva $\gamma$, pode ser levantada para uma curva localmente convexa $\mathfrak{F}_{\gamma}$ em $\mathrm{SO}_{n+1}$, consequentemente em $\operatorname{Spin}_{n+1}$, tomando a matriz ortogonal $\mathfrak{F}_{\gamma}(t)$, cujos vetores coluna são os resultados de aplicar o algoritmo de Gram-Schmidt na base ordenada $\left(\gamma(t), \gamma^{\prime}(t), \ldots, \gamma^{n}(t)\right)$ de $\mathbb{R}^{n+1}$.

A base ortogonal obtida é o frame de Frenet generalizado do espaço de curvas $\gamma$. Além disso, os coeficientes $k_{1}, \ldots, k_{n}$ da derivada logarítmica de $\mathfrak{F}_{\gamma}$ são as curvaturas generalizadas de $\gamma$ (multiplicadas pela velocidade $v_{\gamma}=\left|\gamma^{\prime}\right|$ ).

\section{2}

\section{Estratificação de Bruhat}

A decomposição de Bruhat é uma forma de escrever uma matriz como um produto de matrizes especiais. Podendo assim, decompor um grupo de matrizes em uma união disjunta de células de Bruhat.

Chamamos de $\mathrm{B}_{n+1}$ o grupo das matrizes de permutações com sinal, $\mathrm{Up}_{n+1}$ o grupo das matrizes triangulares superiores e $\mathrm{Up}_{n+1}^{+}$quando a diagonal é positiva.

Teorema 1.2.1 Dado $M \in \mathrm{GL}_{n+1}$, existe uma única permutação $\sigma \in \mathrm{S}_{n+1}$ e $U_{0}, U_{1} \in \mathrm{Up}_{n+1}$ (não únicas), tais que

$$
M=U_{0} P_{\sigma} U_{1}
$$

onde $P_{\sigma} \in \mathrm{S}_{n+1} \subset \mathrm{B}_{n+1}$. Em particular,

$$
\mathrm{GL}_{n+1}=\bigsqcup_{\sigma \in \mathrm{S}_{n+1}} \mathrm{Up}_{n+1} P_{\sigma} \mathrm{Up}_{n+1}
$$

Observe que os subconjuntos $\mathrm{Up}_{n+1} P_{\sigma} \mathrm{Up}_{n+1}$ são classes laterais duplas.

Podemos absorver os sinais de $U_{0}$ e $U_{1}$ em $P_{\sigma}$, gerando assim a decomposição de Bruhat com sinal: para toda $M \in \mathrm{GL}_{n+1}$, existe uma única matriz 
de permutação com sinais $P \in \mathrm{B}_{n+1}$ e $\tilde{U}_{0}, \tilde{U}_{1} \in \mathrm{Up}_{n+1}^{+}$(não únicas), tal que $M=\tilde{U}_{0} P \tilde{U}_{1}$.

Observação 1.2.1 Pode-se formular o teorema para matrizes triangulares inferiores da mesma forma.

Para cada $P \in \mathrm{B}_{n+1}$, a classe lateral dupla de $\mathrm{Up}_{n+1}^{+}$é um subconjunto contrátil de $\mathrm{GL}_{n+1}$. Chamamos de célula de Bruhat com sinal a interseção da classe com o grupo ortogonal. Cada célula de Bruhat com sinal é ainda uma subvariedade contrátil do grupo ortogonal.

A decomposição de $\mathrm{SO}_{n+1}$ em células de Bruhat é conhecida como estratificação de Bruhat com sinais e é dada por

$$
\mathrm{SO}_{n+1}=\bigsqcup_{P \in \mathrm{B}_{n+1}^{+}} \operatorname{Bru}_{P}, \quad \mathrm{Bru}_{P}=\left(\mathrm{Up}_{n+1}^{+} P \mathrm{Up}_{n+1}^{+}\right) \cap \mathrm{SO}_{n+1}, \quad P \in \mathrm{B}_{n+1}^{+},
$$

onde $\mathrm{B}_{n+1}^{+}=\mathrm{B}_{n+1} \cap \mathrm{SO}_{n+1}$.

A pré imagem de cada célula pelo mapa de recobrimento $\Pi: \operatorname{Spin}_{n+1} \rightarrow$ $\mathrm{SO}_{n+1}$ é uma união disjunta de duas componentes contráteis.

Para $z \in \tilde{\mathrm{B}}_{n+1}^{+}=\Pi^{-1}\left[\mathrm{~B}_{n+1}^{+}\right]$, seja $\mathrm{Bru}_{z}$ a componente conexa de $\Pi^{-1}\left[\operatorname{Bru}_{\Pi(z)}\right]$ contendo $z$. Chamamos $\mathrm{Bru}_{z}$ de célula de Bruhat com sinal. A célula de Bruhat sem sinal $\mathrm{Bru}_{\sigma} \subset \operatorname{Spin}_{n+1}$ é a união disjunta das células $\mathrm{Bru}_{z}$, com $z \in \tilde{\mathrm{B}}_{n+1}^{+}$.

Para $z \in \operatorname{Spin}_{n+1}$ e $\sigma \in \mathrm{S}_{n+1}$, temos $z \in \mathrm{Bru}_{\sigma}$ se e somente se, existem $U_{1}, U_{2} \in \mathrm{Up}_{n+1}$ e $P_{\sigma} \in \mathrm{B}_{n+1}$, tal que $\Pi(z)=U_{1} P_{\sigma} U_{2}$.

Portanto, a estratificação de Bruhat do grupo $\operatorname{Spin}_{n+1}$ é

$$
\operatorname{Spin}_{n+1}=\bigsqcup_{z \in \tilde{\mathrm{B}}_{n+1}^{+}} \operatorname{Bru}_{z}
$$

Para todo $U \in \mathrm{Up}_{n+1}^{+}$e $Q \in \mathrm{SO}_{n+1}$, seja $Q^{U}=\mathbf{Q}\left(U^{-1} Q\right)$ uma $\mathrm{Up}_{n+1^{-}}^{+}$ ação. As células de Bruhat em $\mathrm{SO}_{n+1}$ podem ser vistas como órbitas desta $\mathrm{Up}_{n+1}^{+}$-ação.

Tal ação preserva células de Bruhat e pode ser levantada para uma ação em $\operatorname{Spin}_{n+1}$, tomando $z^{U}=\mathbf{Q}\left(U^{-1} z\right)$. As células de Bruhat, assim como anteriormente, podem ser vistas como órbitas desta $\mathrm{Up}_{n+1}^{+}$-ação.

Além disso, se $U \in \mathrm{Up}_{n+1}^{+}$e $\Gamma:[0,1] \rightarrow \operatorname{Spin}_{n+1}$ é localmente convexa, então $\Gamma^{U}:[0,1] \rightarrow \operatorname{Spin}_{n+1}, \operatorname{com}^{U}=\mathbf{Q}\left(U^{-1} \Gamma(t)\right)$ é também uma curva localmente convexa. 


\section{3 \\ Motivação}

Nesta seção, vamos falar um pouco sobre os resultados que motivaram o presente trabalho. As construções detalhadas, assim como as demonstrações dos resultados, foram feitas pelo orientador juntamente com seus colaboradores e podem ser encontradas em [5].

O grupo Quat gut $_{n+1}$ age livre e transitivamente por multiplicação a esquerda, sobre a coleção das componentes conexas de uma célula de Bruhat sem sinal.

Portanto, o resultado a seguir, gera uma parametrização explícita para todas as células de Bruhat com sinais do grupo $\operatorname{Spin}_{n+1}$.

Teorema 1.3.1 Dadas palavras reduzidas $a_{i_{1}} \cdots \cdots a_{i_{k}}<a_{i_{1}} \cdots \cdots a_{i_{k}} a_{j}$ para duas permutações consecutivas em $\mathrm{S}_{n+1}$ e dados sinais $\varepsilon_{1}, \ldots \varepsilon_{k}, \varepsilon \in\{ \pm 1\}$, defina

$$
\begin{gathered}
z_{1}=\left(\dot{a}_{i_{1}}\right)^{\varepsilon_{1}} \cdots \cdot\left(\dot{a}_{i_{k}}\right)^{\varepsilon_{k}} \\
z_{0}=z_{1}\left(\dot{a}_{j}\right)^{\varepsilon} \in \widetilde{\mathrm{B}}_{n+1}^{+} .
\end{gathered}
$$

Dado $q \in$ Quat $_{n+1}$, o mapa $\Psi: \operatorname{Bru}_{q z_{1}} \times(0, \pi) \rightarrow \operatorname{Bru}_{q z_{0}}$ dado por $\Psi(z, \theta)=$ $z \alpha_{j}(\varepsilon \theta)$ é um difeomorfismo.

Ao conseguirmos descrever a estratificação de Bruhat do grupo $\operatorname{Spin}_{n+1}$, tornamos o estudo das curvas localmente convexas um pouco menos complexo, pois agora temos a álgebra das células de Bruhat a nosso favor.

Um exemplo de um resultado que relaciona as células de Bruhat com as curvas localmente convexas é o teorema a seguir, o resultado discute a transversalidade entre as curvas localmente convexas e as células de Bruhat.

Teorema 1.3.2 Considere $z_{0} \in \tilde{\mathrm{B}}_{n+1}^{+} \subset \operatorname{Spin}_{n+1}, \sigma=\sigma_{z_{0}} \in \mathrm{S}_{n+1}, \sigma \neq \eta$, $k=\operatorname{inv}(\eta)-\operatorname{inv}(\sigma)>0$, onde $\eta \in \mathrm{S}_{n+1}$ é o elemento com número máximo de inversões. Então, existe uma vizinhança aberta $\mathcal{U}_{z_{0}}$ da célula de Bruhat com sinal não aberta $\mathrm{Bru}_{z_{0}}$ em $\operatorname{Spin}_{n+1}$ e um mapa suave

$$
f=\left(f_{1}, \ldots, f_{k}\right): \mathcal{U}_{z_{0}} \rightarrow \mathbb{R}^{k}
$$

com as seguintes propriedades:

(i) Para todo $z \in \mathcal{U}_{z_{0}}$, tem-se que $z \in \mathrm{Bru}_{z_{0}}$ se, e somente se $f(z)=0$;

(ii) Para todo $z \in \mathcal{U}_{z_{0}}$, a derivada $D f(z)$ é sobrejetora; 
(iii) Para qualquer curva localmente convexa suave $\Gamma:(-\epsilon, \epsilon) \rightarrow \mathcal{U}_{z_{0}}$, tem-se

$$
\left(f_{k} \circ \Gamma\right)^{\prime}(t)>0
$$

para todo $t \in(-\epsilon, \epsilon)$.

Outros resultados importantes, podem ser encontrados em [5].

Tendo em vista os resultados anteriores, podemos perceber a importância das células de Bruhat no estudo das curvas localmente convexas. Assim, fica clara a necessidade de compreendermos os elementos de tais células, que nada mais são do que elementos do grupo $\operatorname{Spin}_{n+1}$. 


\section{2}

\section{Permutações}

O grupo $\mathrm{S}_{n+1}$ das permutações de $n+1$ elementos é gerado pelas transposições $a_{i}=(i, i+1)$, com $i \in \llbracket n \rrbracket$, onde $\llbracket n \rrbracket=\{1, \ldots, n\}$. Tais geradores nos proporcionam escrever uma palavra reduzida para $\sigma \in \mathrm{S}_{n+1}$.

Além disso, uma permutação $\sigma \in \mathrm{S}_{n+1}$ pode ser associada a uma matriz $P_{\sigma}$, chamada de matriz de permutação.

Neste capítulo, estudaremos o grupo de permutações $\mathrm{S}_{n+1}$, as matrizes de permutação e por fim, o grupo de matrizes de permutação com sinais $\mathrm{B}_{n+1}$.

\section{1}

\section{O Grupo Simétrico}

Uma permutação de um conjunto é uma função bijetiva, que leva um elemento do conjunto em outro elemento também no conjunto, não necessariamente diferente do original.

O grupo de permutações de um conjunto $X$ é o conjunto de bijeções $f: X \rightarrow X$ que com a operação de composição de funções, formam um grupo.

Seja $\mathrm{S}_{n+1}$ o grupo das permutações do conjunto $\llbracket n+1 \rrbracket=\{1, \ldots, n+1\}$. Denotamos a ação de $\mathrm{S}_{n+1}$ em $\llbracket n+1 \rrbracket$ por

$$
(\sigma, k) \mapsto k^{\sigma}
$$

Escrevemos a composta da esquerda para a direita, de tal forma que temos

$$
k^{\sigma_{1} \sigma_{2}}=\left(k^{\sigma_{1}}\right)^{\sigma_{2}}
$$

Definição 2.1.1 $\mathrm{S}_{n+1}$ é o grupo gerado pelas $n$ transposições $a_{i}=(i, i+1)$, com $i \in \llbracket n \rrbracket$.

Observação 2.1.1 Os geradores $a_{i}=(i, i+1)$ são conhecidos como geradores de Coxeter-Weyl. Além disso, o grupo $\mathrm{S}_{n+1}$ pode ser pensado também como o grupo $A_{n}$ de Coxeter-Weyl, entretanto não abordaremos tal interpretação (veja, $[6])$.

Exemplo 2.1.1 Sejam $\sigma_{1}=[3421]$ e $\sigma_{2}=[4132] \in \mathrm{S}_{4}$. Como $\sigma_{1} \sigma_{2}=$ $\left[1^{\sigma_{1} \sigma_{2}} 2^{\sigma_{1} \sigma_{2}} 3^{\sigma_{1} \sigma_{2}} 4^{\sigma_{1} \sigma_{2}}\right]$, ent $\tilde{a} o \sigma_{1} \sigma_{2}=[3214]$. 
Escrevemos uma permutação $\sigma \in \mathrm{S}_{n+1}$ usualmente de duas formas, como uma lista de valores $\left[1^{\sigma} 2^{\sigma} \ldots n^{\sigma}\right]$, assim como feito no exemplo acima, ou como um produto de transposições, por exemplo $\sigma=a_{i_{1}} \ldots a_{i_{k}}$.

Definição 2.1.2 Um ciclo é uma permutação $\sigma=\left(i_{1} \ldots i_{k}\right) \in \mathrm{S}_{n+1}$, onde $i_{k} \mapsto i_{1}, i_{j} \mapsto i_{j+1}$ se $1 \leq j<k$ e $i_{l} \mapsto i_{l}$ caso contrário.

Proposição 2.1.1 Toda permutação $\sigma \in \mathrm{S}_{n+1}$ pode ser escrita como um produto de ciclos disjuntos, de forma única.

A demonstração da proposição será omitida, a mesma pode ser encontrada em [3].

Exemplo 2.1.2 Seja $\sigma=[24513] \in \mathrm{S}_{5}$, visto que $1 \mapsto 2 \mapsto 4$ e $3 \mapsto 5$, $\sigma$ é escrita como $\sigma=(124)(35)$.

Definição 2.1.3 O conjunto $\operatorname{Inv}(\sigma)=\left\{(i, j) \in \llbracket n+1 \rrbracket^{2} /(i<j) \wedge\left(i^{\sigma}>j^{\sigma}\right)\right\}$ é o conjunto dos pares $(i, j)$ que são inversões de $\sigma$. Além disso, $\operatorname{inv}(\sigma)=$ $\operatorname{card}\{\operatorname{Inv}(\sigma)\}$.

Exemplo 2.1.3 Seja $\sigma \in \mathrm{S}_{5}$ tal que $\sigma=[24153]=a_{2} a_{4} a_{1} a_{3}$, então $\operatorname{Inv}(\sigma)=$ $\{(1,3),(2,3),(2,5),(4,5)\}$, assim $\operatorname{inv}(\sigma)=4$.

Definição 2.1.4 Uma palavra reduzida para uma permutação $\sigma \in \mathrm{S}_{n+1}$ é uma expressão de $\sigma$ como um produto de geradores $a_{i}=(i, i+1)$, tal que a quantidade seja minimal e igual a $\operatorname{inv}(\sigma)$.

No exemplo acima, temos uma permutação já escrita como uma palavra reduzida, visto que $\sigma=a_{2} a_{4} a_{1} a_{3}$ possui quatro geradores e quatro inversões.

Definição 2.1.5 Seja $\sigma \in \mathrm{S}_{n+1}$, definimos sua matriz de permutação $P_{\sigma}$ por $e_{k}^{T} P_{\sigma}=e_{k^{\sigma}}^{T}$.

Exemplo 2.1.4 Seja $\sigma=a_{2} a_{4} a_{1} a_{3}$ assim como no exemplo 2.1.3, então

$$
P_{\sigma}=\left(\begin{array}{lllll}
0 & 1 & 0 & 0 & 0 \\
0 & 0 & 0 & 1 & 0 \\
1 & 0 & 0 & 0 & 0 \\
0 & 0 & 0 & 0 & 1 \\
0 & 0 & 1 & 0 & 0
\end{array}\right)
$$


Existe uma única permutação $\eta=a_{1} a_{2} a_{1} a_{3} a_{2} a_{1} \ldots a_{n} a_{n-1} \ldots a_{2} a_{1}$, tal que o comprimento da palavra reduzida $\operatorname{inv}(\eta)=\frac{n(n+1)}{2}$ é o máximo possível, sua matriz é dada por

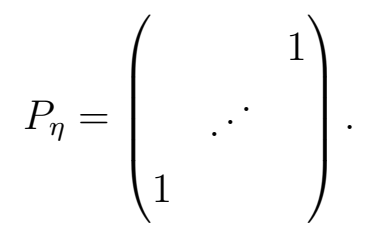

Proposição 2.1.2 Para todo $i, j \in \llbracket n \rrbracket$, temos:

(i) $a_{j} a_{i}=a_{i} a_{j}$, para $|i-j| \neq 1$;

(ii) $a_{i} a_{i+1} a_{i}=a_{i+1} a_{i} a_{i+1}$.

Prova.

Para a primeira igualdade, é simples ver que dados $a_{i}$ e $a_{j} \operatorname{com}|i-j| \neq 1$, temos que $i \neq j+1$ e $j \neq i+1$. Portanto fazer primeiro $a_{i}$ ou $a_{j}$ não altera o resultado, visto que os geradores são disjuntos.

Para a segunda igualdade, basta pensar na permutação representada como lista de números,

$$
\begin{aligned}
a_{i} a_{i+1} a_{i} & =\left[\left(\left(i^{a_{i}}\right)^{a_{i+1}}\right)^{a_{i}}\left(\left((i+1)^{a_{i}}\right)^{a_{i+1}}\right)^{a_{i}}\left(\left((i+2)^{a_{i}}\right)^{a_{i+1}}\right)^{a_{i}}\right] \\
& =\left[\left((i+1)^{a_{i+1}}\right)^{a_{i}}\left((i)^{a_{i+1}}\right)^{a_{i}}\left((i+2)^{a_{i+1}}\right)^{a_{i}}\right] \\
& =\left[(i+2)^{a_{i}}(i)^{a_{i}}(i+1)^{a_{i}}\right] \\
& =\left[\begin{array}{lll}
(i+2) & (i+1) & i
\end{array}\right],
\end{aligned}
$$

da mesma forma podemos ver que

$$
\begin{aligned}
& a_{i+1} a_{i} a_{i+1}=\left[\left(\left(i^{a_{i+1}}\right)^{a_{i}}\right)^{a_{i+1}}\left(\left((i+1)^{a_{i+1}}\right)^{a_{i}}\right)^{a_{i+1}}\left(\left((i+2)^{a_{i+1}}\right)^{a_{i}}\right)^{a_{i+1}}\right] \\
& =\left[\begin{array}{lll}
(i+2) & (i+1) & i
\end{array}\right] .
\end{aligned}
$$

Portanto $a_{i} a_{i+1} a_{i}=a_{i+1} a_{i} a_{i+1}$.

Teorema 2.1.1 Duas palavras reduzidas para uma permutação estão conectadas por uma sequência finita de movimentos dos tipos acima.

Prova.

Dividiremos a demonstração em casos e procederemos por indução no comprimento $l=\operatorname{inv}(\sigma)$.

Os casos $l=0,1,2$ são triviais. Suponhamos por indução que o enunciado seja válido para valores menores que 1 . 
Sejam $w_{1}=a_{i_{1}} \ldots a_{i_{n}}$ e $w_{2}=a_{j_{1}} \ldots a_{j_{n}}$ duas palavras reduzidas para $\sigma \in \mathrm{S}_{n+1}, \operatorname{com} n=\operatorname{inv} \sigma$.

Se a primeira letra de $w_{1}$ é igual a primeira letra de $w_{2}$, ou seja $a_{i_{1}}=a_{j_{1}}$, temos que $w_{1}=a_{i_{1}} \tilde{w}_{1}$ e $w_{2}=a_{i_{1}} \tilde{w}_{2}$, onde $\tilde{w}_{1}$ e $\tilde{w}_{2}$ são palavras reduzidas para $\tilde{\sigma}, \operatorname{com} \sigma=a_{i_{1}} \tilde{\sigma}$.

Pela hipótese de indução $\tilde{w}_{1}$ e $\tilde{w}_{2}$ estão conectadas, portanto $w_{1}$ e $w_{2}$ também estão.

Se a primeira letra de $w_{1}$ e a primeira letra de $w_{2}$ são tais que $\left|i_{1}-j_{1}\right|>1$, então $w_{1}=a_{i_{1}} \tilde{w}_{1}$ e $w_{2}=a_{j_{1}} \tilde{w}_{2}$, onde $\tilde{w}_{1}$ e $\tilde{w}_{2}$ são palavras reduzidas para $\sigma_{1}$ e $\sigma_{2}$ respectivamente, com $\sigma=a_{i_{1}} \sigma_{1}$ e $\sigma=a_{j_{1}} \sigma_{2}$.

Seja $\tilde{\sigma}$ tal que $\sigma_{1}=a_{j_{1}} \tilde{\sigma}$ e $\sigma_{2}=a_{i_{1}} \tilde{\sigma}$. Sejam $w_{3}$ e $w_{4}$ outras palavras reduzidas para $\sigma_{1}$ e $\sigma_{2}$ respectivamente, tais que $w_{3}=a_{j_{1}} \tilde{w}_{3}$, onde $\tilde{w}_{3}$ é outra palavra reduzida para $\tilde{\sigma}$ e $w_{4}=a_{j_{1}} \tilde{w}_{4}$, onde $\tilde{w}_{4}$ é outra palavra reduzida para $\tilde{\sigma}$.

Por indução $\tilde{w}_{1}$ e $w_{3}$ estão conectadas, assim como $\tilde{w}_{2}$ e $w_{4}$. Portanto, também por indução, $\tilde{w}_{3}$ e $\tilde{w}_{4}$ estão conectadas. Assim, conectamos $w_{1}$ e $w_{2}$.

Por último, suponhamos que a primeira letra de $w_{2}$ é tal que $a_{j_{1}}=a_{i_{1}+1}$, onde $a_{i_{1}}$ é a primeira letra de $w_{1}$. Assim, pelos argumentos anteriores, existe $\tilde{\sigma}$ tal que $\sigma=a_{i_{1}} a_{i_{1}+1} a_{i_{1}} \tilde{\sigma}=a_{i_{1}+1} a_{i_{1}} a_{i_{1}+1} \tilde{\sigma}$.

Se $w_{1}=a_{i_{1}} \tilde{w}_{1}$, conectamos $\tilde{w}_{1}$ a $a_{i_{1}+1} a_{i_{1}} w_{3}$. Se $w_{2}=a_{i_{1}+1} \tilde{w}_{2}$, conectamos $\tilde{w}_{2}$ a $a_{i_{1}} a_{i_{1}+1} w_{4}$. Note que $w_{3}$ e $w_{4}$ são palavras reduzidas de $\tilde{\sigma}$, portanto podemos conectá-las, por indução. Assim, conectamos $w_{1}$ e $w_{2}$.

Logo, podemos conectar quaisquer duas palavras reduzidas de uma permutação $\sigma \in \mathrm{S}_{n+1}$, por meio dos movimentos que conhecemos.

Exemplo 2.1.5 Seja $\sigma=a_{2} a_{1} a_{3} a_{4} a_{3} a_{5} \in \mathrm{S}_{6}$, fazendo uso da proposição anterior, podemos escrever mais de uma palavra reduzida que descreve a permutação. Como por exemplo:

$\sigma=a_{2} a_{1} a_{4} a_{3} a_{4} a_{5}, \sigma=a_{2} a_{4} a_{1} a_{3} a_{4} a_{5}$ e $\sigma=a_{2} a_{4} a_{3} a_{1} a_{4} a_{5}$.

Por uma verificação simples vemos que as 4 palavras reduzidas representam a permutação $\sigma=[261435] \in \mathrm{S}_{6}$.

As palavras reduzidas para uma permutação $\sigma$ podem ser representadas por meio de um diagrama. Existe mais de uma forma de ler tal diagrama. No nosso caso, cada ponto representa um número começando em 1 e terminando em $n$, de cima para baixo, onde a permutação é lida levando os pontos da esquerda nos pontos da direita. Cada interseção representa um gerador $a_{i}$, da esquerda para a direita. Além disso, de cima para baixo, o espaço entre dois pontos faz referência a um único gerador, começando em $a_{1}$ e indo até $a_{n}$. 
Utilizamos esta representação para encontrar palavras reduzidas para as permutações.

Vamos ver alguns exemplos.

Exemplo 2.1.6 Seja $\eta=$ [4321], construímos o diagrama de $\eta$ marcando os pontos como descrito acima. Dessa forma, levamos o primeiro ponto à esquerda ao último ponto à direita, seguindo a permutação da mesma forma com os outros pontos. Assim, temos

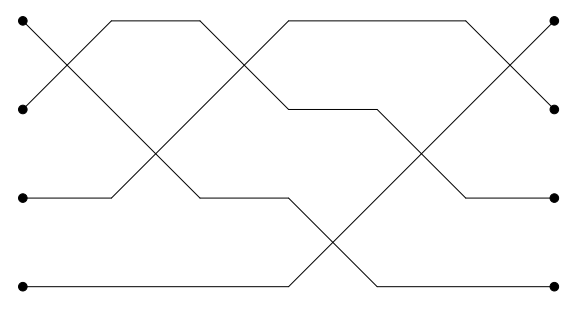

Figura 2.1: Diagrama de $\eta \in \mathrm{S}_{4}$

Agora só precisamos ler o diagrama. Como descrito acima, os geradores são lidos de cima para baixo e da esquerda para a direita. Portanto, temos que a palavra reduzida de $\eta$ é dada por

$$
\eta=a_{1} a_{2} a_{1} a_{3} a_{2} a_{1}
$$

O próximo exemplo ilustra dois diagramas diferentes para uma única permutação.

Exemplo 2.1.7 Seja $\sigma=[2431]=a_{2} a_{3} a_{2} a_{1}=a_{3} a_{2} a_{3} a_{1}$, seus diagramas são respectivamente

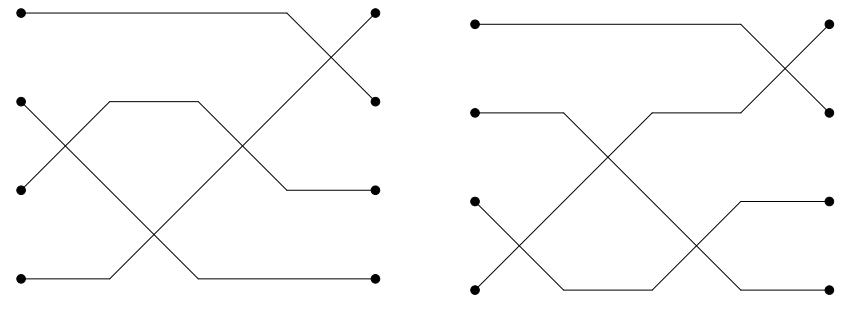

Figura 2.2: Diagramas de $\sigma=a_{2} a_{3} a_{2} a_{1}=a_{3} a_{2} a_{3} a_{1} \in \mathrm{S}_{4}$

Note que o que mudou no diagrama foi a inversão de 3 geradores, que equivalem a $a_{2} a_{3} a_{2}$ e $a_{3} a_{2} a_{3}$, respectivamente. Pela proposição anterior, sabemos que são iguais. 


\section{2}

\section{Permutações Com Sinais}

Vimos na seção anterior que uma permutação $\sigma \in \mathrm{S}_{n+1}$ está associada a uma matriz $(n+1) \times(n+1)$, denominada $P_{\sigma}$. Por outro lado, um certo tipo de matriz também pode ser associado a uma permutação. Nesta seção, vamos estudar tais grupos de matrizes.

Seja $\mathrm{B}_{n+1}$ o grupo das matrizes de permutações com sinal, ou seja, matrizes ortogonais $\mathrm{P}$ tais que existe uma permutação $\sigma \in \mathrm{S}_{n+1}$, onde

$$
e_{i}^{T} P= \pm e_{i^{\sigma}}^{T}, \quad \forall i \in \llbracket n+1 \rrbracket .
$$

Exemplo 2.2.1 Seja a matriz

$$
P=\left(\begin{array}{ccc}
0 & 0 & 1 \\
0 & -1 & 0 \\
-1 & 0 & 0
\end{array}\right),
$$

como $e_{1}^{T} P=e_{3}^{T}, e_{2}^{T} P=-e_{2}^{T}$ e $e_{3}^{T} P=-e_{1}^{T}$, então existe uma permutação $\sigma \in S_{3}$ associada a $P$ e $\sigma=[321]=a_{1} a_{2} a_{1}$, dai $P \in B_{3}$. O diagrama de $\sigma \in \mathrm{S}_{3}$ é dado por

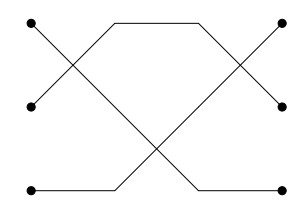

Figura 2.3: Diagrama de $\sigma=a_{1} a_{2} a_{1} \in \mathrm{S}_{3}$

Definimos a interseção de $\mathrm{B}_{n+1}$ com o grupo das matrizes ortogonais com determinante igual a 1 , como $\mathrm{B}_{n+1}^{+}=\mathrm{B}_{n+1} \cap \mathrm{SO}_{n+1}$.

Exemplo 2.2.2 A matriz do exemplo anterior

$$
P=\left(\begin{array}{ccc}
0 & 0 & 1 \\
0 & -1 & 0 \\
-1 & 0 & 0
\end{array}\right)
$$

não pertence $a \mathrm{~B}_{3}^{+}$, pois $\operatorname{det}(P)=-1$.

Entretanto, ao trocarmos um dos sinais, teremos matrizes que pertencem $a \mathrm{~B}_{3}^{+}$, por exemplo

$$
P^{\prime}=\left(\begin{array}{ccc}
0 & 0 & 1 \\
0 & -1 & 0 \\
1 & 0 & 0
\end{array}\right)
$$


onde podemos ver com facilidade que $\operatorname{det}\left(P^{\prime}\right)=1$.

Observação 2.2.1 As matrizes pertencem a grupos distintos porém representam a mesma permutação.

Definimos também o subgrupo normal $\operatorname{Diag}_{n+1}^{+} \subset \mathrm{B}_{n+1}^{+}$, que são as matrizes de permutação, diagonais e ortogonais com determinante 1. Tal subgrupo é isomorofo a $\{ \pm 1\}^{n}$.

O mapa $\phi: \mathrm{B}_{n+1}^{+} \rightarrow \mathrm{S}_{n+1}$ dado por $P \mapsto \sigma_{P}$ é um homomorfismo sobrejetor, cujo núcleo é $\operatorname{Diag}_{n+1}^{+}$. Portanto, como $\operatorname{Diag}_{n+1}^{+}$é um subgrupo normal, pelo teorema dos isomorfismos, temos que

$$
\frac{\mathrm{B}_{n+1}^{+}}{\operatorname{Diag}_{n+1}^{+}} \approx \mathrm{S}_{n+1} \text {. }
$$

Como podemos organizar os sinais em uma matriz diagonal, intuitivamente, o que esse isomorfismo nos diz é que se "esquecermos" os sinais, o que nos resulta é essencialmente uma permutação.

Assim, vimos que toda permutação $\sigma \in \mathrm{S}_{n+1}$ é associada a uma matriz $P_{\sigma} \in \mathrm{B}_{n+1}^{+}$, onde $\sigma_{P}=\sigma$. 


\section{Álgebra de Lie}

Neste capítulo, faremos uma breve revisão do conceito de álgebra de Lie, mais precisamente para o interesse do trabalho, álgebras de Lie de matrizes.

Além disso, apresentaremos a álgebra de Lie $\mathfrak{s o}_{n+1}$ do grupo $\mathrm{SO}_{n+1}$, cujo elemento $\mathfrak{a}_{i}$ é de grande importância para as construções dos próximos capítulos.

Por fim, apresentaremos o subgrupo a um parâmetro $\alpha_{i}^{\mathrm{SO}}: \mathbb{R} \rightarrow \mathrm{SO}_{n+1}$, que é definido a partir de $\mathfrak{a}_{i} \in \mathfrak{s o}_{n+1}$.

\section{1}

\section{A Álgebra de Lie}

Definição 3.1.1 Uma álgebra de Lie $\mathfrak{g}$ é um espaço vetorial sobre um corpo munido de uma operação [.,.] $: \mathfrak{g} \times \mathfrak{g} \rightarrow \mathfrak{g}$, chamada de colchete de Lie, que satisfaz as seguintes condições:

(i) Bilinearidade;

(ii) Anti-simetria, ou seja, $[X, Y]=-[Y, X]$ para todo $X, Y \in \mathfrak{g}$;

(iii) Para todo $X, Y, Z \in \mathfrak{g}$, temos $[X,[Y, Z]]+[Z,[X, Y]]+[Y,[Z, X]]=0$, esta relação é conhecida como identidade de Jacobi.

Exemplo 3.1.1 Na álgebra de Lie $\mathfrak{g l}_{n+1}$, o colchete de Lie é definido como $[A, B]=A B-B A$, para todo $A, B \in \mathfrak{g l}_{n+1}$. As condições (i) e (ii) são claras, vamos verificar a identidade de Jacobi.

Sejam $A, B, C \in \mathfrak{g l}_{n+1}$, temos

$$
\begin{aligned}
{[A,[B, C]] } & =[A, B C-C B]=A(B C-C B)-(B C-C B) A \\
& =A B C-A C B-B C A+C B A, \\
{[C,[A, B]] } & =[C, A B-B A]=C(A B-B A)-(A B-B A) C \\
& =C A B-C B A-A B C+B A C, \\
{[B,[C, A]] } & =[B, C A-A C]=B(C A-A C)-(C A-A C) B \\
& =B C A-B A C-C A B+A C B .
\end{aligned}
$$


Portanto, somando as parcelas temos

$$
\begin{gathered}
{[A,[B, C]]+[C,[A, B]]+[B,[C, A]]=A B C-A C B-B C A+C B A+} \\
C A B-C B A-A B C+B A C+B C A-B A C-C A B+A C B=0 .
\end{gathered}
$$

Logo, satisfaz a identidade de Jacobi.

Para a definição a seguir, o colchete de Lie é definido como no exemplo anterior.

Definição 3.1.2 Uma álgebra de Lie de matrizes é um espaço vetorial de matrizes, fechado pelo colchete de Lie.

Definição 3.1.3 Um grupo de Lie é um grupo que também é uma variedade real suave, de dimensão finita, em que as operações multiplicação e inverso são mapas suaves.

Definição 3.1.4 Um grupo de Lie de matrizes $G \subset \mathrm{GL}_{n+1}$ é uma variedade suave, que possui identidade e é fechada para inverso e multiplicação.

Seja $G$ um grupo de Lie de matrizes reais, como $G$ é uma superfície suave podemos tomar seu espaço tangente na identidade.

Definição 3.1.5 Defininimos o espaço tangente a $G$ na identidade $T_{\mathbf{1}}(G)$ como as matrizes na forma $X=M^{\prime}(0)$, onde $M(t)$ é um caminho suave em $G$, tal que $M(0)=\mathbf{1}$.

Proposição 3.1.1 O espaço tangente $T_{\mathbf{1}}(G)$ é fechado para o colchete de Lie, ou seja, se $X, Y \in T_{\mathbf{1}}(G)$ então $[X, Y] \in T_{\mathbf{1}}(G)$. Portanto, $T_{\mathbf{1}}(G)$ é uma álgebra de Lie de matrizes.

Prova.

Queremos $X, Y \in T_{\mathbf{1}}(G)$, para isso vamos supor $M(t)$ e $N(t)$ caminhos suaves em $\mathrm{G}$, tais que $M(0)=N(0)=\mathbf{1}, M^{\prime}(0)=X$ e $N^{\prime}(0)=Y$.

Consideremos o caminho $\Gamma_{s}(t)=M(s) N(t)(M(s))^{-1}$ para $s$ fixo. Assim, $\Gamma_{s}(t)$ é um caminho suave e $\Gamma_{s}(0)=\mathbf{1}$, daí $\Gamma_{s}^{\prime}(0) \in T_{\mathbf{1}}(G)$. Mas,

$$
\Gamma_{s}(0)=M(s) N^{\prime}(0)(M(s))^{-1}=M(s) Y(M(s))^{-1},
$$

como $M(s)$ é suave, então $\Gamma_{s}(0)$ é suave em s.

Portanto, temos um caminho suave $C(s)=\Gamma_{s}(0)$, com $C: V_{0} \rightarrow T_{\mathbf{1}}(G)$, onde $V_{0} \subset \mathbb{R}$ é uma vizinhança de 0 . Derivando o caminho, temos

$$
C^{\prime}(s)=M^{\prime}(s) Y(M(s))^{-1}-M(s) Y\left(M^{\prime}(s)\right)^{-1} M^{\prime}(s)(M(s))^{-1} .
$$


Aplicando em $s=0$, visto que $M(0)=1$ e $M^{\prime}(0)=X$, temos

$$
\begin{aligned}
C^{\prime}(0) & =M^{\prime}(0) Y(M(0))^{-1}-M(0) Y(M(0))^{-1} M^{\prime}(0)(M(0))^{-1} \\
& =X Y-Y X \\
& =[X, Y]
\end{aligned}
$$

Como a derivada de $C(s)$ aplicada em $s=0$ também está no espaço tangente $T_{\mathbf{1}}(G)$, então $[X, Y] \in T_{\mathbf{1}}(G)$.

Assim, provamos que se $X, Y \in T_{\mathbf{1}}(G)$, então $[X, Y] \in T_{\mathbf{1}}(G)$.

O assunto álgebras de Lie, mais precisamente para o nosso caso, álgebras de Lie de matrizes, é um tema de muito estudo. Contudo, não iremos nos aprofundar além deste ponto, para saber mais sobre uma boa referência é [11].

\section{2}

\section{O Grupo Ortogonal e Sua Álgebra de Lie}

O grupo especial ortogonal, denotado por

$$
\mathrm{SO}_{n+1}=\left\{M \in \mathrm{GL}_{n+1} \subset \mathbb{R}^{(n+1) \times(n+1)} \mid M M^{T}=I \quad \mathrm{e} \quad \operatorname{det}(M)=1\right\}
$$

é um grupo de Lie de matrizes, ou seja, uma superfície suave, portanto podemos tomar seu espaço tangente.

A álgebra de Lie $\mathfrak{s o}_{n+1}$, que é o espaço tangente a $\mathrm{SO}_{n+1}$ na identidade $T_{1} \mathrm{SO}_{n+1}$, consiste das matrizes reais $M \in \mathrm{SO}_{n+1}$ tais que $M+M^{T}=0$.

Assim, $\mathfrak{s o}_{n+1}=\left\{M \in \mathrm{SO}_{n+1} \mid M=-M^{T}\right\}$ são as matrizes antisimétricas. Note que a diagonal consiste de zeros, além disso as entradas abaixo da diagonal são os negativos das entradas acima, por serem anti-simétricas. Portanto, a dimensão de $\mathfrak{s o}_{n+1}$ é o número de entradas abaixo da diagonal, ou seja

$$
1+2+3+\cdots+n=\frac{(n+1) n}{2} .
$$

Como a dimensão de um grupo de Lie é a dimensão da sua álgebra, temos que a dimensão de $\mathrm{SO}_{n+1}$ é também $\frac{(n+1) n}{2}$.

Vamos então, definir alguns elementos na álgebra de Lie de $\mathrm{SO}_{n+1}$, que denotaremos por $\mathfrak{a}_{i}^{\mathrm{SO}} \in \mathfrak{s o}_{n+1}$.

Para cada $i \in \llbracket n \rrbracket$, definimos a matriz $\mathfrak{a}_{i}^{\mathrm{SO}}=e_{i+1} e_{i}^{T}-e_{i} e_{i+1}^{T}$, que é antisimétrica, ou seja, $\mathfrak{a}_{i}^{\mathrm{SO}}=-\left(\mathfrak{a}_{i}^{\mathrm{SO}}\right)^{T}$. Assim, $\mathfrak{a}_{i}^{\mathrm{SO}}$ é a matriz cujas únicas entradas não nulas são $\left(\mathfrak{a}_{i}^{\mathrm{SO}}\right)_{i+1, i}=1$ e $\left(\mathfrak{a}_{i}^{\mathrm{SO}}\right)_{i, i+1}=-1$.

A menos que seja especificado de outra forma, a partir de então, vamos considerar $\mathfrak{a}_{i}^{\mathrm{SO}}$ como $\mathfrak{a}_{i}$ para simplificar a notação. 
Exemplo 3.2.1 Se $n=3$ e $i=2$ então,

$$
\mathfrak{a}_{2}=\left(\begin{array}{cccc}
0 & 0 & 0 & 0 \\
0 & 0 & -1 & 0 \\
0 & 1 & 0 & 0 \\
0 & 0 & 0 & 0
\end{array}\right)
$$

Veremos agora o subgrupo a um parâmetro $\alpha_{i}^{\mathrm{SO}}$, definido a partir do elemento $\mathfrak{a}_{i} \in \mathfrak{s o}_{n+1}$.

Seja $\alpha_{i}^{\mathrm{SO}}: \mathbb{R} \rightarrow \mathrm{SO}_{n+1}$, dada por $\alpha_{i}^{\mathrm{SO}}(\theta)=\exp \left(\theta \mathfrak{a}_{\mathfrak{i}}\right)$. Por uma conta simples, podemos perceber que $\alpha_{i}^{\mathrm{SO}}(\theta)$ é uma matriz diagonal em blocos, dessa forma o mapa é dado por

$$
\begin{gathered}
\alpha_{i}^{\mathrm{SO}}: \mathbb{R} \rightarrow \mathrm{SO}_{n+1} \\
\alpha_{i}^{\mathrm{SO}}(\theta)=\left(\begin{array}{lll}
I_{1} & & \\
& Q(\theta) & \\
& & I_{2}
\end{array}\right),
\end{gathered}
$$

onde $I_{1} \in \mathbb{R}^{(i-1) \times(i-1)}$ e $I_{2} \in \mathbb{R}^{(n-i) \times(n-i)}$ são matrizes identidades e

$$
Q(\theta)=\left(\begin{array}{cc}
\cos (\theta) & -\sin (\theta) \\
\sin (\theta) & \cos (\theta)
\end{array}\right)
$$

Exemplo 3.2.2 Se $n=3, \theta=\pi$ e $i=2$, teremos a matriz $\mathfrak{a}_{2}$ do exemplo anterior e então

$$
\alpha_{2}^{\mathrm{SO}}(\pi)=\left(\begin{array}{cccc}
1 & 0 & 0 & 0 \\
0 & -1 & 0 & 0 \\
0 & 0 & -1 & 0 \\
0 & 0 & 0 & 1
\end{array}\right) .
$$

Exemplo 3.2.3 Se $n=4, \theta=\frac{\pi}{2}$ e $i=3$, teremos então

$$
\alpha_{3}^{\mathrm{SO}}\left(\frac{\pi}{2}\right)=\left(\begin{array}{ccccc}
1 & 0 & 0 & 0 & 0 \\
0 & 1 & 0 & 0 & 0 \\
0 & 0 & 0 & -1 & 0 \\
0 & 0 & 1 & 0 & 0 \\
0 & 0 & 0 & 0 & 1
\end{array}\right) .
$$




\section{4}

\section{Grupos de Matrizes}

Neste capítulo, estudaremos alguns exemplos de grupos de matrizes reais, que como o nome sugere, são grupos onde os elementos são matrizes reais. O nosso interesse está voltado especialmente para Quat ${ }_{n+1}$, $\operatorname{Spin}_{n+1}$ e $\tilde{\mathrm{B}}_{n+1}^{+}$.

Mais precisamente, um grupo de matrizes $G$ é um subgrupo do grupo de matrizes reais invertíveis $\mathrm{GL}_{n+1} \subset \mathbb{R}^{(n+1) \times(n+1)}$.

O grupo $\operatorname{Spin}_{n+1}$ é uma superfície suave, portanto é um grupo de Lie. Já os grupos Quat ${ }_{n+1}$ e $\tilde{\mathrm{B}}_{n+1}^{+}$, são grupos finitos. Vamos estudar tais grupos a partir da construção de seus geradores.

\section{1}

\section{O Grupo Quat ${ }_{n+1}$}

Nesta seção, definiremos o grupo Quat ${ }_{n+1}$ pelos seus geradores $\hat{a}_{i}$. Primeiramente, faremos uma construção abstrata dos geradores. Após esta definição, faremos uma construção mais concreta dos geradores como matrizes.

Definição 4.1.1 Chamamos de Quat $_{n+1}$ o grupo gerado pelos elementos $\left\{ \pm \hat{a}_{1}, \ldots, \pm \hat{a}_{n}\right\}$ que satisfazem as seguintes relaçôes:

(i) $\hat{a}_{i}^{2}=-1$;

(ii) $\hat{a}_{i} \hat{a}_{j}=\hat{a}_{j} \hat{a}_{i}$, se $|i-j| \neq 1$;

(iii) $\hat{a}_{i} \hat{a}_{j}=-\hat{a}_{j} \hat{a}_{i}$, se $|i-j|=1$.

Portanto, podemos listar seus elementos tendo assim o grupo finito

Quat $_{n+1}=\left\{ \pm 1, \pm \hat{a}_{1}, \pm \hat{a}_{2}, \pm \hat{a}_{1} \hat{a}_{2}, \pm \hat{a}_{3}, \pm \hat{a}_{1} \hat{a}_{3}, \pm \hat{a}_{2} \hat{a}_{3}, \pm \hat{a}_{1} \hat{a}_{2} \hat{a}_{3}, \ldots, \pm \hat{a}_{1} \ldots \hat{a}_{n}\right\}$

Além disso, sua cardinalidade é dada por $\mid$ Quat $_{n+1} \mid=2^{n+1}$.

O grupo Quat ${ }_{n+1}$ pode ser visto como um grupo de matrizes reais $2^{n} \times 2^{n}$, basta interpretarmos seus geradores como matrizes.

Antes de descrevermos a forma geral de um gerador $\hat{a}_{i}$ para qualquer $n \geq 1$ e $i \in \llbracket n \rrbracket$, vamos ver a construção do primeiro caso.

Se $n=1$, então $\hat{a}_{1}$ é o único gerador e é uma matriz $2 \times 2$ satisfazendo as condições acima. 
Dessa forma, é fácil ver que

$$
\hat{a}_{1}=\left(\begin{array}{cc}
0 & -1 \\
1 & 0
\end{array}\right) .
$$

Para o caso geral, quando $i=1$ temos que a matriz $\hat{a}_{1}$ é uma matriz diagonal em blocos, onde cada bloco $J$ é de dimensão $2 \times 2$ dado como

$$
J=\left(\begin{array}{cc}
0 & -1 \\
1 & 0
\end{array}\right) .
$$

Portanto, a matriz $\hat{a}_{1}$ é uma matriz com $2^{n-1}$ blocos $J$ na diagonal, ou seja,

$$
\hat{a}_{1}=\left(\begin{array}{ccc}
J & & \\
& \ddots & \\
& & J
\end{array}\right) .
$$

Vamos generalizar a matriz $\hat{a}_{i}$ para todo $n \geq 1$ e $i \geq 2$. A construção é parecida com o caso $i=1$. A matriz é em blocos na sua diagonal, onde cada bloco tem dimensão $2^{i} \times 2^{i}$ e é da forma:

$$
J=\left(\begin{array}{cccc} 
& & -I & \\
& & & I \\
I & & & \\
& & &
\end{array}\right), \quad \operatorname{com} I \in \mathbb{R}^{\left(2^{i-2}\right) \times\left(2^{i-2}\right)} .
$$

Portanto, $\hat{a}_{i}$ é uma matriz com $2^{n-i}$ blocos $J$ na diagonal, ou seja,

$$
\hat{a}_{i}=\left(\begin{array}{ccc}
J & & \\
& \ddots & \\
& & J
\end{array}\right) .
$$

Observação 4.1.1 Se $i=2$, então cada matriz de dimensão $2^{i-2} \times 2^{i-2}$ é apenas uma entrada.

Agora que construímos as matrizes $\hat{a}_{i}$, vamos verificar que elas satisfazem as condições da definição.

Como $\hat{a}_{i}$ é uma matriz diagonal em blocos, então $\hat{a}_{i}^{2}$ é da mesma forma 
e os blocos são os quadrados dos blocos originais, daí

$$
\hat{a}_{i}^{2}=\left(\begin{array}{ccc}
J & & \\
& \ddots & \\
& & J
\end{array}\right) \cdot\left(\begin{array}{lll}
J & & \\
& \ddots & \\
& & J
\end{array}\right)=\left(\begin{array}{lll}
J^{2} & & \\
& \ddots & \\
& & J^{2}
\end{array}\right)
$$

Mas $J$ é uma matriz em dois blocos, vamos denotar o de cima por $A$ e o de baixo por $-A$, então para calcular $J^{2}$ basta tomarmos o produto dos seus blocos da seguinte forma

$$
\begin{gathered}
J^{2}=\left(\begin{array}{rr} 
& A \\
-A &
\end{array}\right) \cdot\left(\begin{array}{rr} 
& A \\
-A &
\end{array}\right)=\left(\begin{array}{ll}
A \cdot(-A) & \\
& (-A) \cdot A
\end{array}\right), \text { onde } \\
A=\left(\begin{array}{ll}
-I & \\
& I
\end{array}\right) .
\end{gathered}
$$

Como $A$ e $-A$ comutam, basta verificarmos que $A \cdot(-A)=(-A) \cdot A=-I$, mas $A$ e $-A$ são matrizes diagonais em blocos de mesma dimensão, portanto basta fazer o produto dos blocos, daí

$$
(-A) \cdot A=A \cdot(-A)=\left(\begin{array}{ll}
-I & \\
& I
\end{array}\right) \cdot\left(\begin{array}{ll}
I & \\
& -I
\end{array}\right)=\left(\begin{array}{ll}
-I & \\
& -I
\end{array}\right) .
$$

Agora que sabemos exatamente quem é $J^{2}$, temos que

$$
\hat{a}_{i}^{2}=\left(\begin{array}{ccc}
J^{2} & & \\
& \ddots & \\
& & J^{2}
\end{array}\right)=\left(\begin{array}{ccc}
-1 & & \\
& \ddots & \\
& & -1
\end{array}\right)
$$

portanto as matrizes $\hat{a}_{i}$ satisfazem a primeira condição da definição, $\hat{a}_{i}^{2}=-1$, onde nesse caso, $-1=-I$.

Falta verificarmos as outras duas condições. Primeiramente faremos o caso $|i-j|=1$.

Para facilitar um pouco as contas, vamos considerar as matrizes em 4 grandes blocos, o que pode ser feito já que as dimensões das matrizes são múltiplas de 4 , para os casos $n \geq 2$.

Como $\hat{a}_{1}$ é um caso distinto, faremos primeiro $\hat{a}_{1} \hat{a}_{2}=-\hat{a}_{2} \hat{a}_{1}$, que é a única possibilidade onde aparece $\hat{a}_{1}$, no caso $|i-j|=1$. Assim, $\hat{a}_{1}$ é em blocos 
$2 \times 2$ e $\hat{a}_{2}$ em blocos $4 \times 4$. Portanto, em blocos $4 \times 4$, temos

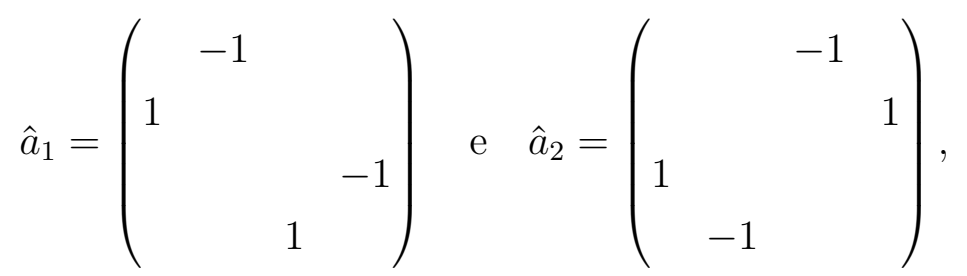

fazendo os produtos $\hat{a}_{1} \hat{a}_{2}$ e $\hat{a}_{2} \hat{a}_{1}$ temos, respectivamente

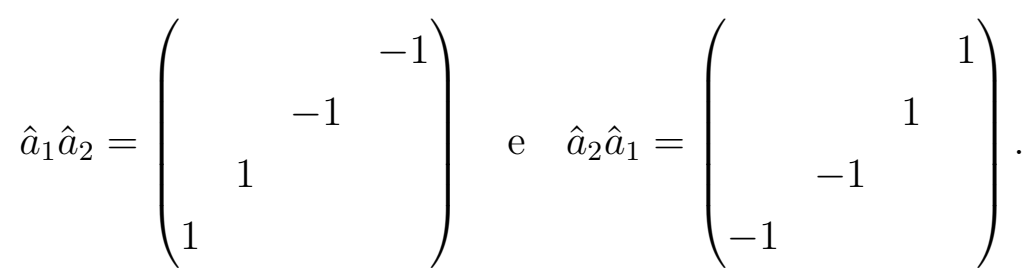

Portanto, $\hat{a}_{1} \hat{a}_{2}=-\hat{a}_{2} \hat{a}_{1}$.

Para o caso geral, note que supondo $j=i+1$, cada matriz $J$ em $\hat{a}_{j}$ tem metade da dimensão de $\hat{a}_{i}$. Suponha por exemplo, $i=2$ e $j=3$ então, $J$ de $\hat{a}_{2}$ tem dimensão $2^{n-2}$ e $J$ de $\hat{a}_{3}$ tem dimensão $2^{n-3}=\frac{2^{n-2}}{2}$.

Dessa forma, as matrizes $\hat{a}_{i}$ e $\hat{a}_{j}$, onde nesse caso $I \in \mathbb{R}^{\left(2^{i-2}\right) \times\left(2^{i-2}\right)}$, são

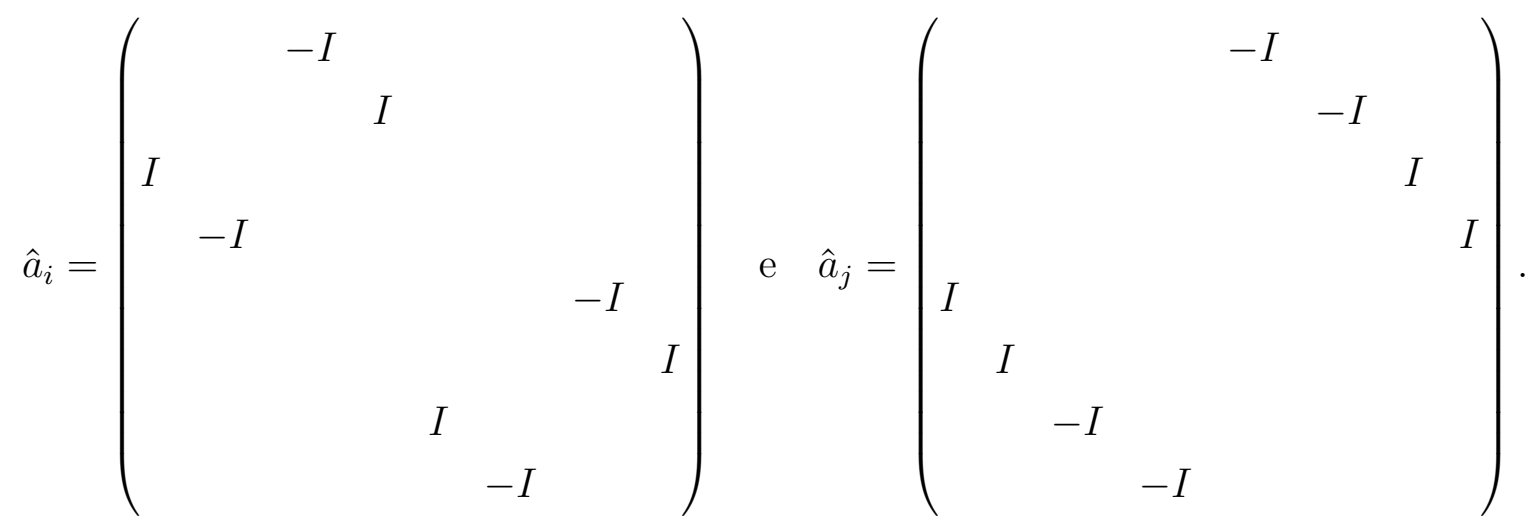

Portanto, fazendo os produtos $\hat{a}_{i} \hat{a}_{j}$ e $\hat{a}_{j} \hat{a}_{i}$ obtemos, respectivamente

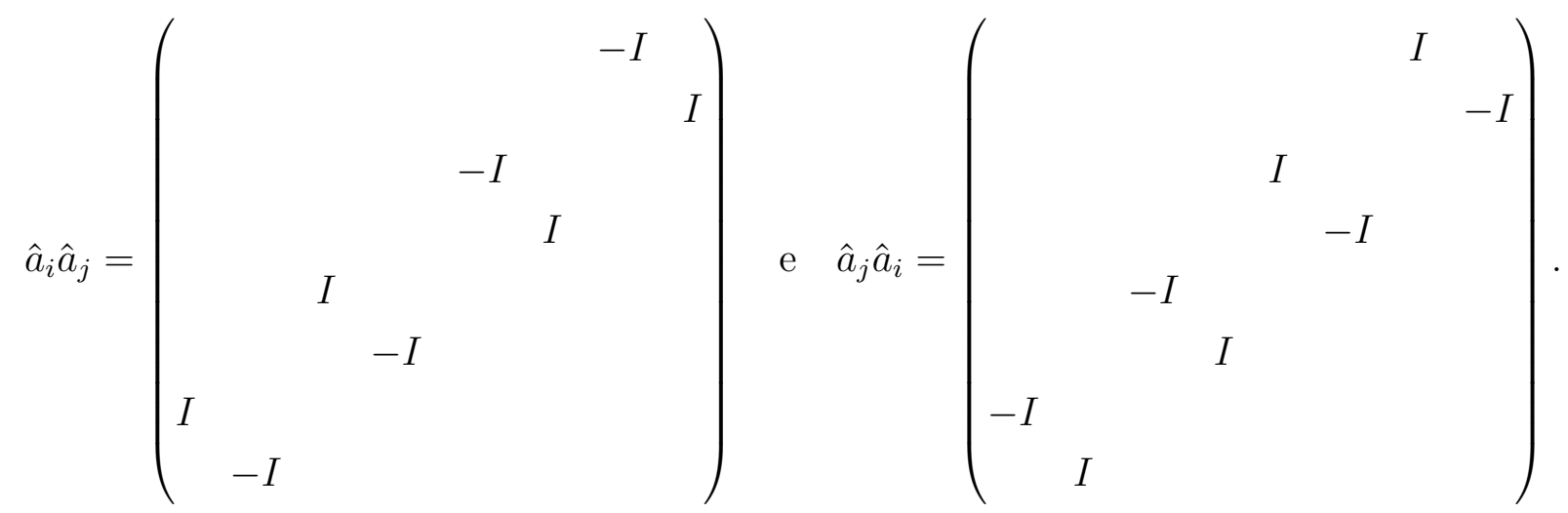

Assim, mostramos que se $|i-j|=1$, então $\hat{a}_{i} \hat{a}_{j}=-\hat{a}_{j} \hat{a}_{i}$. 
Por fim, precisamos verificar que se $|i-j| \neq 1$, então $\hat{a}_{i} \hat{a}_{j}=\hat{a}_{j} \hat{a}_{i}$. Primeiro faremos o caso $i=1$ e $j \geq 3$.

Vamos escrever $\hat{a}_{1}$ em blocos $J_{1}$, onde a quatidade de blocos que formam $J_{1}$ é um múltiplo de 4 , da seguinte forma

$$
\hat{a}_{1}=\left(\begin{array}{cccc}
J_{1} & & & \\
& J_{1} & & \\
& & J_{1} & \\
& & & J_{1}
\end{array}\right), \quad \text { onde } \quad J_{1}=\left(\begin{array}{cccc}
y^{-1} & & \\
& & & \\
& & \ddots & \\
& & & -1 \\
& & & 1
\end{array}\right)
$$

Note que no menor caso, onde $j=3$, temos que $I$ é uma matriz $2 \times 2$, a mesma dimensão de cada bloco que forma a matriz $J_{1}$. Para o caso $j \geq 3$, a dimensão de cada bloco $I$ é um múltiplo da dimensão dos blocos que formam $J_{1}$. Portanto,

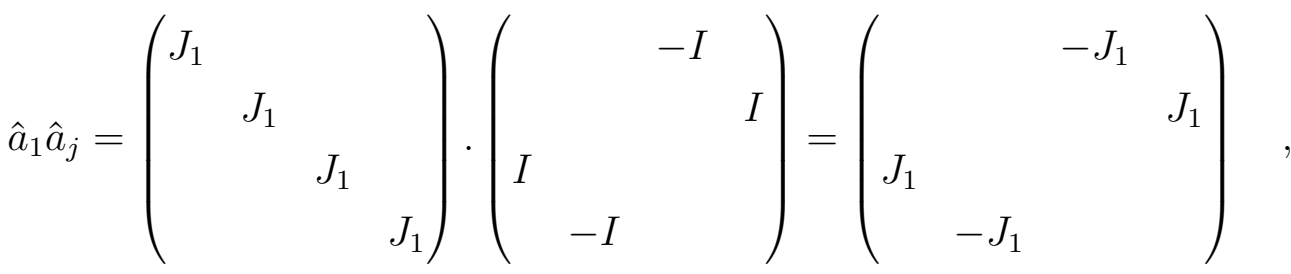

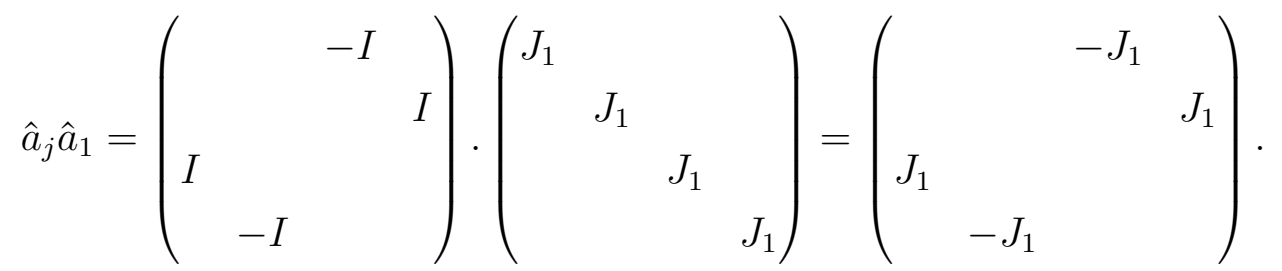

Assim, $\hat{a}_{1} \hat{a}_{j}=\hat{a}_{j} \hat{a}_{1}$.

Para o caso geral $i \geq 2$ e $j \geq i+2$ a construção é similar, a diferença é que $\hat{a}_{i}$ é escrita como na forma geral

$$
\hat{a}_{i}=\left(\begin{array}{ccc}
J & & \\
& \ddots & \\
& & J
\end{array}\right), \quad \text { onde } \quad J=\left(\begin{array}{ccc} 
& -I \\
& & \\
I & & \\
& -I &
\end{array}\right)
$$

dessa forma, o produto fica fácil de ser calculado, pois cada bloco $J$ é da mesma 
forma de $\hat{a}_{j}$, portanto

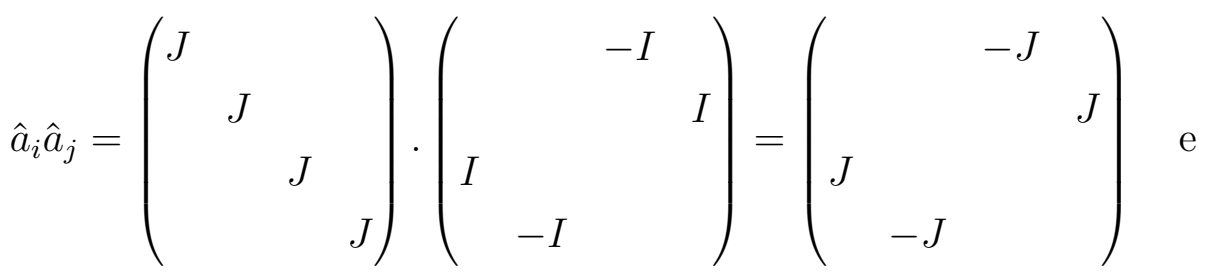

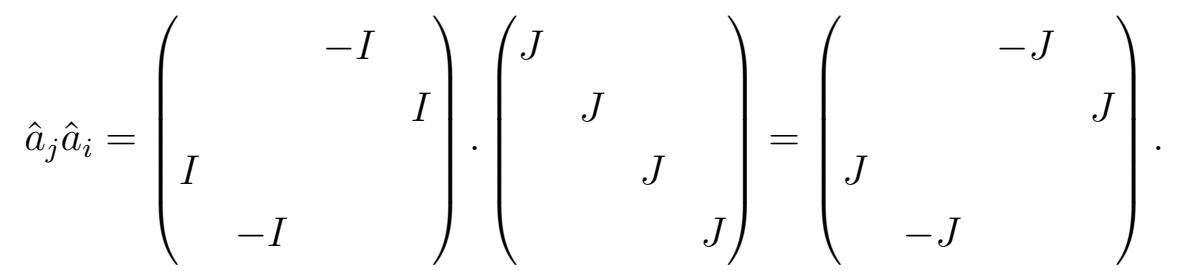

$\operatorname{Assim} \hat{a}_{i} \hat{a}_{j}=\hat{a}_{j} \hat{a}_{i}$.

Com a prova desta igualdade, mostramos que as matrizes $\hat{a}_{i}$ que construímos, satisfazem as condições da definição dos geradores do grupo Quat ${ }_{n+1}$. Portanto, podemos considerá-lo como um grupo de matrizes finito.

Observe que cada $\hat{a}_{i}$ é uma matriz anti-simétrica. Note também, que cada bloco que forma a matriz $\hat{a}_{i}$ tem determinante 1 , visto que $J$ é também uma matriz em blocos, onde cada bloco não nulo tem determinante 1 . Assim, $\hat{a}_{i}$ tem determinante 1.

Com isso, como cada elemento $q \in$ Quat $_{n+1}$ é um produto dos geradores $\hat{a}_{i}$, ou seja

$$
q= \pm \hat{a}_{1}^{\varepsilon_{1}} \ldots \hat{a}_{n}^{\varepsilon_{n}} \in \text { Quat }_{n+1}
$$

com $\varepsilon_{k} \in\{0,1\}$, temos que $\operatorname{det}(q)=1$.

As matrizes $\hat{a}_{i}$ portanto, tem em cada coluna uma única entrada não nula, tal entrada possui valor 1 ou -1 . Além disso, sabemos que $\operatorname{det} \hat{a}_{i}=1$. Assim, $\hat{a}_{i}$ é uma matriz de permutação com sinal, ou seja, $\hat{a}_{i} \in \mathrm{B}_{2^{n}}^{+}$para todo $i \in \llbracket n \rrbracket$.

Exemplo 4.1.1 Se $n=2$, então para $i \in\{1,2\}$, $\hat{a}_{i}$ são matrizes $2^{2} \times 2^{2} d a$ seguinte forma:

$$
\hat{a}_{1}=\left(\begin{array}{cccc}
0 & -1 & 0 & 0 \\
1 & 0 & 0 & 0 \\
0 & 0 & 0 & -1 \\
0 & 0 & 1 & 0
\end{array}\right) \quad \text { e } \quad \hat{a}_{2}=\left(\begin{array}{cccc}
0 & 0 & -1 & 0 \\
0 & 0 & 0 & 1 \\
1 & 0 & 0 & 0 \\
0 & -1 & 0 & 0
\end{array}\right)
$$

Exemplo 4.1.2 Se $n=3$, então $\hat{a}_{i}$ com $i \in\{1,2,3\}$ são matrizes $8 \times 8$. Pelo exemplo anterior, sabemos quem são $\hat{a}_{1}$ e $\hat{a}_{2}$ no caso $n=2$. Portanto, para $n=3$ 
basta repetirmos as mesmas matrizes diagonalmente, ou seja,

$$
\hat{a}_{1}=\left(\begin{array}{cccccccc}
0 & -1 & & & & & & \\
1 & 0 & & & & & & \\
& & 0 & -1 & & & & \\
& & 0 & & & & \\
& & & 0 & -1 & & \\
& & & 1 & 0 & & \\
& & & & & 0 & -1 \\
& & & & & 1 & 0
\end{array}\right), \hat{a}_{2}=\left(\begin{array}{cccccccc}
0 & 0 & -1 & 0 & & & & \\
0 & 0 & 0 & 1 & & & & \\
1 & 0 & 0 & 0 & & & & \\
0 & -1 & 0 & 0 & & & & \\
& & & & 0 & 0 & -1 & 0 \\
& & & & 0 & 0 & 0 & 1 \\
& & & & 1 & 0 & 0 & 0 \\
& & & & 0 & -1 & 0 & 0
\end{array}\right) .
$$

Já para o caso $i=3$, temos

$$
\hat{a}_{3}=\left(\begin{array}{cccccccc}
0 & 0 & 0 & 0 & -1 & 0 & 0 & 0 \\
0 & 0 & 0 & 0 & 0 & -1 & 0 & 0 \\
0 & 0 & 0 & 0 & 0 & 0 & 1 & 0 \\
0 & 0 & 0 & 0 & 0 & 0 & 0 & 1 \\
1 & 0 & 0 & 0 & 0 & 0 & 0 & 0 \\
0 & 1 & 0 & 0 & 0 & 0 & 0 & 0 \\
0 & 0 & -1 & 0 & 0 & 0 & 0 & 0 \\
0 & 0 & 0 & -1 & 0 & 0 & 0 & 0
\end{array}\right)
$$

A álgebra de matrizes gerada pelos elementos $\hat{a}_{i}$ é chamada álgebra de Clifford, denotada por $\mathrm{Cl}_{n+1}^{0}$. Na próxima seção veremos esta álgebra com mais detalhes.

\section{2}

\section{A Álgebra de Clifford}

Na seção anterior, vimos que Quat $_{n+1}$ é um grupo finito e que seus elementos são precisamente

Quat $_{n+1}=\left\{ \pm 1, \pm \hat{a}_{1}, \pm \hat{a}_{2}, \pm \hat{a}_{1} \hat{a}_{2}, \pm \hat{a}_{3}, \pm \hat{a}_{1} \hat{a}_{3}, \pm \hat{a}_{2} \hat{a}_{3}, \pm \hat{a}_{1} \hat{a}_{2} \hat{a}_{3}, \ldots, \pm \hat{a}_{1} \ldots \hat{a}_{n}\right\}$.

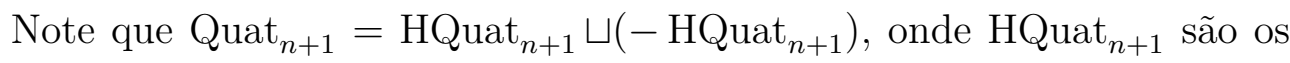
elementos que aparecem com sinal + em $^{\text {Quat }}{ }_{n+1}$. Além disso, observe que HQuat $_{n+1} \subset$ Quat $_{n+1}$ não é um subgrupo, visto que $\hat{a}_{i}^{2}=-1 \notin$ HQuat $_{n+1}$.

Definição 4.2.1 $\mathrm{Cl}_{n+1}^{0}$ é uma álgebra associativa com unidade sobre $\mathbb{R}$, que é um espaço vetorial de dimensão $2^{n}$, com base ortonormal HQuat ${ }_{n+1}$. 
Portanto, a álgebra de Clifford $\mathrm{Cl}_{n+1}^{0}$ é gerada pelos elementos $\hat{a}_{i}$, que satisfazem as relações já vistas anteriormente na definição dos geradores de Quat $_{n+1}$. Recorde:

(i) $\hat{a}_{i}^{2}=-1$;

(ii) $\hat{a}_{i} \hat{a}_{j}=\hat{a}_{j} \hat{a}_{i}$, se $|i-j| \neq 1$;

(iii) $\hat{a}_{i} \hat{a}_{j}=-\hat{a}_{j} \hat{a}_{i}$, se $|i-j|=1$.

Além disso, como espaço vetorial é dotada de um produto interno, definido por

$$
\left\langle z_{1}, z_{2}\right\rangle=2^{-n} \operatorname{Traço}\left(z_{1} z_{2}^{T}\right)
$$

Quando $n$ é pequeno o suficiente, as álgebras de Clifford são álgebras conhecidas. Vamos ver alguns exemplos.

Exemplo 4.2.1 Se $n=0$, então $\mathrm{Cl}_{1}^{0}$ é uma álgebra de dimensão 1 sobre $\mathbb{R}$, tal que sua base é $\{1\}$. Ou seja, a álgebra de Clifford $\mathrm{Cl}_{1}^{0}$ é o próprio $\mathbb{R}$.

Exemplo 4.2.2 Se $n=1$, então $\mathrm{Cl}_{2}^{0}$ é uma álgebra de dimensão 2 sobre $\mathbb{R}$ cuja base é $\left\{1, \hat{a}_{1}\right\}$, que são matrizes $2 \times 2$ satisfazendo a condição $\hat{a}_{1}^{2}=-1$.

Portanto, os elementos de $\mathrm{Cl}_{2}^{0}$ são da forma $u+v \hat{a}_{1}$, onde $u, v \in \mathbb{R}$. Sabemos da seção anterior, que

$$
\hat{a}_{1}=\left(\begin{array}{cc}
0 & -1 \\
1 & 0
\end{array}\right) .
$$

Note que esta é a forma matricial de $\mathbf{i} \in \mathbb{C}$. Portanto, os elementos de $\mathrm{Cl}_{2}^{0}$ são dados por $u+$ va $\hat{a}_{1}$, onde $\hat{a}_{1}=\mathbf{i}$, ou seja, $\mathrm{Cl}_{2}^{0}=\mathbb{C}$.

Antes de vermos mais um exemplo de álgebra de Clifford, vamos nos recordar dos quatérnios $\mathbb{H}$.

O conjunto dos quatérnios é um espaço vetorial sobre $\mathbb{R}$, com dimensão 4, cuja multiplicação é associativa e distributiva com a soma. Além disso, com exceção da multiplicação por escalar, não é comutativa. Portanto, $\mathbb{H}$ é uma álgebra associativa e não comutativa.

Um elemento $q \in \mathbb{H}$ é tal que $q=u \mathbf{1}+v \mathbf{i}+w \mathbf{j}+t \mathbf{k}$, onde $u, v, w, t \in \mathbb{R}$ cuja norma é $|q|=\sqrt{u^{2}+v^{2}+w^{2}+t^{2}}$. Podemos associar $q \in \mathbb{H}$ a matriz

$$
q=\left(\begin{array}{cccc}
u & -v & -w & -t \\
v & u & -t & w \\
w & t & u & -v \\
t & -w & v & u
\end{array}\right)
$$


onde, 1 é a matriz identidade $4 \times 4 \mathrm{e}$

$$
\mathbf{i}=\left(\begin{array}{cccc}
0 & -1 & 0 & 0 \\
1 & 0 & 0 & 0 \\
0 & 0 & 0 & -1 \\
0 & 0 & 1 & 0
\end{array}\right), \quad \mathbf{j}=\left(\begin{array}{cccc}
0 & 0 & -1 & 0 \\
0 & 0 & 0 & 1 \\
1 & 0 & 0 & 0 \\
0 & -1 & 0 & 0
\end{array}\right), \quad \mathbf{k}=\left(\begin{array}{cccc}
0 & 0 & 0 & -1 \\
0 & 0 & -1 & 0 \\
0 & 1 & 0 & 0 \\
1 & 0 & 0 & 0
\end{array}\right)
$$

Os elementos $\mathbf{i}, \mathbf{j}$ e $\mathbf{k}$ são tais que $\mathbf{i}^{2}=\mathbf{j}^{2}=\mathbf{k}^{2}=-\mathbf{1}$, além disso $-\mathbf{j i}=\mathbf{i j}=\mathbf{k},-\mathbf{k j}=\mathbf{j k}=\mathbf{i}$ e $-\mathbf{i k}=\mathbf{k i}=\mathbf{j}$.

Como o produto de dois elementos $\mathbf{i}, \mathbf{j}$ e $\mathbf{k}$ é mais ou menos o outro, então $\{ \pm 1, \pm \mathbf{i}, \pm \mathbf{j}, \pm \mathbf{k}\}$ é um grupo não abeliano. Denota-se tal grupo como $Q_{8}$.

Exemplo 4.2.3 Se $n=2$, então $\mathrm{Cl}_{3}^{0}$ é uma álgebra de dimensão 4 sobre $\mathbb{R}$, cuja base é $\left\{1, \hat{a}_{1}, \hat{a}_{2}, \hat{a}_{1} \hat{a}_{2}\right\}$, que são matrizes $4 \times 4$ satisfazendo as condições (i), (ii) e (iii) da definição, ou seja,

$$
\hat{a}_{1}^{2}=-1=\hat{a}_{2}^{2}, \quad \hat{a}_{1} \hat{a}_{2}=-\hat{a}_{2} \hat{a}_{1}
$$

Assim, como vimos anteriormente, essa é exatamente a álgebra dos quatérnios $\mathbb{H}$, basta tomarmos

$$
\hat{a}_{1}=\mathbf{i}, \hat{a}_{2}=\mathbf{j}, \hat{a}_{1} \hat{a}_{2}=\mathbf{k} \text {. }
$$

Com isso, já conhecemos três exemplos de álgebras de Clifford: $\mathrm{Cl}_{1}^{0}=\mathbb{R}$, $\mathrm{Cl}_{2}^{0}=\mathbb{C}, \mathrm{Cl}_{3}^{0}=\mathbb{H}$.

Como $2^{n}$ cresce muito rápido, descrever os geradores das álgebras de Clifford para $n \geq 2$, passa a ser uma tarefa relativamente trabalhosa e extensa.

Classificações para as álgebras de Clifford podem ser encontradas em [12].

\section{3}

\section{O Subgrupo a Um Parâmetro $\alpha_{i}^{\text {Spin }}$}

Um subgrupo a um parâmentro de um grupo G é um homomorfismo contínuo de grupos de $\mathbb{R}$, como grupo aditivo, no grupo $\mathrm{G}$ em questão.

A partir dos geradores $\hat{a}_{i} \in$ Quat $_{n+1}$, vamos definir os subgrupos a um parâmetro do grupo $\mathrm{SO}_{2^{n}}$

$$
\begin{gathered}
\alpha_{i}^{\text {Spin }}: \mathbb{R} \rightarrow \mathrm{SO}_{2^{n}} \\
\alpha_{i}^{\mathrm{Spin}}(\theta) \mapsto \exp \left(\theta \frac{\hat{a}_{i}}{2}\right) .
\end{gathered}
$$


Como $\hat{a}_{i}$ são matrizes com diagonal nula, não é difícil ver que $\exp \left(\theta \frac{\hat{a}_{i}}{2}\right)$ é uma matriz cujas entradas diagonais são $\cos \left(\frac{\theta}{2}\right)$, e nas posições das entradas não nulas de $\hat{a}_{i}$, temos $\sin \left(\frac{\theta}{2}\right)$.

Portanto, os elementos $\alpha_{i}^{\text {Spin }}(\theta)$ são matrizes $2^{n} \times 2^{n}$ definidas como

$$
\alpha_{i}^{\operatorname{Spin}}(\theta)=\exp \left(\theta \frac{\hat{a}_{i}}{2}\right)=\cos \left(\frac{\theta}{2}\right)+\hat{a}_{i} \sin \left(\frac{\theta}{2}\right)
$$

Notação 4.3.1 Para simplificar a notação, trataremos $\alpha_{i}^{\mathrm{Spin}}(\theta) \operatorname{como} \alpha_{i}(\theta)$, a menos que seja especificado de outra forma.

Vamos então, escrever tais elementos como matrizes. Para que fique mais claro, antes de formalizarmos a forma matricial de $\alpha_{i}(\theta)$, construiremos os $\operatorname{casos} n=2$ e $n=3$.

Observação 4.3.1 Como $\cos \left(\frac{\pi}{2}\right)=0 e \sin \left(\frac{\pi}{2}\right)=1, \alpha_{i}(\pi)=\hat{a}_{i} \in$ Quat $_{n+1}$.

Exemplo 4.3.1 Se $n=2$ e $i=1$, temos

$$
\alpha_{1}(\theta)=\left(\begin{array}{cccc}
\cos \left(\frac{\theta}{2}\right) & -\sin \left(\frac{\theta}{2}\right) & 0 & 0 \\
\sin \left(\frac{\theta}{2}\right) & \cos \left(\frac{\theta}{2}\right) & 0 & 0 \\
0 & 0 & \cos \left(\frac{\theta}{2}\right) & -\sin \left(\frac{\theta}{2}\right) \\
0 & 0 & \sin \left(\frac{\theta}{2}\right) & \cos \left(\frac{\theta}{2}\right)
\end{array}\right)
$$

Se $i=2$, temos

$$
\alpha_{2}(\theta)=\left(\begin{array}{cccc}
\cos \left(\frac{\theta}{2}\right) & 0 & -\sin \left(\frac{\theta}{2}\right) & 0 \\
0 & \cos \left(\frac{\theta}{2}\right) & 0 & \sin \left(\frac{\theta}{2}\right) \\
\sin \left(\frac{\theta}{2}\right) & 0 & \cos \left(\frac{\theta}{2}\right) & 0 \\
0 & -\sin \left(\frac{\theta}{2}\right) & 0 & \cos \left(\frac{\theta}{2}\right)
\end{array}\right)
$$

Note que $\operatorname{det}\left(\alpha_{i}(\theta)\right)=\left(\cos \left(\frac{\theta}{2}\right)^{2}+\sin \left(\frac{\theta}{2}\right)^{2}\right)^{2}=1$, para $i \in\{1,2\}$.

Exemplo 4.3.2 Se $n=3$ e $i=1, \alpha_{1}(\theta)$ tem dimensão $8 \times 8$ e é dada por:

$$
\alpha_{1}(\theta)=\left(\begin{array}{llll}
Q(\theta) & & & \\
& Q(\theta) & & \\
& & Q(\theta) & \\
& & & Q(\theta)
\end{array}\right)
$$

onde

$$
Q(\theta)=\left(\begin{array}{cc}
\cos \left(\frac{\theta}{2}\right) & -\sin \left(\frac{\theta}{2}\right) \\
\sin \left(\frac{\theta}{2}\right) & \cos \left(\frac{\theta}{2}\right)
\end{array}\right)
$$


Quando $i=2$, a matriz $\alpha_{2}(\theta)$ é

$$
\alpha_{2}(\theta)=\left(\begin{array}{cccccccc}
\cos \left(\frac{\theta}{2}\right) & 0 & -\sin \left(\frac{\theta}{2}\right) & 0 & 0 & 0 & 0 & 0 \\
0 & \cos \left(\frac{\theta}{2}\right) & 0 & \sin \left(\frac{\theta}{2}\right) & 0 & 0 & 0 & 0 \\
\sin \left(\frac{\theta}{2}\right) & 0 & \cos \left(\frac{\theta}{2}\right) & 0 & 0 & 0 & 0 & 0 \\
0 & -\sin \left(\frac{\theta}{2}\right) & 0 & \cos \left(\frac{\theta}{2}\right) & 0 & 0 & 0 & 0 \\
0 & 0 & 0 & 0 & \cos \left(\frac{\theta}{2}\right) & 0 & -\sin \left(\frac{\theta}{2}\right) & 0 \\
0 & 0 & 0 & 0 & 0 & \cos \left(\frac{\theta}{2}\right) & 0 & \sin \left(\frac{\theta}{2}\right) \\
0 & 0 & 0 & 0 & \sin \left(\frac{\theta}{2}\right) & 0 & \cos \left(\frac{\theta}{2}\right) & 0 \\
0 & 0 & 0 & 0 & 0 & -\sin \left(\frac{\theta}{2}\right) & 0 & \cos \left(\frac{\theta}{2}\right)
\end{array}\right) .
$$

Por fim, quando $i=3 \alpha_{3}(\theta)$ é uma matriz de um único bloco $8 \times 8$

\begin{tabular}{|c|c|c|c|c|c|c|c|c|}
\hline \multirow{8}{*}{$\alpha_{3}(\theta)=$} & $\left(\cos \left(\frac{\theta}{2}\right)\right.$ & 0 & 0 & 0 & $-\sin \left(\frac{\theta}{2}\right)$ & 0 & 0 & 0 \\
\hline & 0 & $\cos \left(\frac{\theta}{2}\right)$ & 0 & 0 & 0 & $-\sin \left(\frac{\theta}{2}\right)$ & 0 & 0 \\
\hline & 0 & 0 & $\cos \left(\frac{\theta}{2}\right)$ & 0 & 0 & 0 & $\sin \left(\frac{\theta}{2}\right)$ & 0 \\
\hline & 0 & 0 & 0 & $\cos \left(\frac{\theta}{2}\right)$ & 0 & 0 & 0 & $\sin \left(\frac{\theta}{2}\right)$ \\
\hline & $\sin \left(\frac{\theta}{2}\right)$ & 0 & 0 & 0 & $\cos \left(\frac{\theta}{2}\right)$ & 0 & 0 & 0 \\
\hline & 0 & $\sin \left(\frac{\theta}{2}\right)$ & 0 & 0 & 0 & $\cos \left(\frac{\theta}{2}\right)$ & 0 & 0 \\
\hline & 0 & 0 & $-\sin \left(\frac{\theta}{2}\right)$ & 0 & 0 & 0 & $\cos \left(\frac{\theta}{2}\right)$ & 0 \\
\hline & 0 & 0 & 0 & $-\sin \left(\frac{\theta}{2}\right)$ & 0 & 0 & 0 & $\cos \left(\frac{\theta}{2}\right)$ \\
\hline
\end{tabular}

Note que $\operatorname{det}\left(\alpha_{i}(\theta)\right)=\left(\cos \left(\frac{\theta}{2}\right)^{2}+\sin \left(\frac{\theta}{2}\right)^{2}\right)^{4}=1$, para $i \in\{1,2,3\}$.

Agora descreveremos a forma geral, primeiramente para $i=1$, em seguida para $i \geq 2$.

Quando $i=1$, temos $\alpha_{1}(\theta)=\exp \left(\theta \frac{\hat{a}_{1}}{2}\right)=\cos \left(\frac{\theta}{2}\right)+\hat{a}_{1} \sin \left(\frac{\theta}{2}\right)$. Como visto anteriormente,

$$
\hat{a}_{1}=\left(\begin{array}{ccccc}
0 & -1 & & & \\
1 & 0 & & & \\
& & \ddots & & \\
& & & 0 & -1 \\
& & & 1 & 0
\end{array}\right),
$$


portanto $\alpha_{1}(\theta)=\cos \left(\frac{\theta}{2}\right)+\hat{a}_{1} \sin \left(\frac{\theta}{2}\right)$ é dada por

$\alpha_{1}(\theta)=\left(\begin{array}{ccc}\cos \left(\frac{\theta}{2}\right) & & \\ & \ddots & \\ & & \cos \left(\frac{\theta}{2}\right)\end{array}\right)+\left(\begin{array}{ccccc}0 & -1 & & & \\ 1 & 0 & & & \\ & & \ddots & & \\ & & & 0 & -1 \\ & & & 1 & 0\end{array}\right) \cdot\left(\begin{array}{ccc}\sin \left(\frac{\theta}{2}\right) & & \\ & \ddots & \\ & & \sin \left(\frac{\theta}{2}\right)\end{array}\right)$

Logo, temos que a matriz $\alpha_{1}(\theta)$ é uma matriz em blocos na sua diagonal, assim como no exemplo anterior, dada por

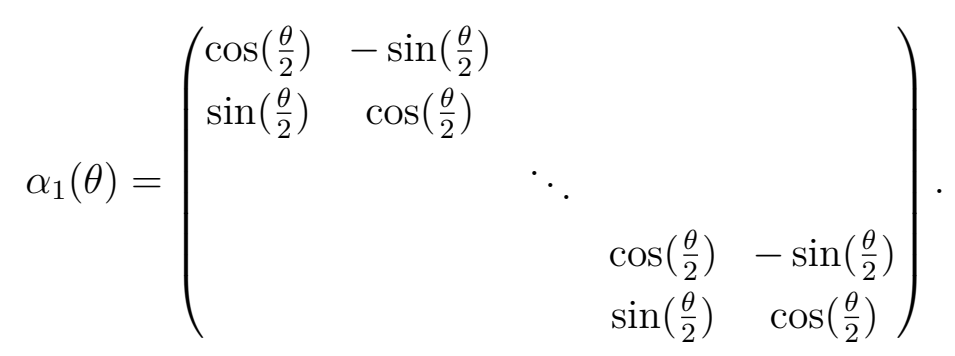

Descreveremos agora, a forma geral para $\alpha_{i}(\theta)$ para todo $i \geq 2$, que é uma matriz diagonal em blocos iguais, onde cada bloco tem dimensão $2^{i} \times 2^{i}$ e é definido como

$$
Q_{i}(\theta)=\left(\begin{array}{cccc}
C(\theta) & & -S(\theta) & \\
& C(\theta) & & S(\theta) \\
S(\theta) & & C(\theta) & \\
& -S(\theta) & & C(\theta)
\end{array}\right)
$$

onde cada matriz $C(\theta)$ e $S(\theta)$ tem dimensão $2^{i-2} \times 2^{i-2}$ e é diagonal de cossenos e senos, respectivamente, ou seja,

$$
C(\theta)=\left(\begin{array}{ccc}
\cos \left(\frac{\theta}{2}\right) & & \\
& \ddots & \\
& & \cos \left(\frac{\theta}{2}\right)
\end{array}\right) \text { e } S(\theta)=\left(\begin{array}{lll}
\sin \left(\frac{\theta}{2}\right) & & \\
& \ddots & \\
& & \sin \left(\frac{\theta}{2}\right)
\end{array}\right)
$$

Portanto, para todo $i \geq 2$ a matriz $\alpha_{i}(\theta)$ é dada por

$$
\alpha_{i}(\theta)=\left(\begin{array}{ccc}
Q_{i} & & \\
& \ddots & \\
& & Q_{i}
\end{array}\right)
$$

Observação 4.3.2 Ao voltar nos exemplos anteriores, pode-se verificar a forma geral para os casos $n=2$ e $n=3$. 
Assim como Quat $_{n+1}$, o grupo gerado pelos elementos $\alpha_{i}(\theta)$, pode ser pensado de forma abstrata e também matricial.

Note que os elementos $\alpha_{i}(\theta)$ são matrizes ortogonais em blocos, cujos elementos da diagonal são iguais em cada matriz. Além disso, o determinante de cada bloco é 1 , portanto o determinante da matriz toda é 1 .

\section{4}

\section{O Grupo $\operatorname{Spin}_{n+1}$}

Tendo definidos $\alpha_{i}(\theta)$, vamos agora considerar o grupo que tais elementos geram.

Lembre que o subgrupo a um parâmetro $\alpha_{i}: \mathbb{R} \rightarrow \operatorname{Spin}_{n+1}$ é dado por $\alpha_{i}(\theta)=\cos \left(\frac{\theta}{2}\right)+\hat{a}_{i} \sin \left(\frac{\theta}{2}\right), \operatorname{com} i \in \llbracket n \rrbracket$.

Definição 4.4.1 O grupo gerado pelos elementos $\alpha_{i}(\theta)$, onde $\theta \in \mathbb{R}$ e $i \in \llbracket n \rrbracket$ é definido como $\operatorname{Spin}_{n+1}$.

Como definimos $\operatorname{Spin}_{n+1}$ a partir de seus geradores $\alpha_{i}(\theta)$, que são expressos matricialmente, então $\operatorname{Spin}_{n+1}$ pode ser visto como um grupo de matrizes.

Observação 4.4.1 Como $\alpha_{i}(\pi)=\hat{a}_{i}$, temos Quat $_{n+1} \subset \operatorname{Spin}_{n+1}$. Além disso, como $\mathrm{Cl}_{n+1}^{0}$ é gerada por $\mathrm{HQuat}_{n+1}$, temos Quat $_{n+1} \subset \operatorname{Spin}_{n+1} \subset \mathrm{Cl}_{n+1}^{0}$.

Uma vez que definimos $\alpha_{i}(\pi)=\hat{a}_{i}$, podemos definir em $\mathrm{Cl}_{n+1}^{0}$ os elementos

$$
\mathfrak{a}_{i}^{\text {Spin }}=\frac{1}{2} \hat{a}_{i}, i \in \llbracket n \rrbracket .
$$

Seja $\mathfrak{s p i n}_{\mathfrak{n}+1} \subset \mathrm{Cl}_{n+1}^{0}$ a álgebra de Lie gerada pelos elementos $\mathfrak{a}_{i}^{\text {Spin }}$. Existe um isomorfismo entre $\mathfrak{s p i n}_{n+1}$ e $\mathfrak{s o}_{n+1}$ como álgebras de Lie, portanto a dimensão de $\mathfrak{s p i n}_{n+1}$ é dada por $\frac{n(n+1)}{2}$, que é a dimensão de $\mathfrak{s o}_{n+1}$. Dessa forma, o grupo $\operatorname{Spin}_{n+1}$ tem a mesma dimensão.

Vamos ver alguns exemplos de grupos $\operatorname{Spin}_{n+1}$ conhecidos: $\operatorname{Spin}_{2}$ e $\operatorname{Spin}_{3}$.

Exemplo 4.4.1 $\operatorname{Spin}_{2}$ é gerado por $\left\{1, \alpha_{1}(\theta)\right\}$, portanto, se $z \in \operatorname{Spin}_{2} \subset \mathrm{Cl}_{2}^{0}$

$$
z=\cos \left(\frac{\theta}{2}\right)+\hat{a}_{1} \sin \left(\frac{\theta}{2}\right)
$$

entretanto, já vimos anteriormente que $\hat{a}_{1}=\mathbf{i}$ e $\mathrm{Cl}_{2}^{0}=\mathbb{C}$. Daí, como $\cos ^{2}\left(\frac{\theta}{2}\right)+\sin ^{2}\left(\frac{\theta}{2}\right)=1, z \in \operatorname{Spin}_{2}$ pode ser escrito como $z=x+\mathbf{i} y$ com $x^{2}+y^{2}=1$. Ou seja, é precisamente o grupo de matrizes ortogonais $\mathrm{SO}_{2}$. 
Anteriormente, vimos quem são os quatérnios $\mathbb{H}$, agora vamos pensar no grupo dos quatérnios unitários, que podem ser vistos como o grupo de matrizes reais $4 \times 4$ da forma

$$
M=\left(\begin{array}{cccc}
u & -v & -w & -t \\
v & u & -t & w \\
w & t & u & -v \\
t & -w & v & u
\end{array}\right),
$$

onde $\operatorname{det}(M)=\left(u^{2}+v^{2}+w^{2}+t^{2}\right)^{2}=1$.

Pensando nos elementos como 4-uplas $(u, v, w, t) \in \mathbb{R}^{4}$, eles formam uma forma análoga a esfera, já que $u^{2}+v^{2}+w^{2}+t^{2}=1$, chamada de 3-esfera $\mathbb{S}^{3} \subset \mathbb{R}^{4}$.

Exemplo 4.4.2 A álgebra de Clifford $\mathrm{Cl}_{3}^{0}$ é gerada por $\left\{1, \hat{a}_{1}, \hat{a}_{2}, \hat{a}_{1} \hat{a}_{2}\right\}$, tal que $\hat{a}_{1}^{2}=\hat{a}_{2}^{2}=-1$ e $\hat{a}_{1} \hat{a}_{2}=-\hat{a}_{2} \hat{a}_{1}$.

Sabemos que $\mathrm{Cl}_{3}^{0}=\mathbb{H}$, então como $\operatorname{Spin}_{3} \subset \mathrm{Cl}_{3}^{0}$, temos $\operatorname{Spin}_{3} \subset \mathbb{H}$. O grupo $\operatorname{Spin}_{3}$ é gerado por $\left\{ \pm 1, \pm \alpha_{1}(\theta), \pm \alpha_{2}(\theta)\right\}$, dados por

$$
\alpha_{1}(\theta)=\cos \left(\frac{\theta}{2}\right)+\hat{a}_{1} \sin \left(\frac{\theta}{2}\right), \quad \alpha_{2}(\theta)=\cos \left(\frac{\theta}{2}\right)+\hat{a}_{2} \sin \left(\frac{\theta}{2}\right) .
$$

Além disso,

$$
\alpha_{1}(\theta) \alpha_{2}(\theta)=\cos ^{2}\left(\frac{\theta}{2}\right)+\hat{a}_{1} \sin \left(\frac{\theta}{2}\right)+\hat{a}_{2} \sin \left(\frac{\theta}{2}\right) \cos \left(\frac{\theta}{2}\right)+\hat{a}_{1} \hat{a}_{2} \sin ^{2}\left(\frac{\theta}{2}\right),
$$

onde a soma dos quadrados dos coeficientes de $\alpha_{1}(\theta)$ e $\alpha_{2}(\theta)$ é $\cos ^{2}\left(\frac{\theta}{2}\right)+$ $\sin ^{2}\left(\frac{\theta}{2}\right)=1$, e a de $\alpha_{1}(\theta) \alpha_{2}(\theta)$ é $\left(\sin \left(\frac{\theta}{2}\right)^{2}+\cos \left(\frac{\theta}{2}\right)^{2}\right)^{2}=1$.

Podemos então concluir, que os elementos de $\mathrm{Spin}_{3}$ são escritos como $x+y \hat{a}_{1}+z \hat{a}_{2}+t \hat{a}_{1} \hat{a}_{2}$, onde $x^{2}+y^{2}+z^{2}+t^{2}=1$.

Daí, se $z \in \operatorname{Spin}_{3} \subset \mathbb{H}$, então $z=x+y \mathbf{i}+z \mathbf{j}+t \mathbf{k}$ onde $\mathbf{i}=\hat{a}_{1}, \mathbf{j}=\hat{a}_{2}$ e $\mathbf{k}=\hat{a}_{1} \hat{a}_{2}$, tal que $|z|=1$, ou seja, exatamente o grupo de matrizes dos quatérnios unitários $\mathbb{S}^{3}$. Assim, $\mathbb{S}^{3} \simeq \operatorname{Spin}_{3}$.

Observação 4.4.2 A multiplicação por um elemento do grupo $\operatorname{Spin}_{n+1}$, define uma transformação linear da álgebra de Clifford $\mathrm{Cl}_{n+1}^{0}$ nela mesma. A base HQuat $_{n+1}$ nos permite expressar esta transformação linear como uma matriz real $2^{n} \times 2^{n}$. 


\section{5}

\section{O Grupo $\tilde{\mathrm{B}}_{n+1}^{+}$}

Agora que temos bem definidos os grupos Quat $_{n+1}$ e $\operatorname{Spin}_{n+1}$, assim como seus geradores, podemos definir o grupo finito $\tilde{\mathrm{B}}_{n+1}^{+} \subset \operatorname{Spin}_{n+1}$.

Vamos então, definir os elementos $\dot{a}_{i}$ e $\grave{a}_{i}$, tal que $\left(\dot{a}_{i}\right)^{-1}=\grave{a}_{i}$ como

$$
\dot{a}_{i}=\alpha_{i}\left(\frac{\pi}{2}\right)=\frac{1+\hat{a}_{i}}{\sqrt{2}}, \quad \grave{a}_{i}=\alpha_{i}\left(\frac{-\pi}{2}\right)=\frac{1-\hat{a}_{i}}{\sqrt{2}},
$$

onde $\stackrel{a}{i}_{i}, \grave{a}_{i} \in \operatorname{Spin}_{n+1} \subset \mathrm{Cl}_{n+1}^{0}$.

Note que $\hat{a}_{i}=\hat{a}_{i}^{2}$ e $\hat{a}_{i}^{2}=a_{i}^{4}=-1$.

Definição 4.5.1 O grupo gerado pelos elementos $\left\{a_{1}, \ldots, a_{n}\right\}$ é definido como $\tilde{\mathrm{B}}_{n+1}^{+} \subset \operatorname{Spin}_{n+1}$.

Como tanto Quat ${ }_{n+1}$, quanto $\operatorname{Spin}_{n+1}$, são vistos como grupos de matrizes, naturalmente, $\tilde{\mathrm{B}}_{n+1}^{+}$também pode ser visto como tal.

Exemplo 4.5.1 Se $n=i=2$, então $\dot{a}_{2}, \grave{a}_{2} \in \tilde{\mathrm{B}}_{n+1}^{+}$são matrizes $4 \times 4$ dadas por

$$
\dot{a}_{2}=\left(\begin{array}{cccc}
\frac{\sqrt{2}}{2} & 0 & -\frac{\sqrt{2}}{2} & 0 \\
0 & \frac{\sqrt{2}}{2} & 0 & \frac{\sqrt{2}}{2} \\
\frac{\sqrt{2}}{2} & 0 & \frac{\sqrt{2}}{2} & 0 \\
0 & -\frac{\sqrt{2}}{2} & 0 & \frac{\sqrt{2}}{2}
\end{array}\right) \quad e \quad \grave{a}_{2}=\left(\begin{array}{cccc}
-\frac{\sqrt{2}}{2} & 0 & \frac{\sqrt{2}}{2} & 0 \\
0 & -\frac{\sqrt{2}}{2} & 0 & -\frac{\sqrt{2}}{2} \\
-\frac{\sqrt{2}}{2} & 0 & -\frac{\sqrt{2}}{2} & 0 \\
0 & \frac{\sqrt{2}}{2} & 0 & -\frac{\sqrt{2}}{2}
\end{array}\right)
$$

Note que as matrizes são ortogonais, com determinante 1. Além disso, os elementos de cada diagonal são iguais e se $i \neq j$ temos $a_{i j}=-a_{j i}$.

Considerando as definições acima para $\dot{a}_{i}$ e $\grave{a}_{i}$, podemos demonstrar o seguinte lema.

Lema 4.5.1 Para $\dot{a}_{i} \in \tilde{\mathrm{B}}_{n+1}^{+}$e $\hat{a}_{i} \in$ Quat $_{n+1}$, temos as seguintes identidades:

(i) Para todo $i \in \llbracket n-1 \rrbracket$ temos $\left\{\begin{array}{l}\dot{a}_{i} \hat{a}_{i+1} \dot{a}_{i}=\dot{a}_{i+1} \dot{a}_{i} \dot{a}_{i+1} \\ \left(\dot{a}_{i}\right)^{-1} \dot{a}_{i+1}\left(\dot{a}_{i}\right)^{-1}=\dot{a}_{i+1}\left(\dot{a}_{i}\right)^{-1} \dot{a}_{i+1}\end{array}\right.$;

(ii) $S e|i-j| \neq 1 \Longrightarrow\left\{\begin{array}{l}\dot{a}_{j} \hat{a}_{i}=\dot{a}_{i} \hat{a}_{j} \\ \hat{a}_{j} \hat{a}_{i}=\dot{a}_{i} \hat{a}_{j}\end{array}\right.$;

(iii) $S e|i-j|=1 \Longrightarrow\left\{\begin{array}{l}\hat{a}_{j} \hat{a}_{i}=\left(\hat{a}_{i}\right)^{-1} \hat{a}_{j} \\ \hat{a}_{j} \hat{a}_{i}=-\hat{a}_{i} \hat{a}_{j}\end{array}\right.$. 
Prova.

Utilizando as definições de $a_{i}$ e $\hat{a}_{i}$ dadas acima, basta fazermos algumas contas para verificar o resultado.

No primeiro item, vamos calcular os dois lados da primeira igualdade. À esquerda temos

$$
\begin{aligned}
\dot{a}_{i} \dot{a}_{i+1} \dot{a}_{i} & =\left(\frac{1+\hat{a}_{i}}{\sqrt{2}}\right)\left(\frac{1+\hat{a}_{i+1}}{\sqrt{2}}\right)\left(\frac{1+\hat{a}_{i}}{\sqrt{2}}\right) \\
& =\frac{1}{2 \sqrt{2}}\left(1+2 \hat{a}_{i}+\hat{a}_{i+1}+\hat{a}_{i+1} \hat{a}_{i}+\hat{a}_{i}^{2}+\hat{a}_{i} \hat{a}_{i+1}+\hat{a}_{i} \hat{a}_{i+1} \hat{a}_{i}\right),
\end{aligned}
$$

entretanto, como visto anteriormente, $\hat{a}_{i}^{2}=-1, \hat{a}_{i} \hat{a}_{i+1}=-\hat{a}_{i+1} \hat{a}_{i}$. Portanto,

$$
\dot{a}_{i} \dot{a}_{i+1} \dot{a}_{i}=\frac{1}{\sqrt{2}}\left(\hat{a}_{i}+\hat{a}_{i+1}\right)
$$

À direita temos

$$
\dot{a}_{i+1} \dot{a}_{i} \dot{a}_{i+1}=\frac{1}{2 \sqrt{2}}\left(1+\hat{a}_{i}+2 \hat{a}_{i+1}+\hat{a}_{i} \hat{a}_{i+1}+\hat{a}_{i+1}^{2}+\hat{a}_{i+1} \hat{a}_{i}+\hat{a}_{i+1} \hat{a}_{i} \hat{a}_{i+1}\right),
$$

dadas as relações anteriores, temos

$$
\dot{a}_{i+1} \dot{a}_{i} \dot{a}_{i+1}=\frac{1}{\sqrt{2}}\left(\hat{a}_{i}+\hat{a}_{i+1}\right) .
$$

Portanto, $\mathfrak{a}_{i} \dot{a}_{i+1} \dot{a}_{i}=\dot{a}_{i+1} \dot{a}_{i} \dot{a}_{i+1}$.

Para a segunda igualdade, basta fazer uma conta similar considerando $\left(\hat{a}_{i}\right)^{-1}=\grave{a}_{i}=\frac{1-\hat{a}_{i}}{\sqrt{2}}$.

Os itens (ii) e (iii) seguem de contas parecidas com as anteriores, porém ainda mais simples, basta observar que:

se $|i-j| \neq 1$, então $\hat{a}_{i} \hat{a}_{j}=\hat{a}_{j} \hat{a}_{i}$ e se $|i-j|=1$, então $\hat{a}_{i} \hat{a}_{j}=-\hat{a}_{j} \hat{a}_{i}$.

O lema que acabamos de demonstrar, nos diz como os elementos $\hat{a}_{i}, \dot{a}_{i}$ e $\grave{a}_{i}$ interagem entre si. Tal interação é de extrema importância para os próximos capítulos.

\section{6}

O Homomorsfismo $\Pi: \operatorname{Spin}_{n+1} \rightarrow \mathrm{SO}_{n+1}$

O grupo $\operatorname{Spin}_{n+1}$, que definimos anteriormente, é também conhecido como o recobrimento duplo de $\mathrm{SO}_{n+1}$. Não abordaremos espaços de recobrimento neste trabalho, entretanto, é um tema de grande estudo, para saber mais sobre veja por exemplo, [8]. 
Lembre que como álgebras de Lie, existe um único isomorfismo entre $\mathfrak{s p i n}_{n+1}$ e $\mathfrak{s o}_{n+1}$, portanto, $\mathfrak{s p i n}_{n+1}$ tem dimensão finita e igual a $\frac{n(n+1)}{2}$. Além disso, vamos enunciar alguns resultados que irão nos auxiliar.

Teorema 4.6.1 (Terceiro teorema de Lie) Toda álgebra de Lie real de dimensão finita é a álgebra de Lie de um único grupo de Lie simplesmente conexo.

A demonstração do terceiro teorema de Lie pode ser encontrada em [10].

Teorema 4.6.2 Seja G um grupo de Lie conexo e seja $p: \tilde{G} \rightarrow G$ o mapa de recobrimento universal de G. Então, $\tilde{G}$ tem uma estrutura natural de grupo de Lie simplesmente conexo tal que, $p: \tilde{G} \rightarrow G$ é um homomorfismo de grupos de Lie.

A demonstração do teorema pode ser encontrada em [4].

Teorema 4.6.3 Suponha $G$ e $H$ grupos de Lie com álgebras de Lie $\mathfrak{g}$ e $\mathfrak{h}$, respectivamente. Além disso, suponha $f: \mathfrak{g} \rightarrow \mathfrak{h}$ homomorfismo de álgebras de Lie. Suponha também que G seja conexo e simplesmente conexo. Então, existe um único homomorfismo de grupos de Lie $\psi: G \rightarrow H$ tal que $\psi_{*}=f$, onde $\psi_{*}$ é a diferencial de $\psi$ na identidade.

A demonstração do teorema pode ser encontrada em [7].

Pelos resultados acima, $\operatorname{Spin}_{n+1}$ é simplesmente conexo e existe um único homomorfismo $\Pi: \operatorname{Spin}_{n+1} \rightarrow \mathrm{SO}_{n+1}$, tal que $\alpha_{i}^{\text {Spin }}(\theta) \mapsto \alpha_{i}^{\mathrm{SO}}(\theta)$, ou seja,

$$
\begin{aligned}
\Pi: \operatorname{Spin}_{n+1} & \rightarrow \mathrm{SO}_{n+1} \\
\alpha_{i}(\theta) & \mapsto\left(\begin{array}{lll}
I_{1} & & \\
& \operatorname{Rot}(\theta) & \\
& & I_{2}
\end{array}\right),
\end{aligned}
$$

onde $I_{1} \in \mathbb{R}^{(i-1) \times(i-1)}$ e $I_{2} \in \mathbb{R}^{(n-i) \times(n-i)}$ são matrizes identidade. Além disso, $\operatorname{Rot}(\theta)$ é a matriz de rotação $2 \times 2$ dada por

$$
\operatorname{Rot}(\theta)=\left(\begin{array}{cc}
\cos (\theta) & -\sin (\theta) \\
\sin (\theta) & \cos (\theta)
\end{array}\right) .
$$

Antes de continuarmos, vamos ver um exemplo para entender melhor como funciona este mapa.

Exemplo 4.6.1 Se $n=3$, então $\mathrm{Spin}_{4}$ é um grupo de matrizes $8 \times 8$ e $\mathrm{SO}_{4}$ é um grupo de matrizes $4 \times 4$. Temos os geradores $\alpha_{i}(\theta)$, com $i \in\{1,2,3\}$. 
Seja $i=3$, dai $\Pi\left(\alpha_{3}\right)$ é uma matriz em $\mathrm{SO}_{4}$, cujo bloco Rot $(\theta)$ ocupa as entradas $(3,3),(3,4),(4,3)$ e $(4,4)$. Então

$$
\Pi\left(\alpha_{3}(\theta)\right)=\left(\begin{array}{cccc}
1 & 0 & 0 & 0 \\
0 & 1 & 0 & 0 \\
0 & 0 & \cos (\theta) & -\sin (\theta) \\
0 & 0 & \sin (\theta) & \cos (\theta)
\end{array}\right)
$$

Continuando no exemplo, vamos analisar o que acontece quando $\theta=\pi$, ou seja, $\alpha_{i}(\pi)=\hat{a}_{i}$ e quando $\theta=\frac{\pi}{2}$, ou seja, $\alpha_{i}\left(\frac{\pi}{2}\right)=\dot{a}_{i}$.

Para isto, basta aplicarmos os ângulos na matriz do exemplo. Dessa forma, temos

$$
\Pi\left(\hat{a}_{3}\right)=\left(\begin{array}{cccc}
1 & 0 & 0 & 0 \\
0 & 1 & 0 & 0 \\
0 & 0 & -1 & 0 \\
0 & 0 & 0 & -1
\end{array}\right) \quad \text { e } \Pi\left(\dot{a}_{3}\right)=\left(\begin{array}{cccc}
1 & 0 & 0 & 0 \\
0 & 1 & 0 & 0 \\
0 & 0 & 0 & -1 \\
0 & 0 & 1 & 0
\end{array}\right) .
$$

Observe que,

$$
\operatorname{Rot}(\pi)=\left(\begin{array}{cc}
-1 & 0 \\
0 & -1
\end{array}\right) \quad \text { e } \quad \operatorname{Rot}\left(\frac{\pi}{2}\right)=\left(\begin{array}{cc}
0 & -1 \\
1 & 0
\end{array}\right)
$$

que são matrizes de determinante 1 , claramente.

Portanto, é fácil ver que o caso geral é dado por

$$
\Pi\left(\hat{a}_{i}\right)=\left(\begin{array}{cccc}
I_{1} & & & \\
& -1 & & \\
& & -1 & \\
& & & I_{2}
\end{array}\right) \text { e } \Pi\left(\hat{a}_{i}\right)=\left(\begin{array}{llll}
I_{1} & & & \\
& & -1 & \\
& 1 & & \\
& & & I_{2}
\end{array}\right) \text {. }
$$

Note que $\Pi\left(\hat{a}_{i}\right)$ é uma matriz diagonal de determinante 1. Além disso, $\Pi\left(\hat{a}_{i}\right)$ é uma matriz de permutação, também de determinante 1 .

Lembre que Quat ${ }_{n+1} \subset \operatorname{Spin}_{n+1}$ é gerado pelos $\hat{a}_{i}$ e $\tilde{\mathrm{B}}_{n+1}^{+} \subset \operatorname{Spin}_{n+1}$ é gerado pelos $\dot{a}_{i}$. Portanto,

$$
\Pi\left[\mathrm{Quat}_{n+1}\right] \subset \operatorname{Diag}_{n+1}^{+}, \Pi\left[\tilde{\mathrm{B}}_{n+1}^{+}\right] \subset \mathrm{B}_{n+1}^{+}
$$

Como claramente as inclusões contrárias são válidas, temos

$$
\Pi\left[\text { Quat }_{n+1}\right]=\operatorname{Diag}_{n+1}^{+}, \Pi\left[\tilde{\mathrm{B}}_{n+1}^{+}\right]=\mathrm{B}_{n+1}^{+} .
$$


Na seção 2.2, vimos que o mapa $\phi: \mathrm{B}_{n+1}^{+} \rightarrow \mathrm{S}_{n+1}$ é um homomorfismo sobrejetor, cujo núcleo é $\operatorname{Diag}_{n+1}^{+}$. Portanto,

$$
\frac{\mathrm{B}_{n+1}^{+}}{\operatorname{Diag}_{n+1}^{+}} \approx \mathrm{S}_{n+1} \text {. }
$$

Além disso, $\phi \circ \Pi=\sigma$ onde

$$
\begin{aligned}
\sigma: \tilde{\mathrm{B}}_{n+1}^{+} & \rightarrow \mathrm{S}_{n+1} \\
z & \mapsto \sigma_{z}
\end{aligned}
$$

é também um homomorfismo, cujo núcleo é Quat ${ }_{n+1}$.

Exemplo 4.6.2 No exemplo anterior, a permutação $\sigma \in \mathrm{S}_{4}$ associada a matriz $\Pi\left(\dot{a}_{3}\right)$ é dada por $\phi \circ \Pi\left(\dot{a}_{3}\right)=\sigma=[1243]=a_{3}$.

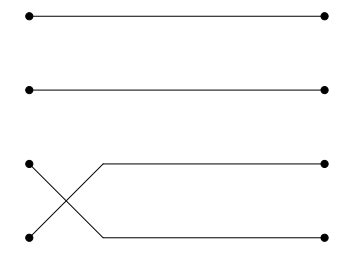

Figura 4.1: Diagrama de $\sigma=a_{3} \in \mathrm{S}_{4}$

Observação 4.6.1 Note que $\phi \circ \Pi\left(\dot{a}_{i}\right)=\sigma_{\dot{a}_{i}}=a_{i} \in \mathrm{S}_{n+1}$.

Feita a análise, temos que o mapa $\Pi: \operatorname{Spin}_{n+1} \rightarrow \mathrm{SO}_{n+1}$ nos fornece as seguintes sequências exatas, ou seja, homomorfismos encadeados em que a imagem do antecessor é o núcleo do sucessor:

(i) $1 \rightarrow$ Quat $_{n+1} \hookrightarrow \tilde{\mathrm{B}}_{n+1}^{+} \stackrel{\sigma}{\rightarrow} S_{n+1} \rightarrow 1$;

(ii) $1 \rightarrow\{ \pm 1\} \hookrightarrow$ Quat $_{n+1} \stackrel{\Pi}{\rightarrow} \operatorname{Diag}_{n+1}^{+} \rightarrow 1$,

onde $\tilde{\mathrm{B}}_{n+1}^{+}=\Pi^{-1}\left[\mathrm{~B}_{n+1}^{+}\right]$e Quat ${ }_{n+1}=\Pi^{-1}\left[\operatorname{Diag}_{n+1}^{+}\right]$.

Exemplo 4.6.3 Seja $z=a_{1} \dot{a}_{3} \dot{a}_{2} \in \tilde{\mathrm{B}}_{4}^{+}$, então

$$
\begin{gathered}
\Pi(z)=\Pi\left(\dot{a}_{1} \dot{a}_{3} \dot{a}_{2}\right)=\Pi\left(\dot{a}_{1}\right) \Pi\left(\dot{a}_{3}\right) \Pi\left(\dot{a}_{2}\right) \\
\Pi(z)=\left(\begin{array}{cccc}
0 & -1 & 0 & 0 \\
1 & 0 & 0 & 0 \\
0 & 0 & 1 & 0 \\
0 & 0 & 0 & 1
\end{array}\right) \cdot\left(\begin{array}{cccc}
1 & 0 & 0 & 0 \\
0 & 1 & 0 & 0 \\
0 & 0 & 0 & -1 \\
0 & 0 & 1 & 0
\end{array}\right) \cdot\left(\begin{array}{cccc}
1 & 0 & 0 & 0 \\
0 & 0 & -1 & 0 \\
0 & 1 & 0 & 0 \\
0 & 0 & 0 & 1
\end{array}\right)=\left(\begin{array}{cccc}
0 & 0 & 1 & 0 \\
1 & 0 & 0 & 0 \\
0 & 0 & 0 & -1 \\
0 & 1 & 0 & 0
\end{array}\right),
\end{gathered}
$$

$\operatorname{com} \phi(\Pi(z))=[3142]=a_{1} a_{3} a_{2}$. 
Como nosso objetivo é estudar permutações $\sigma \in \mathrm{S}_{n+1}$ com as coordenadas de $z \in \tilde{\mathrm{B}}_{n+1}^{+}$, onde $\phi \circ \Pi(z)=\sigma$, é importante que tenhamos compreendido o funcionamento do mapa $\Pi$ nos geradores $\dot{a}_{i} \in \tilde{\mathrm{B}}_{n+1}^{+}$de forma clara.

Com o homomorfismo bem entendido, a partir de então, omitiremos $\phi$ e trataremos apenas como $\sigma=\Pi(z)$. 


\section{Permutações e a Álgebra de Clifford}

No capítulo anterior, vimos os grupos $\operatorname{Spin}_{n+1}$, Quat ${ }_{n+1}$ e $\tilde{\mathrm{B}}_{n+1}^{+}$. No presente capítulo, estudaremos um pouco mais a álgebra $\mathrm{Cl}_{n+1}^{0}$ e suas propriedades, a partir de seus geradores. Em seguida, estudaremos as permutações em $\mathrm{S}_{n+1}$ nas coordenadas de $z \in \tilde{\mathrm{B}}_{n+1}^{+} \subset \mathrm{Cl}_{n+1}^{0}$.

\section{1}

\section{Os Mapas Acute e Grave}

Vimos no capítulo 2, que podemos escrever $\sigma \in \mathrm{S}_{n+1}$ como uma palavra reduzida, que é um produto de geradores $a_{i}$ de $\mathrm{S}_{n+1}$.

Definiremos os mapas acute e grave por meio das palavras reduzidas, utilizando os elementos que já conhecemos $\dot{a}_{i}, \grave{a}_{i} \in \tilde{\mathrm{B}}_{n+1}^{+} \subset \mathrm{Cl}_{n+1}^{0}$.

Definição 5.1.1 Seja $\sigma \in S_{n+1}$, tal que $\sigma=a_{i_{1}} \ldots a_{i_{l}}$ é uma palavra reduzida. Seja $\grave{a}_{i}=\left(\grave{a}_{i}\right)^{-1}$. Definimos os seguintes mapas :

(i) acute $: S_{n+1} \rightarrow \tilde{\mathrm{B}}_{n+1}^{+}$, dado por acute $(\sigma)=\dot{\sigma}=\dot{a}_{i_{1}} \ldots \dot{a}_{i_{l}}$;

(ii) grave $: S_{n+1} \rightarrow \tilde{\mathrm{B}}_{n+1}^{+}$, dado por grave $(\sigma)=\grave{\sigma}=\grave{a}_{i_{1}} \ldots \grave{a}_{i_{l}}$.

A princípio, a definição parece depender da palavra reduzida escolhida. O lema abaixo nos mostra que os mapas são bem definidos, portanto não existe tal dependência.

Lema 5.1.1 Se $\sigma \in S_{n+1}$ é tal que $\sigma=a_{i_{1}} \ldots a_{i_{l}}=a_{j_{1}} \ldots a_{j_{l}}$ e $l=\operatorname{inv}(\sigma)$, então $\dot{a}_{i_{1}} \ldots \dot{a}_{i_{l}}=\dot{a}_{j_{1}} \ldots \dot{a}_{j_{l}}$.

Prova.

Pela proposição da seção 2.1, duas palavras reduzidas estão conectadas por uma sequência finita de movimentos. Além disso, pelo lema da seção 4.5, para cada movimento da sequência, temos as identidades.

Portanto, $\dot{a}_{i_{1}} \ldots \dot{a}_{i_{l}}=\dot{a}_{j_{1}} \ldots \dot{a}_{j_{l}}$.

Recordemos das relações já estabelecidas,

$$
\alpha_{i}(\theta)=\cos \left(\frac{\theta}{2}\right)+\hat{a}_{i} \sin \left(\frac{\theta}{2}\right) \in \operatorname{Spin}_{n+1}, \quad \alpha_{i}(\pi)=\hat{a}_{i} \in \text { Quat }_{n+1},
$$




$$
\alpha_{i}\left(\frac{\pi}{2}\right)=\frac{1+\hat{a}_{i}}{\sqrt{2}}=\dot{a}_{i} \in \tilde{\mathrm{B}}_{n+1}^{+}, \quad \alpha_{i}\left(\frac{-\pi}{2}\right)=\frac{1-\hat{a}_{i}}{\sqrt{2}}=\grave{a}_{i} \in \tilde{\mathrm{B}}_{n+1}^{+} .
$$

Além disso, $\left(\hat{a}_{i}\right)^{4}=\left(\hat{a}_{i}\right)^{2}=-1$.

Exemplo 5.1.1 Seja $\sigma=\eta=[654321]=a_{1} a_{2} a_{1} a_{3} a_{2} a_{1} a_{4} a_{3} a_{2} a_{1} a_{5} a_{4} a_{3} a_{2} a_{1}$.

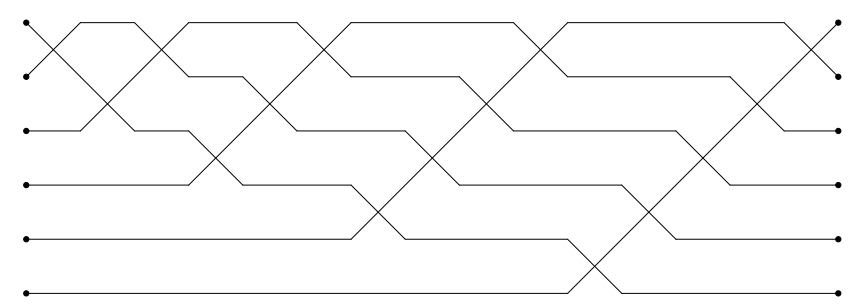

Figura 5.1: Diagrama de $\eta \in \mathrm{S}_{6}$

Lembre que $\dot{a}_{i}=\frac{1+\hat{a}_{i}}{\sqrt{2}}$, portanto temos

$$
\begin{aligned}
\dot{\eta} & =\dot{a}_{1} \hat{a}_{2} \dot{a}_{1} \dot{a}_{3} \dot{a}_{2} \dot{a}_{1} \dot{a}_{4} \hat{a}_{3} \dot{a}_{2} \dot{a}_{1} \dot{a}_{5} \dot{a}_{4} \hat{a}_{3} \dot{a}_{2} \dot{a}_{1} \\
& =\left(\frac{1+\hat{a}_{1}}{\sqrt{2}}\right) \ldots\left(\frac{1+\hat{a}_{1}}{\sqrt{2}}\right) .
\end{aligned}
$$

Assim, tendo em mente as relações entre $\hat{a}_{i}, \grave{a}_{i}$ e $\grave{a}_{i}$, após alguns cálculos concluímos que

$$
\dot{\eta}=\frac{1}{2 \sqrt{2}}\left(1+\hat{a}_{3}-\hat{a}_{2} \hat{a}_{4}-\hat{a}_{2} \hat{a}_{3} \hat{a}_{4}-\hat{a}_{1} \hat{a}_{5}-\hat{a}_{1} \hat{a}_{3} \hat{a}_{5}+\hat{a}_{1} \hat{a}_{2} \hat{a}_{4} \hat{a}_{5}+\hat{a}_{1} \hat{a}_{2} \hat{a}_{3} \hat{a}_{4} \hat{a}_{5}\right) .
$$

\section{2}

\section{Parte Real}

Vimos que Quat ${ }_{n+1} \subset \operatorname{Spin}_{n+1} \subset \mathrm{Cl}_{n+1}^{0}$. Além disso, um elemento de $\mathrm{Cl}_{n+1}^{0}$ é escrito como uma combinação linear de elementos de Quat $_{n+1}$.

Portanto, podemos escrever $z \in \mathrm{Cl}_{n+1}^{0}$ como

$$
z=\sum_{q \in \mathrm{HQuat}_{n+1}} c_{q} q, \quad \operatorname{com} c_{q} \in \mathbb{R}
$$

Definição 5.2.1 A parte real de $z \in \mathrm{Cl}_{n+1}^{0}$ é definida como

$$
\mathfrak{R}(z)=2^{-n} \operatorname{Traço}(z)=\langle z, 1\rangle
$$

Portanto, para $z=\sum_{q \in \mathrm{HQuat}_{n+1}} c_{q} q \in \mathrm{Cl}_{n+1}^{0}$ a parte real é o coeficiente independente $\mathfrak{R}(z)=c_{1}$.

Exemplo 5.2.1 No exemplo 5.1.1 da seção anterior, onde $\dot{\eta} \in \mathrm{Cl}_{6}^{0}$ é tal que

$$
\dot{\eta}=\frac{1}{2 \sqrt{2}}\left(1+\hat{a}_{3}-\hat{a}_{2} \hat{a}_{4}-\hat{a}_{2} \hat{a}_{3} \hat{a}_{4}-\hat{a}_{1} \hat{a}_{5}-\hat{a}_{1} \hat{a}_{3} \hat{a}_{5}+\hat{a}_{1} \hat{a}_{2} \hat{a}_{4} \hat{a}_{5}+\hat{a}_{1} \hat{a}_{2} \hat{a}_{3} \hat{a}_{4} \hat{a}_{5}\right),
$$


temos $\mathfrak{R}(\dot{\eta})=\frac{1}{2 \sqrt{2}}$.

Exemplo 5.2.2 Seja $\sigma=[2413]=a_{2} a_{1} a_{3} \in \mathrm{S}_{4}$, então

$$
\dot{\sigma}=\frac{1}{2 \sqrt{2}}\left(1+\hat{a}_{1}+\hat{a}_{2}+\hat{a}_{3}+\hat{a}_{1} \hat{a}_{3}+\hat{a}_{2} \hat{a}_{3}+\hat{a}_{2} \hat{a}_{1}+\hat{a}_{2} \hat{a}_{1} \hat{a}_{3}\right)
$$

Portanto, $\mathfrak{R}(\dot{\sigma})=\frac{1}{2 \sqrt{2}}$.

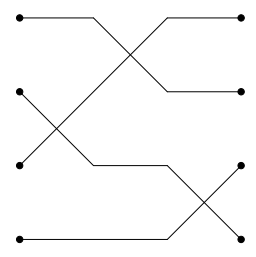

Figura 5.2: Diagrama de $\sigma=a_{2} a_{1} a_{3} \in \mathrm{S}_{4}$

Exemplo 5.2.3 Seja $z=-\hat{a}_{1} \dot{\sigma} \in \mathrm{Spin}_{4}$, onde $\sigma=a_{2} a_{1} a_{3} \in \mathrm{S}_{4}$ como no exemplo anterior. Então

$$
z=\frac{1}{4}\left(1-\hat{a}_{1}+\hat{a}_{2}+\hat{a}_{3}-\hat{a}_{1} \hat{a}_{2}-\hat{a}_{1} \hat{a}_{3}-\hat{a}_{1} \hat{a}_{2} \hat{a}_{3}-\hat{a}_{1} \hat{a}_{2} \hat{a}_{1} \hat{a}_{3}\right),
$$

portanto $\mathfrak{R}(z)=\frac{1}{4}$.

\subsection{1}

\section{Autovalores e a Parte Real}

Recordemos do homomorfismo $\Pi: \operatorname{Spin}_{n+1} \rightarrow \mathrm{SO}_{n+1}$, da seção 4.6, onde vimos que $\Pi\left[\tilde{\mathrm{B}}_{n+1}^{+}\right]=\mathrm{B}_{n+1}^{+}$e $\Pi\left[\right.$ Quat $\left._{n+1}\right]=\operatorname{Diag}_{n+1}^{+}$.

Veremos um resultado que relaciona a parte real de $z \in \operatorname{Spin}_{n+1} \subset \mathrm{Cl}_{n+1}^{0}$, com os autovalores da matriz $\Pi(z) \in \mathrm{SO}_{n+1}$.

Lema 5.2.1 Para $z \in \operatorname{Spin}_{n+1} \subset \mathrm{Cl}_{n+1}^{0}$, seja $Q=\Pi(z) \in \mathrm{SO}_{n+1}$ tal que seus autovalores são $\exp \left( \pm \theta_{1} i\right), \ldots, \exp \left( \pm \theta_{k} i\right), 1, \ldots, 1$. Então

$$
\mathfrak{R}(z)= \pm \cos \left(\frac{\theta_{1}}{2}\right) \ldots \cos \left(\frac{\theta_{k}}{2}\right)
$$

Em particular, $\mathfrak{R}(z)=0$ se, e somente se, -1 é um autovalor de $Q$.

Prova.

Sejam $z \in \operatorname{Spin}_{n+1}$ e $Q=\Pi(z) \in \mathrm{SO}_{n+1}$, tal que os seus autovalores são $\exp \left( \pm \theta_{1} i\right), \ldots, \exp \left( \pm \theta_{k} i\right), 1, \ldots, 1$. Daí, $Q$ é diagonalizável, ou seja, existe $P \in \mathrm{GL}_{n+1}$ tal que $Q=P D P^{-1}$, onde $D$ é a matriz diagonal dos autovalores de $Q$. 
Temos que $\mathfrak{R}: \operatorname{Spin}_{n+1} \rightarrow \mathbb{R}$ é invariante por conjugação, portanto se $z=z_{0} z_{1} z_{0}^{-1}$, temos $\mathfrak{R}(z)=\mathfrak{R}\left(z_{1}\right)$, onde $D=\Pi\left(z_{1}\right), P=\Pi\left(z_{0}\right)$ e $P^{-1}=\Pi\left(z_{0}^{-1}\right)$. Assim, podemos assumir $z=\alpha_{1}\left(\theta_{1}\right) \ldots \alpha_{2 k-1}\left(\theta_{k}\right)$ e $Q$ como a diagonal dos autovalores

$$
Q=\left(\begin{array}{ccccccc}
\cos \left(\theta_{1}\right) & -\sin \left(\theta_{1}\right) & & & & & \\
\sin \left(\theta_{1}\right) & \cos \left(\theta_{1}\right) & & & & \\
& & \ddots & & & \\
& & & \cos \left(\theta_{k}\right) & -\sin \left(\theta_{k}\right) & & \\
& & \sin \left(\theta_{k}\right) & \cos \left(\theta_{k}\right) & & \\
& & & & 1 & \\
& & & & \ddots & \\
& & & & & 1
\end{array}\right)
$$

Como $\alpha_{i}(\theta)=\cos \left(\frac{\theta}{2}\right)+\hat{a}_{i} \sin \left(\frac{\theta}{2}\right)$, então o coeficiente independente do produto dos $\alpha_{i}$ é o produto dos cossenos, portanto

$$
\mathfrak{R}\left(\alpha_{1}\left(\theta_{1}\right) \ldots \alpha_{2 k-1}\left(\theta_{k}\right)\right)= \pm \cos \left(\frac{\theta_{1}}{2}\right) \ldots \cos \left(\frac{\theta_{k}}{2}\right) .
$$

Note que se $\theta_{i}= \pm \pi$ para algum $i$, temos $\cos \left( \pm \frac{\pi}{2}\right)=0$, portanto $\mathfrak{R}(z)=0$. Reciprocamente, se $\mathfrak{R}(z)=0$ existe um $\theta_{i}$ tal que $\cos \left(\theta_{i}\right)=0$, daí $\theta_{i}= \pm \pi$. Logo, se $\theta_{i}= \pm \pi$ para algum $i$, temos $\exp ( \pm \pi i)=-1$.

Portanto, $\mathfrak{R}(z)=0$ se, e somente se, -1 é um autovalor de $Q=\Pi(z)$.

Exemplo 5.2.4 Seja $z \in \operatorname{Spin}_{4}$, tal que $\left\{\exp \left( \pm \frac{\pi}{2} i\right), \exp \left( \pm \frac{\pi}{3} i\right)\right\}$ é o conjunto de autovalores de $Q=\Pi(z) \in \mathrm{SO}_{4}$.

Dessa forma, podemos assumir

$$
Q=\left(\begin{array}{cccc}
\cos \left(\frac{\pi}{2}\right) & -\sin \left(\frac{\pi}{2}\right) & & \\
\sin \left(\frac{\pi}{2}\right) & \cos \left(\frac{\pi}{2}\right) & & \\
& & \cos \left(\frac{\pi}{3}\right) & -\sin \left(\frac{\pi}{3}\right) \\
& & \sin \left(\frac{\pi}{3}\right) & \cos \left(\frac{\pi}{3}\right)
\end{array}\right)=\left(\begin{array}{ccc} 
& & \\
1 & & \\
& \frac{1}{2} & -\frac{\sqrt{3}}{2} \\
& \frac{\sqrt{3}}{2} & \frac{1}{2}
\end{array}\right)
$$

e $z=\alpha_{1}\left(\frac{\pi}{2}\right) \alpha_{3}\left(\frac{\pi}{3}\right)$. Portanto, pelo lema anterior,

$$
\mathfrak{R}(z)=\cos \left(\frac{\pi}{4}\right) \cos \left(\frac{\pi}{6}\right)=\frac{\sqrt{2}}{2} \frac{\sqrt{3}}{2}=\frac{\sqrt{6}}{4} .
$$




\section{2 .2}

\section{Matrizes de Ciclos e a Parte Real}

Vimos pelo lema anterior, que podemos calcular $\mathfrak{R}(z)$ a partir de informações sobre $Q=\Pi(z) \in \mathrm{SO}_{n+1}$. Agora restringiremos um pouco, para ver como calcular $\mathfrak{R}(z)$ a partir de informações sobre $Q \in \mathrm{B}_{n+1}^{+}$.

Antes, vamos definir os polinômios de Chebyshev (veja [9]), que são ferramentas úteis na demonstração do próximo corolário.

Definição 5.2.2 Os polinômios de Chebyshev $T_{k}$ são definidos recursivamente por

$$
T_{0}(x)=1, \quad T_{1}(x)=x, \quad T_{k+1}(x)=2 x T_{k}(x)-T_{k-1}(x)
$$

e satisfazem $T_{k}(\cos (t))=\cos (k t)$. Portanto, para $k \geq 0$, o termo líder de $T_{k}(x)$ é $2^{k-1} x^{k}$ e o termo independente é 0 se $k$ é ímpar $e(-1)^{\frac{k}{2}}$ se $k$ é par.

As raízes de $T_{k}(x)$ são dadas por

$$
x_{l}=\cos \left(\frac{\pi(2 l-1)}{2 k}\right), \operatorname{com} l=1, \ldots, k .
$$

Além disso, $T_{k}(x)$ atinge seu máximo em

$$
y_{l}=\cos \left(\frac{l \pi}{k}\right), \operatorname{com} T_{k}\left(y_{l}\right)=(-1)^{l} \text { para cada } l=0, \ldots, k .
$$

Definição 5.2.3 Dizemos que uma matriz $Q \in \mathrm{B}_{n+1}^{+}$é um ciclo de comprimento $k$ par, quando existem $i_{1}, \ldots, i_{k}$ com

(i) $\left(e_{i_{k}}\right)^{T} Q=-\left(e_{i_{1}}\right)^{T}$,

(ii) $\left(e_{i_{j}}\right)^{T} Q=\left(e_{i_{j+1}}\right)^{T}$ para $1 \leq j<k$,

(iii) $\left(e_{j}\right)^{T} Q=\left(e_{j}\right)^{T}$ para $j>k$.

Se o comprimento for impar, $\left(e_{i_{k}}\right)^{T} Q=\left(e_{i_{1}}\right)^{T}$ e as relações (ii) e (iii) se mantém.

Exemplo 5.2.5 A matriz abaixo é uma matriz de ciclo de comprimento 3

$$
Q=\left(\begin{array}{lllll}
0 & 0 & 1 & 0 & 0 \\
0 & 1 & 0 & 0 & 0 \\
0 & 0 & 0 & 0 & 1 \\
0 & 0 & 0 & 1 & 0 \\
1 & 0 & 0 & 0 & 0
\end{array}\right)
$$

Na notação da definição temos, $i_{1}=1, i_{2}=3$ e $i_{3}=5$. Note que as condições da definição são verificadas facilmente. 
Corolário 5.2.1 Seja $z_{0} \in \tilde{\mathrm{B}}_{n+1}^{+}$, tal que $\Pi\left(z_{0}\right)=Q_{0} \in \mathrm{B}_{n+1}^{+}$seja um ciclo de comprimento k. Então $\mathfrak{R}\left(z_{0}\right)= \pm 2^{\frac{-k+1}{2}}$.

Prova.

Como $Q_{0} \in \mathrm{SO}_{n+1}$, então se $\lambda$ é autovalor de $Q_{0}$, temos $|\lambda|=1$.

Vamos separar em dois casos. Primeiramente, seja $k$ ímpar.

Como $Q_{0}$ é um ciclo de comprimento $\mathrm{k}$, temos $Q_{0}^{k}=I$, daí $\lambda^{k}=1$ onde k é o comprimento do ciclo. Então o polinômio característico de $Q_{0}$ é $p(\lambda)=\left(\lambda^{k}-1\right)(\lambda-1)^{n+1-k}$. Os autovalores simples dessa matriz são portanto, as raízes da unidade $\lambda=\exp \left(\frac{2 \pi i n}{k}\right)$, para $n=0,1, \ldots, k-1$.

Dessa forma, os autovalores de $Q_{0}$ são 1 , com a devida multiplicadade, e os autovalores simples

$$
\exp \left( \pm \frac{2 \pi i}{k}\right), \exp \left( \pm \frac{4 \pi i}{k}\right), \ldots, \exp \left( \pm \frac{(k-3) \pi i}{k}\right), \exp \left( \pm \frac{(k-1) \pi i}{k}\right)
$$

Pelo lema anterior, temos que

$$
\mathfrak{R}\left(z_{0}\right)= \pm \cos \left(\frac{2 \pi}{2 k}\right) \cos \left(\frac{4 \pi}{2 k}\right) \ldots \cos \left(\frac{(k-3) \pi}{2 k}\right) \cos \left(\frac{(k-1) \pi}{2 k}\right)= \pm P, \quad P>0 .
$$

Vimos que $T_{2 k}(x)-1=2^{2 k-1} x^{2 k}+\cdots-2$, então as raízes de $T_{2 k}(x)-1=0$ são $x_{l}=\cos \left(\frac{l \pi}{k}\right)$, com $l=0,1, \ldots, 2 k-1$, daí $-P^{4}$ é o produto das raízes de $T_{2 k}(x)-1=0$.

Portanto, como para $T_{2 k}(x)-1=0$ o termo independente é -2 e o coeficiente líder é $2^{2 k-1}$, então o produto das raízes de $T_{2 k}(x)-1$ é $\frac{(-2)}{2^{2 k-1}}=$ $-2^{-2 k+2}$. Logo, $P=2^{\frac{-k+1}{2}}$, portanto $\mathfrak{R}\left(z_{0}\right)= \pm 2^{\frac{-k+1}{2}}$.

Seja agora, $k$ par.

Como $Q_{0}$ é um ciclo de comprimento k temos $Q_{0}^{k}=J$, onde $J$ é uma matriz que difere da identidade em apenas uma entrada cujo valor é 1. Assim, como $k$ é par, $\lambda^{k}=-1$. Então o polinômio característico de $Q_{0}$ é $p(\lambda)=\left(\lambda^{k}+1\right)(\lambda-1)^{n+1-k}$. Os autovalores dessa matriz são portanto, $\lambda=\exp \left(\frac{(2 n-1) \pi i}{k}\right)$, para $n=1, \ldots, k$.

Dessa forma, os autovalores de $Q_{0}$ são 1 , com a devida multiplicadade, e os autovalores simples

$$
\exp \left( \pm \frac{\pi i}{k}\right), \exp \left( \pm \frac{3 \pi i}{k}\right), \ldots, \exp \left( \pm \frac{(k-1) \pi i}{k}\right) .
$$

Pelo lema anterior, temos que

$$
\mathfrak{R}\left(z_{0}\right)= \pm \cos \left(\frac{\pi}{2 k}\right) \cos \left(\frac{3 \pi}{2 k}\right) \ldots \cos \left(\frac{(k-1) \pi}{2 k}\right)= \pm P
$$


Como as raízes de $T_{k}(x)$ são dadas por $x_{l}=\cos \left(\frac{\pi(2 l-1)}{2 k}\right), \operatorname{com} l=1, \ldots, k$, daí $P^{2}$ é o produto das raízes de $T_{k}(x)$. O termo independente de $T_{k}(x)$ é $(-1)^{\frac{k}{2}}$ e o coeficiente líder é $2^{k-1}$, então o produto das raízes de $T_{k}(x)$ é $\frac{(-1)^{\frac{k}{2}}}{2^{k-1}}=2^{-k+1}$.

Portanto, $P^{2}=2^{-k+1}$ e assim $\mathfrak{R}\left(z_{0}\right)= \pm 2^{\frac{-k+1}{2}}$.

Exemplo 5.2.6 Seja $z=\dot{a}_{3} \dot{a}_{2} \in \tilde{\mathrm{B}}_{4}^{+}$. Daí, $\sigma=\phi \circ \Pi(z)=a_{3} a_{2}=(234) \in \mathrm{S}_{4}$, tal que $\Pi(z)=Q \in \mathrm{B}_{4}^{+}$é a matriz de permutação de $\sigma$, dada por

$$
Q=\left(\begin{array}{llll}
1 & 0 & 0 & 0 \\
0 & 0 & 1 & 0 \\
0 & 0 & 0 & 1 \\
0 & 1 & 0 & 0
\end{array}\right)
$$

Como $\sigma$ é um ciclo de comprimento 3, os autovalores de $Q$ são: $\exp \left( \pm \frac{2 \pi i}{3}\right)$ e 1. Portanto, pelo lema anterior

$$
\mathfrak{R}(z)= \pm \cos \left(\frac{\pi}{3}\right)= \pm\left(\frac{1}{2}\right)= \pm 2^{\frac{-k+1}{2}}, \quad \text { com } k=3 .
$$

Para compararmos os resultados, note que fazendo a conta braçal obtemos

$$
z=\dot{a}_{3} \dot{a}_{2}=\left(\frac{1+\hat{a}_{3}}{\sqrt{2}}\right)\left(\frac{1+\hat{a}_{2}}{\sqrt{2}}\right)=\frac{1}{2}\left(1+\hat{a}_{2}+\hat{a}_{3}+\hat{a}_{3} \hat{a}_{2}\right) .
$$

Portanto, $\mathfrak{R}(z)=\frac{1}{2}$.

Agora, vamos ver um exemplo onde o ciclo tem comprimento par.

Exemplo 5.2.7 Seja $z=\dot{a}_{3} \hat{a}_{2} \dot{a}_{1} \in \tilde{\mathrm{B}}_{4}^{+}$. Daí, $\sigma=a_{3} a_{2} a_{1}=(1234) \in \mathrm{S}_{4}$, tal que $\Pi(z)=Q \in \mathrm{B}_{4}^{+}$é a matriz de permutação de $\sigma$, dada por

$$
Q=\left(\begin{array}{cccc}
0 & 1 & 0 & 0 \\
0 & 0 & 1 & 0 \\
0 & 0 & 0 & 1 \\
-1 & 0 & 0 & 0
\end{array}\right)
$$

Como $\sigma$ é um ciclo de comprimento 4, então os autovalores de $Q$ são: $\exp \left( \pm \frac{\pi i}{4}\right), \exp \left( \pm \frac{3 \pi i}{4}\right), 1$.

Pelo lema anterior, recordando que $\cos \left(\frac{\theta}{2}\right)=\sqrt{\frac{1+\cos (\theta)}{2}}$, temos

$$
\mathfrak{R}(z)=\cos \left(\frac{\pi}{8}\right) \cos \left(\frac{3 \pi}{8}\right)= \pm \sqrt{\frac{1+\cos \left(\frac{\pi}{4}\right)}{2}} \sqrt{\frac{1+\cos \left(\frac{3 \pi}{4}\right)}{2}}=\frac{1}{2 \sqrt{2}}
$$


Para compararmos os resultados, vamos fazer a conta assim como no exemplo anterior. Temos,

$$
\begin{aligned}
z & =\dot{a}_{3} \dot{a}_{2} \dot{a}_{1}=\left(\frac{1+\hat{a}_{3}}{\sqrt{2}}\right)\left(\frac{1+\hat{a}_{2}}{\sqrt{2}}\right)\left(\frac{1+\hat{a}_{1}}{\sqrt{2}}\right) \\
& =\frac{1}{2 \sqrt{2}}\left(1+\hat{a}_{1}+\hat{a}_{2}+\hat{a}_{3}+\hat{a}_{3} \hat{a}_{2}+\hat{a}_{2} \hat{a}_{1}+\hat{a}_{3} \hat{a}_{1}+\hat{a}_{3} \hat{a}_{2} \hat{a}_{1}\right)
\end{aligned}
$$

Portanto, $\mathfrak{R}(z)=\frac{1}{2 \sqrt{2}}$.

\section{2 .3}

\section{Ciclos e a Parte Real}

Uma outra forma para calcular a parte real, nesse caso de um elemento de $\operatorname{Spin}_{n+1}$ de um tipo específico, é utilizando o número de ciclos de uma permutação em $\mathrm{S}_{n+1}$.

Antes de entendermos melhor o que queremos dizer, vamos ver alguns conceitos importantes para o resultado.

Temos as seguintes sequências exatas, do capítulo anterior:

$$
\begin{gathered}
1 \rightarrow \text { Quat }_{n+1} \hookrightarrow \tilde{\mathrm{B}}_{n+1}^{+} \stackrel{\sigma}{\rightarrow} S_{n+1} \rightarrow 1 ; \\
1 \rightarrow\{ \pm 1\} \hookrightarrow \text { Quat }_{n+1} \stackrel{\Pi}{\rightarrow} \operatorname{Diag}_{n+1}^{+} \rightarrow 1,
\end{gathered}
$$

onde $\Pi: \operatorname{Spin}_{n+1} \rightarrow \mathrm{SO}_{n+1}$ e $\phi \circ \Pi(z)=\sigma, \operatorname{com} \phi: \mathrm{B}_{n+1}^{+} \rightarrow \mathrm{S}_{n+1}$.

Temos que $\Pi^{-1}[\{\sigma\}]=$ ó quat $_{n+1} \subset \tilde{\mathrm{B}}_{n+1}^{+}$, ou seja, $\Pi\left[\hat{\sigma}\right.$ Quat $\left._{n+1}\right] \subset$ $\Pi\left[\tilde{\mathrm{B}}_{n+1}^{+}\right]=\mathrm{B}_{n+1}^{+}$. Da primeira sequência exata acima vemos que, para toda $\sigma \in \mathrm{S}_{n+1}$, o conjunto ó Quat ${ }_{n+1}$ é uma classe lateral.

Exemplo 5.2.8 Sejam $A, B \in \Pi\left[\tilde{\mathrm{B}}_{4}^{+}\right] \subset \Pi\left[\sigma\right.$ Quat $\left._{4}\right]$ matrizes dadas por

$$
A=\left(\begin{array}{cccc}
0 & 0 & 1 & 0 \\
1 & 0 & 0 & 0 \\
0 & 0 & 0 & -1 \\
0 & 1 & 0 & 0
\end{array}\right), \quad B=\left(\begin{array}{cccc}
0 & 0 & 1 & 0 \\
-1 & 0 & 0 & 0 \\
0 & 0 & 0 & -1 \\
0 & -1 & 0 & 0
\end{array}\right)
$$

Então, $\sigma=\phi(A)=\phi(B)=a_{1} a_{3} a_{2}$.

Do exemplo 4.6.3, sabemos que para $z=\dot{\sigma}=a_{1} a_{3} a_{2} \in \tilde{\mathrm{B}}_{4}^{+}$temos que $\Pi(z)=$ A. Para descobrirmos qual elemento $z_{1} \in \tilde{\mathrm{B}}_{4}^{+}$é tal que $\Pi\left(z_{1}\right)=B$, basta descobrirmos qual é a mudança de sinais necessária. Ou seja, queremos encontrar a matriz $C \in \operatorname{Diag}_{4}^{+}$tal que $A C=B$. 
Basta então, observarmos quais linhas mudam de sinal, no nosso caso as colunas 1 e 2. Dessa forma

$$
C=\left(\begin{array}{cccc}
-1 & 0 & 0 & 0 \\
0 & -1 & 0 & 0 \\
0 & 0 & 1 & 0 \\
0 & 0 & 0 & 1
\end{array}\right)
$$

Note que $C=\Pi\left(\hat{a}_{1}\right)$. Por uma conta simples, podemos verificar que $A C=B$. Portanto $z_{1}=\hat{\sigma} \hat{a}_{1} \in \hat{\sigma}$ Quat $_{4}$.

Seja uma partição $X$ de $\llbracket n+1 \rrbracket$. Além disso, lembre que definimos $\operatorname{Diag}_{n+1}^{+}=\operatorname{Diag}_{n+1} \cap \mathrm{SO}_{n+1}$.

Definição 5.2.4 O subgrupo $H_{\text {Diag, } X} \leq \operatorname{Diag}_{n+1}^{+}$de indice $2^{|X|-1}$, onde $H_{\text {Diag, } X}$ é o conjunto das matrizes $E \in \operatorname{Diag}_{n+1}^{+}$tais que, se $A=\left\{i_{1}, \ldots, i_{k}\right\} \in X$, então o produto $E_{i_{1} i_{1}} \ldots E_{i_{k} i_{k}}=1$.

Exemplo 5.2.9 Sejam $n=4$ e $X=\{\{1,3\},\{2,4,5\}\}$. Seja a matriz $E \in$

$\operatorname{Diag}_{5}^{+}$dada por

$$
E=\left(\begin{array}{ccccc}
1 & 0 & 0 & 0 & 0 \\
0 & -1 & 0 & 0 & 0 \\
0 & 0 & 1 & 0 & 0 \\
0 & 0 & 0 & -1 & 0 \\
0 & 0 & 0 & 0 & 1
\end{array}\right)
$$

Como $E_{2,2} E_{4,4} E_{5,5}=(-1) \cdot(-1) \cdot 1=1$ e $E_{1,1} E_{3,3}=1.1=1 \mathrm{com}$ $A=\{2,4,5\}, B=\{1,3\} \in X$, então $E \in H_{\text {Diag }, X}$.

Seja $H_{X}=\Pi^{-1}\left[H_{\text {Diag }, X}\right] \leq$ Quat $_{n+1}$, onde $\Pi:$ Quat $_{n+1} \rightarrow \operatorname{Diag}_{n+1}^{+}$é a restrição de $\Pi: \operatorname{Spin}_{n+1} \rightarrow \mathrm{SO}_{n+1}$.

Para uma permutação $\sigma \in \mathrm{S}_{n+1}$, considere a partição $X_{\sigma}$ de $\llbracket n+1 \rrbracket$ em ciclos de $\sigma$. Seja $H_{\sigma}=H_{X_{\sigma}} \leq$ Quat $_{n+1}$. Temos que $\left|H_{\sigma}\right|=2^{n+2-c}$, onde c é o número de ciclos de $\sigma$.

Exemplo 5.2.10 Seja $\sigma=(15)(234) \in \mathrm{S}_{5}$.

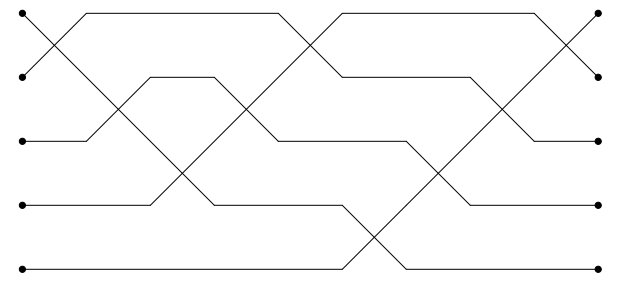

Figura 5.3: Diagrama de $\sigma=a_{1} a_{2} a_{3} a_{2} a_{1} a_{4} a_{3} a_{2} a_{1} \in \mathrm{S}_{5}$ 
Assim, $X_{\sigma}=\{\{1,5\},\{2,3,4\}\}$. Além disso, $\left|H_{\sigma}\right|=2^{4+2-2}=16$.

Por uma conta simples, podemos ver que o subgrupo $H_{\text {Diag, } X_{\sigma}}$ é gerado por

$\operatorname{diag}(-1,1,1,1,-1), \operatorname{diag}(1,-1,-1,1,1), \operatorname{diag}(1,1,-1,-1,1) \in \operatorname{Diag}_{5}^{+}$.

Levantando para $H_{\sigma}$, temos os geradores

$$
\begin{gathered}
\hat{a}_{1} \hat{a}_{2} \hat{a}_{3} \hat{a}_{4}, \hat{a}_{2}, \hat{a}_{3} \in \mathrm{Quat}_{5}, \text { daí } \\
H_{\sigma}=\left\{ \pm 1, \pm \hat{a}_{2}, \pm \hat{a}_{3}, \pm \hat{a}_{2} \hat{a}_{3}, \pm \hat{a}_{1} \hat{a}_{4}, \pm \hat{a}_{1} \hat{a}_{2} \hat{a}_{4}, \pm \hat{a}_{1} \hat{a}_{3} \hat{a}_{4}, \pm \hat{a}_{1} \hat{a}_{2} \hat{a}_{3} \hat{a}_{4}\right\}
\end{gathered}
$$

Note que $\left|H_{\sigma}\right|=16$, como esperado.

Vamos ver o resultado que associa a parte real ao número de ciclos.

Lema 5.2.2 Seja $\sigma \in \mathrm{S}_{n+1}$, escrita como um produto de ciclos disjuntos, tal que c é o número de ciclos. Escolha $z_{0} \in$ ó Quat $_{n+1}$, tal que $\mathfrak{R}\left(z_{0}\right)>0$. Seja $q \in$ Quat $_{n+1}$, temos que

$$
\left|\mathfrak{R}\left(q z_{0}\right)\right|=\left|\mathfrak{R}\left(z_{0} q\right)\right|= \begin{cases}2^{-\frac{(n+1-c)}{2}}, & q \in H_{\sigma}, \\ 0, & q \notin H_{\sigma} .\end{cases}
$$

Existem $2^{n+1-c}$ valores de $q \in$ Quat $_{n+1}$, tais que $\mathfrak{R}\left(q z_{0}\right)>0$ (da mesma forma, para $\left.\mathfrak{R}\left(z_{0} q\right)\right)$. Além disso, se podemos expandir na base canônica como $z_{0}=\sum_{p \in \mathrm{HQuat}_{n+1}} c_{p} p$, então $c_{p} \neq 0$, se e somente se, $p \in H_{\sigma}$.

Prova.

Seja $\sigma \in \mathrm{S}_{n+1}$ escrita como um produto de $c$ ciclos disjuntos. Sejam $z \in \dot{\sigma}$ Quat $_{n+1}$ e $Q=\Pi(z) \in \mathrm{SO}_{n+1}$, considere $Q_{i}$ a submatriz de dimensão $k_{i}$ associada a $c_{i}$, para $i=1, \ldots, c$ onde cada $c_{i}$ é um ciclo de $\sigma$ e $k_{1}+\cdots+k_{c}=$ $n+1$.

Se $\operatorname{det}\left(Q_{i}\right)=-1$ para algum $i$, então o polinômio característico de $Q_{i}$ é $p_{i}(\lambda)=(-\lambda)^{k_{i}}-1$, daí -1 é um autovalor de $Q_{i}$, portanto é também de $Q$. Pelo lema anterior, se -1 é autovalor de $Q=\Pi(z)$ então $\mathfrak{R}(z)=0$.

Assim se $\operatorname{det}\left(Q_{i}\right)=-1$ para algum $i$, então $\mathfrak{R}(z)=0$.

$\operatorname{Se} \operatorname{det}\left(Q_{i}\right)=1$ para todo $i$, vamos aplicar os resultados anteriores. Como $z \in \tilde{\mathrm{B}}_{n+1}^{+}$, então para cada ciclo de $\sigma$, a matriz $Q_{i}$ é um ciclo par ou ímpar cuja parte real, como vimos anteriormente, é dada por $\pm 2^{\frac{-k_{i}+1}{2}}$. Como temos $c$ ciclos disjuntos, então

$$
\mathfrak{R}(z)= \pm 2^{\frac{-k_{1}+1}{2}} \ldots 2^{\frac{-k_{c}+1}{2}}=2^{\frac{-k_{1} \cdots-k_{c}+c}{2}}= \pm 2^{\frac{-(n+1)+c}{2}}= \pm 2^{\frac{-(n+1-c)}{2}} \neq 0 .
$$


Portanto, como queremos $z_{0} \in$ ó Quat $_{n+1}$ tal que $\mathfrak{R}\left(z_{0}\right)>0$, temos que $\mathfrak{R}\left(z_{0}\right)=2^{\frac{-(n+1-c)}{2}}$ e $\operatorname{det}\left(Q_{i}\right)=1$ para todo $i$.

Afirmamos que $\mathfrak{R}\left(z_{0} q\right) \neq 0$ se, e somente se, $q \in H_{\sigma}$. De fato, se $q \in H_{\sigma}$, então os $\operatorname{det}\left(Q_{i}\right)$ ficam inalterados. Reciprocamente se $q \notin H_{\sigma}$ então algum $\operatorname{det}\left(Q_{i}\right)$ troca de sinal.

Temos então que $\mathfrak{R}\left(z_{0} q\right)= \pm \mathfrak{R}\left(z_{0}\right)= \pm 2^{\frac{-(n+1-c)}{2}}$. Aplicando o mesmo raciocínio a $q z_{0}$ temos $\mathfrak{R}\left(q z_{0}\right)= \pm 2^{\frac{-(n+1-c)}{2}}$, se $q \in H_{\sigma}$ e $\mathfrak{R}\left(q z_{0}\right)=0$, se $q \notin H_{\sigma}$.

Como $z_{0} \in$ ó Quat ${ }_{n+1} \subset \tilde{\mathrm{B}}_{n+1}^{+}$, podemos escrever

$$
z_{0}=\sum_{p \in \text { HQuat }_{n+1}} c_{p} p, \quad \text { onde } c_{p} \in \mathbb{R}
$$

Seja $X^{+} \subset$ HQuat $_{n+1}$ o conjunto de elementos cujo coeficiente na decomposição de $z_{0}$ é não nulo. Seja $X^{-} \subset$ HQuat $_{n+1}$ o conjunto das negações de $X^{+}$. Afirmamos que $H_{\sigma}=X^{+} \cup X^{-}$

Se $q \in$ Quat $_{n+1}$, então

$$
z_{0} q=\sum_{p \in X^{+}} c_{p}(p q)
$$

portanto, a parte real corresponde a $p=q^{-1}$. Ou seja, $\mathfrak{R}\left(z_{0} q\right) \neq 0$ se e somente se, $p=q^{-1} \in X^{+} \cup X^{-}$. Entretanto, sabemos que $\mathfrak{R}\left(z_{0} q\right) \neq 0$ se e somente se, $q \in H_{\sigma}$. Assim $H_{\sigma}=X^{+} \cup X^{-}$, como afirmado.

Por fim, vamos ver que existem $2^{n+1-c}$ valores de $q \in$ Quat $_{n+1}$, tais que $\mathfrak{R}\left(q z_{0}\right)>0$ (similarmente para $\mathfrak{R}\left(z_{0} q\right)>0$ ).

Como $\mathfrak{R}\left(z_{0} q\right) \neq 0$ somente se $q \in H_{\sigma}$, então temos $\left|H_{\sigma}\right|=2^{n+2-c}$ possibilidades. Sabemos que se $q \in H_{\sigma}$ então $-q \in H_{\sigma}$. Logo se $\mathfrak{R}\left(z_{0} q\right)>0$ então $\mathfrak{R}\left(z_{0}(-q)\right)<0$. Portanto, existem $\frac{2^{n+2-c}}{2}=2^{n+1-c}$ valores de $q \in$ Quat $_{n+1}$ tais que $\mathfrak{R}\left(z_{0} q\right)>0$.

Pelo mesmo raciocínio, temos o resultado para $\mathfrak{R}\left(q z_{0}\right)$.

Exemplo 5.2.11 Seja a permutação $\sigma=(13)(24)=a_{2} a_{1} a_{3} a_{2} \in \mathrm{S}_{4}$.

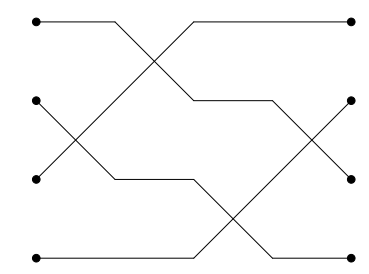

Figura 5.4: Diagrama de $\sigma=a_{2} a_{1} a_{3} a_{2} \in \mathrm{S}_{4}$ 
Então, fazendo uso das relações conhecidas para $a_{i}$, temos

$$
\hat{\sigma}=\frac{\hat{a}_{1}+\hat{a}_{2}+\hat{a}_{3}-\hat{a}_{1} \hat{a}_{2} \hat{a}_{3}}{2} .
$$

Além disso, $H_{\mathrm{Diag}, X_{\sigma}}$ é gerado por

$$
\operatorname{diag}(1,-1,1,-1), \operatorname{diag}(-1,1,-1,1) \in \operatorname{Diag}_{4}^{+} .
$$

Portanto, $H_{\sigma}$ é gerado por

$$
\begin{gathered}
\hat{a}_{1} \hat{a}_{2}, \hat{a}_{2} \hat{a}_{3} \in \mathrm{Quat}_{4}, \text { daí } \\
H_{\sigma}=\left\{ \pm 1, \pm \hat{a}_{1} \hat{a}_{2}, \pm \hat{a}_{1} \hat{a}_{3}, \pm \hat{a}_{2} \hat{a}_{3}\right\} .
\end{gathered}
$$

Agora, vamos escolher $q_{0}=-\hat{a}_{3} \in$ Quat $_{4}$. Temos que,

$$
z_{0}=-\hat{a}_{3} \dot{\sigma}=\frac{1-\hat{a}_{1} \hat{a}_{3}+\hat{a}_{2} \hat{a}_{3}+\hat{a}_{1} \hat{a}_{2}}{2} .
$$

Logo $\mathfrak{R}\left(z_{0}\right)=\frac{1}{2}>0$.

Podemos ver que os termos de $z_{0}$ coincidem com os elementos de $H_{\sigma}$. Considere $q=\hat{a}_{2} \hat{a}_{3} \in H_{\sigma}$, então

$$
\begin{aligned}
& q z_{0}=\hat{a}_{2} \hat{a}_{3} z_{0}=\frac{-1+\hat{a}_{1} \hat{a}_{2}-\hat{a}_{1} \hat{a}_{3}+\hat{a}_{2} \hat{a}_{3}}{2}, \\
& z_{0} q=z_{0} \hat{a}_{2} \hat{a}_{3}=\frac{-1-\hat{a}_{1} \hat{a}_{2}-\hat{a}_{1} \hat{a}_{3}+\hat{a}_{2} \hat{a}_{3}}{2} .
\end{aligned}
$$

Daí, $\left|\mathfrak{R}\left(q z_{0}\right)\right|=\left|\mathfrak{R}\left(z_{0} q\right)\right|=\frac{1}{2}=2^{\frac{-(n+1-c)}{2}}$, como esperávamos.

Por fim, tomemos $q=\hat{a}_{1} \notin H_{\sigma}$, então

$$
\begin{aligned}
z_{0} q= & \frac{\hat{a}_{1}+\hat{a}_{3}+\hat{a}_{2} \hat{a}_{3} \hat{a}_{1}+\hat{a}_{2}}{2}, \\
q z_{0} & =\frac{\hat{a}_{1}+\hat{a}_{3}+\hat{a}_{1} \hat{a}_{2} \hat{a}_{3}-\hat{a}_{2}}{2} .
\end{aligned}
$$

Daí, $\mathfrak{R}\left(z_{0} q\right)=\mathfrak{R}\left(q z_{0}\right)=0$ como previsto, novamente.

Exemplo 5.2.12 Seja $\sigma=(15)(234)=a_{1} a_{2} a_{3} a_{2} a_{1} a_{4} a_{3} a_{2} a_{1} \in \mathrm{S}_{5}$, como no exemplo 5.2.9. Temos que

$$
\dot{\sigma}=\frac{-\hat{a}_{1}-\hat{a}_{1} \hat{a}_{2}+\hat{a}_{1} \hat{a}_{3}-\hat{a}_{1} \hat{a}_{2} \hat{a}_{3}-\hat{a}_{4}+\hat{a}_{2} \hat{a}_{4}-\hat{a}_{3} \hat{a}_{4}-\hat{a}_{2} \hat{a}_{3} \hat{a}_{4}}{2 \sqrt{2}} .
$$

Vamos escolher um elemento $q \in$ Quat $_{5}$, por exemplo $q=\hat{a}_{1}$. Dessa 
forma,

$$
z_{0}=\hat{a}_{1} \dot{\sigma}=\frac{1+\hat{a}_{2}-\hat{a}_{3}+\hat{a}_{2} \hat{a}_{3}-\hat{a}_{1} \hat{a}_{4}+\hat{a}_{1} \hat{a}_{2} \hat{a}_{4}-\hat{a}_{1} \hat{a}_{3} \hat{a}_{4}-\hat{a}_{1} \hat{a}_{2} \hat{a}_{3} \hat{a}_{4}}{2 \sqrt{2}},
$$

logo $\mathfrak{R}\left(z_{0}\right)=\frac{1}{2 \sqrt{2}}=2^{\frac{-(n+1-c)}{2}}$.

Note que os coeficientes não nulos de $z_{0}$ coincidem com os elementos de $H_{\sigma}$ que, como vimos no exemplo anterior, implica no resultado do lema. 


\section{Sinais}

No decorrer do processo de escrita deste trabalho, surgiram questionamentos sobre a disposição dos sinais em $\dot{\sigma} \in \tilde{\mathrm{B}}_{n+1}^{+}$.

Com os resultados vistos no capítulo anterior, não podemos garantir nada sobre o sinal da parte real. Com isso, surge a questão: Quando é possível obter alguma informação sobre o sinal da parte real? Além disso, podemos observar algo sobre o comportamento dos sinais dos termos de $\sigma \in \tilde{\mathrm{B}}_{n+1}^{+}$?

Neste capítulo, faremos algumas afirmações e observações quanto a distribuição de sinais em $\sigma \in \tilde{\mathrm{B}}_{n+1}^{+}$.

\section{1}

\section{Parte Real}

Sejam $\sigma \in \mathrm{S}_{n+1}$ e $\sigma \in \tilde{\mathrm{B}}_{n+1}^{+}$. Nesta seção, vamos observar o comportamento do sinal de $\mathfrak{R}(\dot{\sigma})$, em algumas situações específicas.

Do lema 5.2.2, podemos tirar a seguinte conclusão sobre a parte real de permutações com apenas 1 ciclo.

Afirmação 6.1.1 Seja $\sigma \in \mathrm{S}_{n+1}$ tal que, o número de ciclos de $\sigma, c(\sigma)=1$. Então $\mathfrak{R}(\tilde{\sigma}) \neq 0$.

Prova.

Pelo lema 5.2.2, tomando $z_{0} \in \sigma$ Quat $_{n+1}$ tal que $\mathfrak{R}\left(z_{0}\right)>0$, temos que $\mathfrak{R}\left(q z_{0}\right)= \pm 2^{-\frac{(n+1-c)}{2}}$ se $q \in H_{\sigma}$ e $\mathfrak{R}\left(q z_{0}\right)=0$, caso contrário.

Da seção 5.2 , sabemos que $\left|H_{\sigma}\right|=2^{n+1}$. Além disso, sabemos que a cardinalidade de Quat $_{n+1}$ é também $2^{n+1}$. Portanto, se $q \in$ Quat $_{n+1}$, então $q \in H_{\sigma}$. Ou seja, para todo $q \in$ Quat $_{n+1}$, temos $\mathfrak{R}\left(q z_{0}\right) \neq 0$.

Como $\mathfrak{R}\left(z_{0}\right)>0$, então $\mathfrak{R}(\dot{\sigma}) \neq 0$.

Exemplo 6.1.1 Seja $\sigma=a_{2} a_{1} a_{3} a_{2} a_{3}=(1423) \in \mathrm{S}_{4}$. Então

$$
\hat{\sigma}_{1}=\frac{1}{2 \sqrt{2}}\left(-1+\hat{a}_{1}+\hat{a}_{2}+\hat{a}_{3}+\hat{a}_{1} \hat{a}_{2}+\hat{a}_{1} \hat{a}_{3}+\hat{a}_{2} \hat{a}_{3}-\hat{a}_{1} \hat{a}_{2} \hat{a}_{3}\right) .
$$




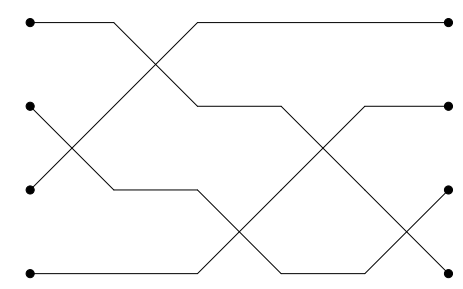

Figura 6.1: Diagrama de $\sigma=a_{2} a_{1} a_{3} a_{2} a_{3} \in \mathrm{S}_{4}$

Agora, vamos pensar em permutações $\sigma \in \mathrm{S}_{n+1}$, tais que a palavra reduzida não possua geradores $a_{i}$ repetidos.

Afirmação 6.1.2 Seja $\sigma \in \mathrm{S}_{n+1}$, tal que $\sigma=a_{i_{1}} \ldots a_{i_{l}}$ é uma palavra reduzida, onde, para todo $r, s \in \llbracket l \rrbracket$ temos $a_{i_{r}} \neq a_{i_{s}}$. Então, $\mathfrak{R}(\hat{\sigma})>0$.

Prova.

Lembre que $\dot{a}_{i}=\frac{1+\hat{a}_{i}}{\sqrt{2}}$.

Dessa forma, temos que $\hat{\sigma}=\dot{a}_{i_{1}} \ldots \dot{a}_{i_{l}}=\frac{1}{\sqrt{2}^{l}}\left(1+\hat{a}_{i_{1}}\right) \ldots\left(1+\hat{a}_{i_{l}}\right)$. Portanto, como todos os $\hat{a}_{i_{r}}$ com $r \in \llbracket l \rrbracket$ são distintos, então

$$
\mathfrak{R}(\dot{\sigma})=\frac{1}{(\sqrt{2})^{l}}>0 .
$$

Note que a afirmação é válida, independente da ordem dos geradores na palavra reduzida. Vamos ver alguns exemplos:

Exemplo 6.1.2 Seja $\sigma=a_{1} a_{3} a_{2}=(1342) \in \mathrm{S}_{4}$, temos

$$
\dot{\sigma}=\frac{1}{2 \sqrt{2}}\left(1+\hat{a}_{1}+\hat{a}_{2}+\hat{a}_{3}+\hat{a}_{1} \hat{a}_{2}+\hat{a}_{1} \hat{a}_{3}-\hat{a}_{2} \hat{a}_{3}-\hat{a}_{1} \hat{a}_{2} \hat{a}_{3}\right) .
$$

Portanto, $\mathfrak{R}(\dot{\sigma})=\frac{1}{2 \sqrt{2}}>0$

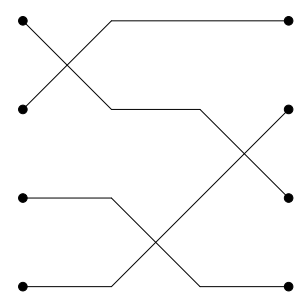

Figura 6.2: Diagrama de $\sigma=a_{1} a_{3} a_{2} \in \mathrm{S}_{4}$ 
Exemplo 6.1.3 Seja $\sigma=a_{1} a_{4} a_{2} a_{5}=(132)(546) \in \mathrm{S}_{6}$, temos

$$
\begin{gathered}
\hat{\sigma}=\frac{1}{4}\left(1+\hat{a}_{1}+\hat{a}_{2}+\hat{a}_{4}+\hat{a}_{5}+\hat{a}_{1} \hat{a}_{2}+\hat{a}_{1} \hat{a}_{4}+\hat{a}_{1} \hat{a}_{5}+\hat{a}_{2} \hat{a}_{4}+\hat{a}_{2} \hat{a}_{5}+\right. \\
\left.\hat{a}_{4} \hat{a}_{5}+\hat{a}_{1} \hat{a}_{2} \hat{a}_{4}+\hat{a}_{1} \hat{a}_{2} \hat{a}_{5}+\hat{a}_{1} \hat{a}_{4} \hat{a}_{5}+\hat{a}_{2} \hat{a}_{4} \hat{a}_{5}+\hat{a}_{1} \hat{a}_{2} \hat{a}_{4} \hat{a}_{5}\right) .
\end{gathered}
$$

Portanto, $\mathfrak{R}(\dot{\sigma})=\frac{1}{4}>0$.

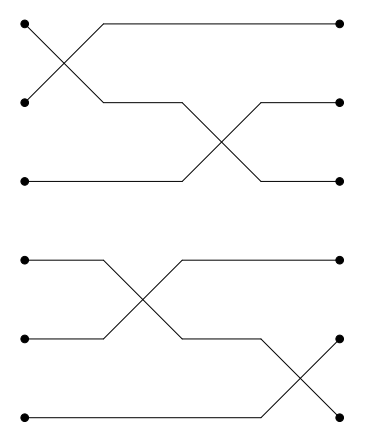

Figura 6.3: Diagrama de $\sigma=a_{1} a_{4} a_{2} a_{5} \in \mathrm{S}_{6}$

Exemplo 6.1.4 Seja $\sigma=a_{5} a_{3} a_{4} a_{2} a_{1}=(123564) \in \mathrm{S}_{6}$, temos

$$
\begin{aligned}
\hat{\sigma}=\frac{1}{4 \sqrt{2}} & \left(1+\hat{a}_{1}+\hat{a}_{2}+\hat{a}_{3}+\hat{a}_{4}+\hat{a}_{5}-\hat{a}_{1} \hat{a}_{2}+\hat{a}_{1} \hat{a}_{3}+\hat{a}_{1} \hat{a}_{4}+\hat{a}_{1} \hat{a}_{5}-\hat{a}_{2} \hat{a}_{3}+\hat{a}_{2} \hat{a}_{4}+\hat{a}_{2} \hat{a}_{5}+\right. \\
& \hat{a}_{3} \hat{a}_{4}+\hat{a}_{3} \hat{a}_{5}-\hat{a}_{4} \hat{a}_{5}-\hat{a}_{1} \hat{a}_{2} \hat{a}_{3}+\hat{a}_{1} \hat{a}_{2} \hat{a}_{4}+\hat{a}_{1} \hat{a}_{2} \hat{a}_{5}+\hat{a}_{1} \hat{a}_{3} \hat{a}_{4}+\hat{a}_{1} \hat{a}_{3} \hat{a}_{5}-\hat{a}_{1} \hat{a}_{4} \hat{a}_{5}+ \\
& \hat{a}_{2} \hat{a}_{3} \hat{a}_{4}-\hat{a}_{2} \hat{a}_{3} \hat{a}_{4}-\hat{a}_{2} \hat{a}_{3} \hat{a}_{5}-\hat{a}_{3} \hat{a}_{4} \hat{a}_{5}+\hat{a}_{1} \hat{a}_{2} \hat{a}_{3} \hat{a}_{4}+\hat{a}_{1} \hat{a}_{2} \hat{a}_{4} \hat{a}_{5}+\hat{a}_{1} \hat{a}_{2} \hat{a}_{3} \hat{a}_{5}- \\
& \left.\hat{a}_{1} \hat{a}_{3} \hat{a}_{4} \hat{a}_{5}+\hat{a}_{2} \hat{a}_{3} \hat{a}_{4} \hat{a}_{5}-\hat{a}_{1} \hat{a}_{2} \hat{a}_{3} \hat{a}_{4} \hat{a}_{5}\right) .
\end{aligned}
$$

Portanto, $\mathfrak{R}(\dot{\sigma})=\frac{1}{4 \sqrt{2}}>0$.

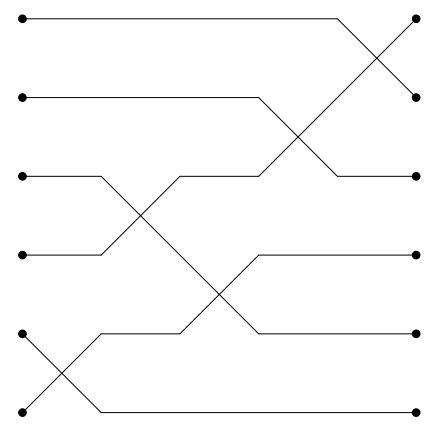

Figura 6.4: Diagrama de $\sigma=a_{5} a_{3} a_{4} a_{2} a_{1} \in \mathrm{S}_{6}$ 


\section{2}

\section{Distribuição de Sinais}

Seja $\sigma \in \mathrm{S}_{n+1}$. Podemos escrever $\sigma \in \tilde{\mathrm{B}}_{n+1}^{+}$como uma combinação linear de elementos em HQuat ${ }_{n+1}$, da seguinte forma

$$
\dot{\sigma}=\frac{1}{(\sqrt{2})^{l}} \sum_{p \in \text { HQuat }_{n+1}} c_{p} p, \quad \text { onde } c_{p} \in\{-1,0,1\} .
$$

Nesta seção, vamos observar o comportamento dos sinais de cada parcela deste somatório.

Afirmação 6.2.1 Seja $\sigma \in \mathrm{S}_{n+1}$, tal que $\sigma=a_{i_{1}} \ldots a_{i_{l}}$ é uma palavra reduzida onde para todo $r \in \llbracket l \rrbracket$, temos $i_{r}<i_{r+1}$. Então,

$$
\dot{\sigma}=\frac{1}{(\sqrt{2})^{l}} \sum_{p \in \text { HQuat }_{n+1}} c_{p} p, \quad \text { onde } c_{p} \in\{0,1\} .
$$

Prova.

Lembre que $\dot{a}_{i}=\frac{1+\hat{a}_{i}}{\sqrt{2}}$.

Dessa forma, temos que $\hat{\sigma}=\dot{a}_{i_{1}} \ldots \dot{a}_{i_{l}}=\frac{1}{\sqrt{2}}\left(1+\hat{a}_{i_{1}}\right) \ldots\left(1+\hat{a}_{i_{l}}\right)$. Observe que como todos os $\hat{a}_{i_{r}}$ são distintos, não teremos nenhum termo em que apareça algum $\hat{a}_{i_{r}}^{2}=-1$. Além disso, como cada $i_{r}<i_{r+1}$, ao efetuarmos o produto cada termo será composto de $\hat{a}_{i_{r}}^{\prime} s$, onde os índices estão em ordem crescente, ou seja, não teremos nenhum caso de troca de posições do tipo $\hat{a}_{i+1} \hat{a}_{i}=-\hat{a}_{i} \hat{a}_{i+1}$.

Portanto, cada termo do somatório terá coeficiente positivo. Assim,

$$
\dot{\sigma}=\frac{1}{(\sqrt{2})^{l}} \sum_{p \in \operatorname{HQuat}_{n+1}} c_{p} p, \quad \text { onde } c_{p} \in\{0,1\} .
$$

Exemplo 6.2.1 Seja $\sigma=a_{1} a_{2} a_{3}=(1432) \in \mathrm{S}_{4}$, temos

$$
\dot{\sigma}=\frac{1}{2 \sqrt{2}}\left(1+\hat{a}_{1}+\hat{a}_{2}+\hat{a}_{3}+\hat{a}_{1} \hat{a}_{2}+\hat{a}_{1} \hat{a}_{3}+\hat{a}_{2} \hat{a}_{3}+\hat{a}_{1} \hat{a}_{2} \hat{a}_{3}\right)
$$

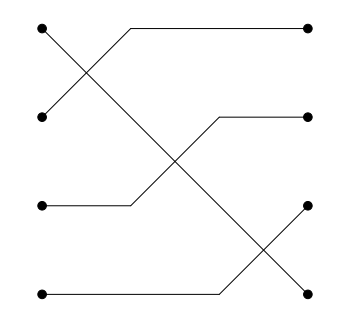

Figura 6.5: Diagrama de $\sigma=a_{1} a_{2} a_{3} \in \mathrm{S}_{4}$ 
Observe que no exemplo 6.1.2, da seção anterior, a palavra reduzida escolhida foi $\sigma=a_{1} a_{4} a_{2} a_{5} \in \mathrm{S}_{6}$. Contudo, sabemos do capítulo 2 , que $a_{i} a_{j}=a_{j} a_{i}$ se $|i-j| \neq 1$, com isso $\sigma=a_{1} a_{4} a_{2} a_{5}=a_{1} a_{2} a_{4} a_{5}$. Assim, a permutação também satisfaz as condições da afirmação 5.2.1. acima.

Exemplo 6.2.2 Seja $\sigma=a_{1} a_{4} a_{2} a_{5}=a_{1} a_{2} a_{4} a_{5}=(132)(546) \in \mathrm{S}_{6}$, temos

$$
\begin{gathered}
\dot{\sigma}=\frac{1}{4}\left(1+\hat{a}_{1}+\hat{a}_{2}+\hat{a}_{4}+\hat{a}_{5}+\hat{a}_{1} \hat{a}_{2}+\hat{a}_{1} \hat{a}_{4}+\hat{a}_{1} \hat{a}_{5}+\hat{a}_{2} \hat{a}_{4}+\hat{a}_{2} \hat{a}_{5}+\right. \\
\left.\hat{a}_{4} \hat{a}_{5}+\hat{a}_{1} \hat{a}_{2} \hat{a}_{4}+\hat{a}_{1} \hat{a}_{2} \hat{a}_{5}+\hat{a}_{1} \hat{a}_{4} \hat{a}_{5}+\hat{a}_{2} \hat{a}_{4} \hat{a}_{5}+\hat{a}_{1} \hat{a}_{2} \hat{a}_{4} \hat{a}_{5}\right) .
\end{gathered}
$$

Tendo em vista a observação e o exemplo anteriores, podemos reescrever a afirmação 6.2.1 de forma mais geral como:

Afirmação 6.2.2 Seja $\sigma \in \mathrm{S}_{n+1}$, tal que $\sigma=a_{i_{1}} \ldots a_{i_{l}}$ é uma palavra reduzida onde valem as seguintes afirmações

(i) $i_{r}<i_{r+1}$, para todo $r \in \llbracket l \rrbracket$;

(ii) se existe $r \in \llbracket l \rrbracket$ tal que $i_{r}>i_{r+1}$, então $\left|i_{r}-i_{r+1}\right| \neq 1$.

Então,

$$
\dot{\sigma}=\frac{1}{(\sqrt{2})^{l}} \sum_{p \in \operatorname{HQuat}_{n+1}} c_{p} p, \quad \text { onde } c_{p} \in\{0,1\} .
$$

Além disso, podemos também descrever exatamente como são os elementos do somatório.

Sabemos do capítulo 4, que dado $\sigma \in \mathrm{S}_{n+1}$ temos que $\left|H_{\sigma}\right|=2^{n+2-c}$. Portanto, $\tilde{\sigma} \in \tilde{\mathrm{B}}_{n+1}^{+}$descrito como

$$
\dot{\sigma}=\frac{1}{(\sqrt{2})^{l}} \sum_{p \in \text { HQuat }_{n+1}} c_{p} p, \quad \text { onde } c_{p} \in\{-1,0,1\},
$$

possui $2^{n+1-c}$ parcelas no somatório.

No caso de uma permutação como na afirmação anterior, que não possui repetições de geradores na palavra reduzida, sabemos exatamente quem são as parcelas do somatório. Basta considerarmos todas as possíveis combinações de geradores.

No exemplo 6.2.1 acima, temos a palavra $\sigma=a_{1} a_{2} a_{3}=(1432) \in \mathrm{S}_{4}$, portanto teremos $2^{3+1-1}=8$ parcelas, sendo elas:

$$
\left\{ \pm 1, \pm \hat{a}_{1}, \pm \hat{a}_{2}, \pm \hat{a}_{3}, \pm \hat{a}_{1} \hat{a}_{2}, \pm \hat{a}_{1} \hat{a}_{3}, \pm \hat{a}_{2} \hat{a}_{3}, \pm \hat{a}_{1} \hat{a}_{2} \hat{a}_{3}\right\}
$$


Contudo, sabemos pela afirmação que todos os sinais nesse caso são positivos, portanto temos

$$
\dot{\sigma}=\frac{1}{2 \sqrt{2}}\left(1+\hat{a}_{1}+\hat{a}_{2}+\hat{a}_{3}+\hat{a}_{1} \hat{a}_{2}+\hat{a}_{1} \hat{a}_{3}+\hat{a}_{2} \hat{a}_{3}+\hat{a}_{1} \hat{a}_{2} \hat{a}_{3}\right) .
$$

Para o caso de uma permutação $\sigma \in \mathrm{S}_{n+1}$, tal que a palavra reduzida não tem repetições, entretanto os geradores não estão com os índices ordenados de forma crescente, também é possível descrever a distribuição dos sinais das parcelas do somatório. Basta observarmos os geradores que estão fora de ordem.

Uma permutação $\sigma \in \mathrm{S}_{n+1}$, cuja palavra reduzida não possui geradores repetidos nos fornece um conjunto de possíveis combinações de geradores com $2^{l}$ elementos, onde $l$ é o comprimento da palavra reduzida.

Além disso, sabemos que $c_{p} \neq 0$ se, e somente se, $p \in H_{\sigma}$, portanto se $\sigma$ não possui geradores repetidos $H_{\sigma}$ possui $2^{l+1}$ elementos, daí $2^{n+1-c}=2^{l+1}$.

Sejam $x_{p}$ a sequência de índices de cada $p \in$ HQuat $_{n+1}$ e $y_{p}$ a sequência dos índices relativos a $p$ na palavra reduzida.

Exemplo 6.2.3 Seja $\sigma=a_{1} a_{4} a_{2} a_{5} \in \mathrm{S}_{6}$, como no exemplo anterior, então

$$
\dot{\sigma}=\frac{1}{(\sqrt{2})^{4}} \sum_{p \in \mathrm{HQuat}_{6}} c_{p} p, \quad \text { onde } c_{p} \in\{0,1\} .
$$

Portanto, os elementos $p \in \mathrm{HQuat}_{6}$ tais que $c_{p} \neq 0$ são os elementos de $X^{+}$. Ou seja,

$$
\begin{aligned}
p \in\left\{1, \hat{a}_{1}, \hat{a}_{2}, \hat{a}_{4}, \hat{a}_{5}, \hat{a}_{1} \hat{a}_{2}, \hat{a}_{1} \hat{a}_{4}, \hat{a}_{1} \hat{a}_{5}, \hat{a}_{2} \hat{a}_{4}, \hat{a}_{2} \hat{a}_{5}, \hat{a}_{4} \hat{a}_{5}, \hat{a}_{1} \hat{a}_{2} \hat{a}_{4},\right. \\
\\
\left.\hat{a}_{1} \hat{a}_{2} \hat{a}_{5}, \hat{a}_{1} \hat{a}_{4} \hat{a}_{5}, \hat{a}_{2} \hat{a}_{4} \hat{a}_{5}, \hat{a}_{1} \hat{a}_{2} \hat{a}_{4} \hat{a}_{5}\right\} \subset \text { HQuat }_{6} .
\end{aligned}
$$

Por fim, vamos ver quem são as sequências $x_{p}$ e $y_{p}$ dos elementos acima. Analisaremos os elementos $p_{1}=\hat{a}_{1} \hat{a}_{2} \hat{a}_{4}$ e $p_{2}=\hat{a}_{2} \hat{a}_{4} \hat{a}_{5}$, os outros seguem da mesma forma.

As sequências de indices na palavra reduzida são $y_{p_{1}}=(1,4,2)$ e $y_{p_{2}}=$ $(4,2,5)$. Já em HQuat er $_{6}$ sa $x_{p_{1}}=(1,2,4)$ e $x_{p_{2}}=(2,4,5)$.

Note que a distinção das sequências está apenas na posição dos elementos.

Agora que entendemos melhor quem são as sequências $x_{p}$ e $y_{p}$, vamos ver como elas influenciam na disposição dos sinais de $\sigma \in \tilde{\mathrm{B}}_{n+1}^{+}$.

Afirmação 6.2.3 Seja $\sigma \in \mathrm{S}_{n+1}$, tal que $\sigma=a_{i_{1}} \ldots a_{i_{l}}$ é uma palavra reduzida onde para todo $r \neq s \in \llbracket l \rrbracket$, temos $i_{r} \neq i_{s}$. Seja $I=\left\{i_{1}, \ldots, i_{l}\right\}$. Temos que 
$\dot{\sigma} \in \tilde{\mathrm{B}}_{n+1}^{+}$é dado por

$$
\dot{\sigma}=\frac{1}{(\sqrt{2})^{l}} \sum_{p \in \mathrm{HQuat}_{n+1}} c_{p} p, \quad \text { onde } c_{p} \in\{-1,0,1\} .
$$

Portanto, se $p \in H_{\sigma}$ temos:

(i) Se $x_{p}=y_{p}$, então $c_{p}=1$;

(ii) Se $x_{p} \neq y_{p}$, não apresentaremos uma fórmula, mas os exemplos indicam um algoritmo. De fato, manipulando $x_{p}$ e $y_{p}$ e levando em conta as propriedades de comutatividade e anticomutatividade dos elementos $\hat{a}_{i}$, calculamos o sinal de $c_{p}$.

Além disso, sabemos que $c_{p}=0$ se e somente se $p \notin H_{\sigma}$.

Vamos analisar novamente os exemplos 6.1.1 e 6.1.3.

Exemplo 6.2.4 Seja $\sigma=a_{1} a_{3} a_{2}=(1342) \in \mathrm{S}_{4}$. Temos que $\sigma \in \tilde{\mathrm{B}}_{n+1}^{+}$possui $2^{3}=8$ parcelas no somatório.

Como os elementos $1, \hat{a}_{1}, \hat{a}_{2}$ e $\hat{a}_{3}$ são claramente positivos, falta analisar os elementos $p_{1}=\hat{a}_{1} \hat{a}_{2}, p_{2}=\hat{a}_{1} \hat{a}_{3}, p_{3}=\hat{a}_{2} \hat{a}_{3}$ e $p_{4}=\hat{a}_{1} \hat{a}_{2} \hat{a}_{3}$.

Para $p_{1}$ e $p_{2}$ temos, $x_{p_{1}}=y_{p_{1}}$ e $x_{p_{2}}=y_{p_{2}}$, assim $c_{p_{1}}=c_{p_{2}}=1$.

Para $p_{3}$ temos $x_{p}=(2,3)$ e $y_{p}=(3,2)$. Dessa forma, $\hat{a}_{3} \hat{a}_{2}=-\hat{a}_{2} \hat{a}_{3}$, logo $c_{p_{3}}=-1$.

Por fim, para $p_{4}$ temos $x_{p_{4}}=(1,2,3)$ e $y_{p_{4}}=(1,3,2)$. Dessa forma, $\hat{a}_{1} \hat{a}_{3} \hat{a}_{2}=-\hat{a}_{1} \hat{a}_{2} \hat{a}_{3}, \operatorname{logo} c_{p_{4}}=-1$.

Portanto, temos

$$
\dot{\sigma}=\frac{1}{2 \sqrt{2}}\left(1+\hat{a}_{1}+\hat{a}_{2}+\hat{a}_{3}+\hat{a}_{1} \hat{a}_{2}+\hat{a}_{1} \hat{a}_{3}-\hat{a}_{2} \hat{a}_{3}-\hat{a}_{1} \hat{a}_{2} \hat{a}_{3}\right)
$$

Exemplo 6.2.5 Seja $\sigma=a_{5} a_{3} a_{4} a_{2} a_{1}=(123564) \in \mathrm{S}_{6}$. Temos que $\dot{\sigma} \in \tilde{\mathrm{B}}_{n+1}^{+}$ possui $2^{5}=32$ parcelas no somatório.

Consideremos o elemento $p=\hat{a}_{1} \hat{a}_{4} \hat{a}_{5} \in$ HQuat $_{n+1}$, então $x_{p}=(1,4,5)$ e $y_{p}=(5,4,1)$. Dessa forma $\hat{a}_{5} \hat{a}_{4} \hat{a}_{1}=\hat{a}_{1} \hat{a}_{5} \hat{a}_{4}=-\hat{a}_{1} \hat{a}_{4} \hat{a}_{5}$, portanto $c_{p}=-1$.

Analisando cada parcela de ó, temos

$$
\begin{aligned}
\hat{\sigma}=\frac{1}{4 \sqrt{2}} & \left(1+\hat{a}_{1}+\hat{a}_{2}+\hat{a}_{3}+\hat{a}_{4}+\hat{a}_{5}-\hat{a}_{1} \hat{a}_{2}+\hat{a}_{1} \hat{a}_{3}+\hat{a}_{1} \hat{a}_{4}+\hat{a}_{1} \hat{a}_{5}-\hat{a}_{2} \hat{a}_{3}+\hat{a}_{2} \hat{a}_{4}+\hat{a}_{2} \hat{a}_{5}+\right. \\
& \hat{a}_{3} \hat{a}_{4}+\hat{a}_{3} \hat{a}_{5}-\hat{a}_{4} \hat{a}_{5}-\hat{a}_{1} \hat{a}_{2} \hat{a}_{3}+\hat{a}_{1} \hat{a}_{2} \hat{a}_{4}+\hat{a}_{1} \hat{a}_{2} \hat{a}_{5}+\hat{a}_{1} \hat{a}_{3} \hat{a}_{4}+\hat{a}_{1} \hat{a}_{3} \hat{a}_{5}-\hat{a}_{1} \hat{a}_{4} \hat{a}_{5}+ \\
& \hat{a}_{2} \hat{a}_{3} \hat{a}_{4}-\hat{a}_{2} \hat{a}_{3} \hat{a}_{4}-\hat{a}_{2} \hat{a}_{3} \hat{a}_{5}-\hat{a}_{3} \hat{a}_{4} \hat{a}_{5}+\hat{a}_{1} \hat{a}_{2} \hat{a}_{3} \hat{a}_{4}+\hat{a}_{1} \hat{a}_{2} \hat{a}_{4} \hat{a}_{5}+\hat{a}_{1} \hat{a}_{2} \hat{a}_{3} \hat{a}_{5}- \\
& \left.\hat{a}_{1} \hat{a}_{3} \hat{a}_{4} \hat{a}_{5}+\hat{a}_{2} \hat{a}_{3} \hat{a}_{4} \hat{a}_{5}-\hat{a}_{1} \hat{a}_{2} \hat{a}_{3} \hat{a}_{4} \hat{a}_{5}\right) .
\end{aligned}
$$




\section{7}

\section{Considerações finais}

Como dito na introdução, as células de Bruhat se mostraram uma excelente ferramenta no estudo de curvas localmente convexas.

O grupo $\operatorname{Spin}_{n+1}$, que costuma causar um certo desconforto por conta da sua definição dependente de espaços de recobrimento, se torna mais acessível quando abordado matricialmente. Ao conhecermos seus geradores, conseguimos entender melhor como seus elementos se comportam.

Além disso, considerando sua estratificação de Bruhat, juntamente com o fato de que cada componente conexa de uma célula de Bruhat sem sinal, contém exatamente um elemento $z \in \tilde{\mathrm{B}}_{n+1}^{+} \subset \operatorname{Spin}_{n+1}$, que agora sabemos escrever como combinação linear de elementos de Quat ${ }_{n+1} \subset \operatorname{Spin}_{n+1}$, fomos capazes de entender ainda mais o grupo $\operatorname{Spin}_{n+1}$.

Utilizando a ordem de Bruhat, um conceito que não definimos no trabalho, na seção 1.3 enunciamos o teorema 1.3.1 que exibe um difeomorfismo entre células de Bruhat de elementos comparáveis. Relembre o enunciado.

Teorema 1 Dadas palavras reduzidas $a_{i_{1}} \cdots a_{i_{k}}<a_{i_{1}} \cdots a_{i_{k}} a_{j}$, para duas permutações consecutivas em $\mathrm{S}_{n+1}$ e dados sinais $\varepsilon_{1}, \ldots \varepsilon_{k}, \varepsilon \in\{ \pm 1\}$, defina

$$
\begin{gathered}
z_{1}=\left(\dot{a}_{i_{1}}\right)^{\varepsilon_{1}} \cdots\left(\dot{a}_{i_{k}}\right)^{\varepsilon_{k}} \\
z_{0}=z_{1}\left(\dot{a}_{j}\right)^{\varepsilon} \in \widetilde{\mathrm{B}}_{n+1}^{+} .
\end{gathered}
$$

Dado $q \in$ Quat $_{n+1}$, o mapa

$$
\begin{aligned}
\Psi: \operatorname{Bru}_{q z_{1}} \times(0, \pi) & \rightarrow \operatorname{Bru}_{q z_{0}} \\
(z, \theta) & \mapsto z \alpha_{j}(\varepsilon \theta)
\end{aligned}
$$

é um difeomorfismo.

Nas condições do teorema, podemos enunciar dois corolários.

Corolário 1 Com $z_{1}=\left(\dot{a}_{i_{1}}\right)^{\varepsilon_{1}} \ldots\left(\dot{a}_{i_{k}}\right)^{\varepsilon_{k}}, z_{0}=z_{1}\left(\dot{a}_{j}\right)^{\varepsilon} \in \tilde{\mathrm{B}}_{n+1}^{+}$e $q \in$ Quat $_{n+1}$, temos a inclusão $\overline{\mathrm{Bru}_{q z_{1}}} \subset \overline{\mathrm{Bru}_{q z_{0}}}$. 
Corolário 2 Dado $q \in$ Quat $_{n+1}$, uma palavra reduzida $a_{i_{1}} \ldots a_{i_{k}} \in \mathrm{S}_{n+1} e$ sinais $\varepsilon_{1} \ldots \varepsilon_{k} \in\{ \pm 1\}$, o mapa

$$
\begin{aligned}
\Phi:(0, \pi)^{k} & \rightarrow \operatorname{Bru}_{q(\hat{a})_{i_{1}}^{\varepsilon_{1}} \ldots(\hat{a})_{i_{k}}^{\varepsilon_{k}}} \\
\left(\theta_{1}, \ldots, \theta_{k}\right) & \mapsto q \alpha_{i_{1}}\left(\varepsilon_{i_{1}} \theta_{i_{1}}\right) \ldots \alpha_{i_{k}}\left(\varepsilon_{i_{k}} \theta_{i_{k} ;}\right)
\end{aligned}
$$

é um difeomorfismo.

O corolário 2, nos diz que a célula de Bruhat de um elemento do tipo $q(a ́ a) \dot{i}_{1}^{\varepsilon_{1}} \ldots\left(a^{\prime}\right)_{i_{k}}^{\varepsilon_{k}}$, é difeomorfa a $\mathbb{R}^{k}$. Asssim, temos parametrizações para as células de Bruhat com sinal do grupo $\operatorname{Spin}_{n+1}$.

Tal parametrização, torna possível a melhor compreensão das células que compõe a decomposição de Bruhat do grupo $\operatorname{Spin}_{n+1}$, além de ser muito útil na demonstração de teoremas que interligam células de Bruhat e curvas localmente convexas.

Em [5], outros resultados como o teorema 1.3.2 da seção 1.3, foram demonstrados utilizando as parametrizações geradas pelos resultados anteriores, juntamente com conceitos que não abordamos neste trabalho.

Com a ajuda da álgebra, que está a nosso favor por conta da estratificação de Bruhat, fomos capazes de extrair mais informações sobre os elementos de $\operatorname{Spin}_{n+1}$. Nos últimos capítulos tiramos algumas conclusões sobre a parte real de alguns elementos específicos, entretanto acreditamos ser possível entender ainda mais, o que influencia o comportamento de um elemento.

Algumas questões ainda em aberto, que podem ser temas de trabalhos futuros, são por exemplo:

1. Quando a parte real de um elemento $z \in \tilde{\mathrm{B}}_{n+1}^{+}$é diferente de zero?

2. Como é a distribuição dos sinais dos elementos $z \in \tilde{\mathrm{B}}_{n+1}^{+}$, quando escritos como combinação linear de elementos em Quat $_{n+1}$ ?

3. Quais condições algébricas influenciam na forma como os elementos de $\tilde{\mathrm{B}}_{n+1}^{+}$se apresentam? 


\section{Referências bibliográficas}

[1] ALVES, E.; SALDANHA, N. C.. On the homotopy type of intersections of two real bruhat cells. arXiv preprint arXiv:2012.11651, 2020.

[2] ATIYAH, M. F.; BOTT, R. ; SHAPIRO, A.. Clifford modules. Topology, 3:3-38, 1964.

[3] GARCIA, A.; LEQUAIN, Y.. Elementos de álgebra. Instituto de Matemática Pura e Aplicada, 2006.

[4] GORODSKI, C.. Notes on smooth manifolds. Technical report, 2013.

[5] GOULART, V.; SALDANHA, N. C.. Locally convex curves and the bruhat stratification of the spin group. Israel Journal of Mathematics, 2021 (to appear). Available at arXiv:1904.04799, 2019.

[6] HUMPHREYS, J. E.. Reflection groups and Coxeter groups, volumen 29. Cambridge university press, 1990.

[7] LEE, J. M.. Smooth manifolds. In: INTRODUCTION TO SMOOTH MANIFOLDS, p. 1-31. Springer, 2013.

[8] LIMA, E. L.. Grupo fundamental e espaços de recobrimento. Instituto de Matemática Pura e Aplicada do CN Pq., 1977.

[9] MASON, J. C.; HANDSCOMB, D. C.. Chebyshev polynomials. CRC press, 2002.

[10] SERRE, J.-P.. Lie algebras and Lie groups: 1964 lectures given at Harvard University. Springer, 2009.

[11] STILLWELL, J.. Naive lie theory. Springer Science \& Business Media, 2008.

[12] VAZ JR, J.; DA ROCHA JR, R.. An introduction to Clifford algebras and spinors. Oxford University Press, 2016. 
A

\section{Apêndice: Exemplos}

Aqui apresentamos uma lista de exemplos de $\sigma \in \tilde{\mathrm{B}}_{n+1}^{+}$, onde $\sigma \in \mathrm{S}_{n+1}$.

\section{A.1}

\section{Permutações Com 1 Ciclo}

1. $\sigma_{1}=a_{2} a_{1} a_{3} a_{2} a_{3}=a_{2} a_{1} a_{2} a_{3} a_{2}=(1423) \in \mathrm{S}_{4}$

$\hat{\sigma}_{1}=\frac{1}{2 \sqrt{2}}\left(-1+\hat{a}_{1}+\hat{a}_{2}+\hat{a}_{3}+\hat{a}_{1} \hat{a}_{2}+\hat{a}_{1} \hat{a}_{3}+\hat{a}_{2} \hat{a}_{3}-\hat{a}_{1} \hat{a}_{2} \hat{a}_{3}\right)$

2. $\sigma_{2}=a_{1} a_{3} a_{2}=(1342) \in \mathrm{S}_{4}$

$\hat{\sigma}_{2}=\frac{1}{2 \sqrt{2}}\left(1+\hat{a}_{1}+\hat{a}_{2}+\hat{a}_{3}+\hat{a}_{1} \hat{a}_{2}+\hat{a}_{1} \hat{a}_{3}-\hat{a}_{2} \hat{a}_{3}-\hat{a}_{1} \hat{a}_{2} \hat{a}_{3}\right)$

3. $\sigma_{3}=a_{1} a_{2} a_{3}=(1432) \in \mathrm{S}_{4}$

$\hat{\sigma}_{3}=\frac{1}{2 \sqrt{2}}\left(1+\hat{a}_{1}+\hat{a}_{2}+\hat{a}_{3}+\hat{a}_{1} \hat{a}_{2}+\hat{a}_{1} \hat{a}_{3}+\hat{a}_{2} \hat{a}_{3}+\hat{a}_{1} \hat{a}_{2} \hat{a}_{3}\right)$

4. $\sigma_{4}=a_{5} a_{3} a_{4} a_{2} a_{1}=(123564) \in \mathrm{S}_{6}$

$\hat{\sigma}_{4}=\frac{1}{4 \sqrt{2}}=\left(1+\hat{a}_{1}+\hat{a}_{2}+\hat{a}_{3}+\hat{a}_{4}+\hat{a}_{5}-\hat{a}_{1} \hat{a}_{2}+\hat{a}_{1} \hat{a}_{3}+\hat{a}_{1} \hat{a}_{4}+\hat{a}_{1} \hat{a}_{5}-\right.$ $\hat{a}_{2} \hat{a}_{3}+\hat{a}_{2} \hat{a}_{4}+\hat{a}_{2} \hat{a}_{5}+\hat{a}_{3} \hat{a}_{4}+\hat{a}_{3} \hat{a}_{5}-\hat{a}_{4} \hat{a}_{5}-\hat{a}_{1} \hat{a}_{2} \hat{a}_{3}+\hat{a}_{1} \hat{a}_{2} \hat{a}_{4}+\hat{a}_{1} \hat{a}_{2} \hat{a}_{5}+$ $\hat{a}_{1} \hat{a}_{3} \hat{a}_{4}+\hat{a}_{1} \hat{a}_{3} \hat{a}_{5}-\hat{a}_{1} \hat{a}_{4} \hat{a}_{5}+\hat{a}_{2} \hat{a}_{3} \hat{a}_{4}-\hat{a}_{2} \hat{a}_{3} \hat{a}_{4}-\hat{a}_{2} \hat{a}_{3} \hat{a}_{5}-\hat{a}_{3} \hat{a}_{4} \hat{a}_{5}+\hat{a}_{1} \hat{a}_{2} \hat{a}_{3} \hat{a}_{4}+$ $\left.\hat{a}_{1} \hat{a}_{2} \hat{a}_{4} \hat{a}_{5}+\hat{a}_{1} \hat{a}_{2} \hat{a}_{3} \hat{a}_{5}-\hat{a}_{1} \hat{a}_{3} \hat{a}_{4} \hat{a}_{5}+\hat{a}_{2} \hat{a}_{3} \hat{a}_{4} \hat{a}_{5}-\hat{a}_{1} \hat{a}_{2} \hat{a}_{3} \hat{a}_{4} \hat{a}_{5}\right)$

5. $\sigma_{5}=a_{2} a_{3} a_{2} a_{1} a_{2}=(3241) \in \mathrm{S}_{4}$

$$
\dot{\sigma}_{5}=\frac{1}{2 \sqrt{2}}\left(-1+\hat{a}_{1}+\hat{a}_{2}+\hat{a}_{3}-\hat{a}_{1} \hat{a}_{2}-\hat{a}_{2} \hat{a}_{3}+\hat{a}_{1} \hat{a}_{3}-\hat{a}_{1} \hat{a}_{2} \hat{a}_{2}\right)
$$

\section{A. 2}

\section{Permutações Com 2 Ciclos}

1. $\sigma_{6}=a_{1} a_{3} a_{2} a_{3} a_{4} a_{5}=(16542)(3) \in \mathrm{S}_{6}$

$\hat{\sigma}_{6}=\frac{1}{4}\left(\hat{a}_{2}+\hat{a}_{3}+\hat{a}_{1} \hat{a}_{2}+\hat{a}_{1} \hat{a}_{3}+\hat{a}_{2} \hat{a}_{4}+\hat{a}_{2} \hat{a}_{5}+\hat{a}_{3} \hat{a}_{4}+\hat{a}_{3} \hat{a}_{5}+\hat{a}_{1} \hat{a}_{2} \hat{a}_{4}+\hat{a}_{1} \hat{a}_{2} \hat{a}_{5}+\right.$ $\left.\hat{a}_{1} \hat{a}_{3} \hat{a}_{4}+\hat{a}_{1} \hat{a}_{3} \hat{a}_{5}+\hat{a}_{2} \hat{a}_{4} \hat{a}_{5}+\hat{a}_{3} \hat{a}_{4} \hat{a}_{5}+\hat{a}_{1} \hat{a}_{2} \hat{a}_{4} \hat{a}_{5}+\hat{a}_{1} \hat{a}_{3} \hat{a}_{4} \hat{a}_{5}\right)$

2. $\sigma_{7}=a_{1} a_{2} a_{3} a_{2}=(142)(3) \in \mathrm{S}_{4}$

$$
\hat{\sigma}_{7}=\frac{1}{2}\left(\hat{a}_{2}+\hat{a}_{3}+\hat{a}_{1} \hat{a}_{2}+\hat{a}_{1} \hat{a}_{3}\right)
$$

3. $\sigma_{8}=a_{1} a_{2} a_{3} a_{2} a_{1} a_{4} a_{3} a_{2} a_{1}=(15)(234) \in \mathrm{S}_{5}$

$$
\hat{\sigma}_{8}=\frac{1}{2 \sqrt{2}}\left(-\hat{a}_{2}-\hat{a}_{4}-\hat{a}_{1} \hat{a}_{2}+\hat{a}_{1} \hat{a}_{3}+\hat{a}_{2} \hat{a}_{4}-\hat{a}_{2} \hat{a}_{4}-\hat{a}_{1} \hat{a}_{2} \hat{a}_{3}-\hat{a}_{2} \hat{a}_{3} \hat{a}_{4}\right)
$$


4. $\sigma_{9}=a_{2} a_{1} a_{3} a_{2}=(13)(24) \in \mathrm{S}_{4}$

$\hat{\sigma}_{9}=\frac{1}{2}\left(\hat{a}_{1}+\hat{a}_{2}+\hat{a}_{3}-\hat{a}_{1} \hat{a}_{2} \hat{a}_{3}\right)$

5. $\sigma_{10}=a_{1} a_{3} a_{2} a_{4} a_{3}=(142)(35) \in \mathrm{S}_{5}$

$\sigma_{10}=\frac{1}{2 \sqrt{2}}\left(\hat{a}_{2}+\hat{a}_{3}+\hat{a}_{4}+\hat{a}_{1} \hat{a}_{2}+\hat{a}_{1} \hat{a}_{3}+\hat{a}_{1} \hat{a}_{4}-\hat{a}_{2} \hat{a}_{3} \hat{a}_{4}-\hat{a}_{1} \hat{a}_{2} \hat{a}_{3} \hat{a}_{4}\right)$

6. $\sigma_{11}=a_{2} a_{1} a_{3} a_{2} a_{4} a_{3} a_{4}=(153)(24) \in \mathrm{S}_{5}$

$\hat{\sigma}_{11}=\frac{1}{2 \sqrt{2}}\left(-1+\hat{a}_{1} \hat{a}_{3}+\hat{a}_{1} \hat{a}_{4}+\hat{a}_{2} \hat{a}_{3}+\hat{a}_{2} \hat{a}_{4}+\hat{a}_{3} \hat{a}_{4}-\hat{a}_{1} \hat{a}_{2} \hat{a}_{3}-\hat{a}_{1} \hat{a}_{2} \hat{a}_{3} \hat{a}_{4}\right)$

7. $\sigma_{12}=a_{2} a_{1} a_{3} a_{2} a_{4}=(13)(254) \in \mathrm{S}_{5}$

$\sigma_{12}=\frac{1}{2 \sqrt{2}}\left(\hat{a}_{1}+\hat{a}_{2}+\hat{a}_{3}+\hat{a}_{1} \hat{a}_{4}+\hat{a}_{2} \hat{a}_{4}+\hat{a}_{3} \hat{a}_{4}-\hat{a}_{1} \hat{a}_{2} \hat{a}_{3}-\hat{a}_{1} \hat{a}_{2} \hat{a}_{3} \hat{a}_{4}\right)$

8. $\sigma_{13}=a_{1} a_{4} a_{2} a_{5}=(132)(546) \in \mathrm{S}_{6}$

$$
\begin{aligned}
& \hat{\sigma}_{13}=\frac{1}{4}\left(1+\hat{a}_{1}+\hat{a}_{2}+\hat{a}_{4}+\hat{a}_{5}+\hat{a}_{1} \hat{a}_{2}+\hat{a}_{1} \hat{a}_{4}+\hat{a}_{1} \hat{a}_{5}+\hat{a}_{2} \hat{a}_{4}+\hat{a}_{2} \hat{a}_{5}+\hat{a}_{4} \hat{a}_{5}+\right. \\
& \left.\hat{a}_{1} \hat{a}_{2} \hat{a}_{4}+\hat{a}_{1} \hat{a}_{2} \hat{a}_{5}+\hat{a}_{1} \hat{a}_{4} \hat{a}_{5}+\hat{a}_{2} \hat{a}_{4} \hat{a}_{5}+\hat{a}_{1} \hat{a}_{2} \hat{a}_{4} \hat{a}_{5}\right)
\end{aligned}
$$

\section{A.3}

\section{Permutações Com 3 Ciclos}

1. $\sigma_{14}=a_{1} a_{2} a_{3} a_{4} a_{3} a_{1}=(153)(2)(4) \in \mathrm{S}_{5}$ $\hat{\sigma}_{14}=\frac{1}{2}\left(\hat{a}_{1} \hat{a}_{3}+\hat{a}_{1} \hat{a}_{4}+\hat{a}_{2} \hat{a}_{3}+\hat{a}_{2} \hat{a}_{4}\right)$

2. $\sigma_{15}=a_{2} a_{1} a_{3} a_{2} a_{5}=(13)(24)(56) \in \mathrm{S}_{6}$ $\hat{\sigma}_{15}=\frac{1}{2 \sqrt{2}}\left(\hat{a}_{1}+\hat{a}_{2}+\hat{a}_{3}+\hat{a}_{1} \hat{a}_{5}+\hat{a}_{2} \hat{a}_{5}+\hat{a}_{3} \hat{a}_{5}-\hat{a}_{1} \hat{a}_{2} \hat{a}_{3}-\hat{a}_{1} \hat{a}_{2} \hat{a}_{3} \hat{a}_{4}\right)$

3. $\sigma_{16}=a_{1} a_{2} a_{1} a_{5}=(13)(45)(2) \in \mathrm{S}_{5}$ $\hat{\sigma}_{16}=\frac{1}{2}\left(\hat{a}_{1}+\hat{a}_{2}+\hat{a}_{1} \hat{a}_{5}+\hat{a}_{2} \hat{a}_{5}\right)$

4. $\sigma_{17}=a_{1} a_{2} a_{1} a_{3} a_{2} a_{1} a_{4} a_{3} a_{2} a_{1} a_{5} a_{4} a_{3} a_{2} a_{1}=(16)(25)(34) \in \mathrm{S}_{6}$ $\sigma_{17}=\frac{1}{2 \sqrt{2}}\left(1+\hat{a}_{3}-\hat{a}_{2} \hat{a}_{4}-\hat{a}_{2} \hat{a}_{3} \hat{a}_{4}-\hat{a}_{1} \hat{a}_{5}-\hat{a}_{1} \hat{a}_{3} \hat{a}_{5}+\hat{a}_{1} \hat{a}_{2} \hat{a}_{4} \hat{a}_{5}+\hat{a}_{1} \hat{a}_{2} \hat{a}_{3} \hat{a}_{4} \hat{a}_{5}\right)$

\section{A.4}

\section{Permutações Com 4 Ciclos}

1. $\sigma_{18}=a_{1} a_{2} a_{1} a_{5} a_{4} a_{5}=(13)(2)(46)(5) \in \mathrm{S}_{6}$

$\hat{\sigma}_{18}=\frac{1}{2}\left(a_{1} a_{4}+a_{1} a_{5}+a_{2} a_{4}+a_{2} a_{5}\right)$

2. $\sigma_{19}=a_{1} a_{2} a_{4} a_{3} a_{4} a_{2} a_{1}=(15)(2)(3)(4) \in \mathrm{S}_{5}$

$$
\hat{\sigma}_{19}=\frac{1}{\sqrt{2}}\left(\hat{a}_{2} \hat{a}_{4}+\hat{a}_{1} \hat{a}_{3}\right)
$$

